\title{
Model Hydrographs
}

GEOLOGICAL SURVEY WATER-SUPPLY PAPER 2005

Prepared in cooperation with State of Illinois Department of Public Works and Buildings, Division of Highways

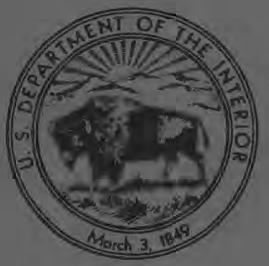




\section{Model Hydrographs}

By W. D. MITCHELL

GEOLOGICAL SURVEY WATER-SUPPLY PAPER 2005

Prepared in cooperation with State of Illinois Department of Public Works and Buildings, Division of Highways

$A$ discussion of the use of time and areal characteristics of a drainage basin to describe its flood hydrograph

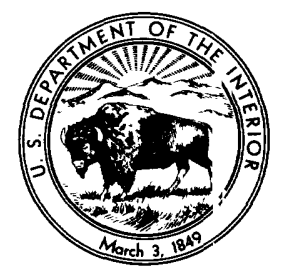




\section{UNITED STATES DEPARTMENT OF THE INTEPIOR}

ROGERS C. B. MORTON, Secretary

\section{GEOLOGICAL SURVEY}

V. E. McKelvey, Director

Library of Congress catalog-card No. 79-186800

For sale by the Superintendent of Documents, U.S. Government Printing Office Washington, D.C. 20402 - Price 45 cents (paper cover)

Stock Number 2401-2050 


\section{CONTENTS}

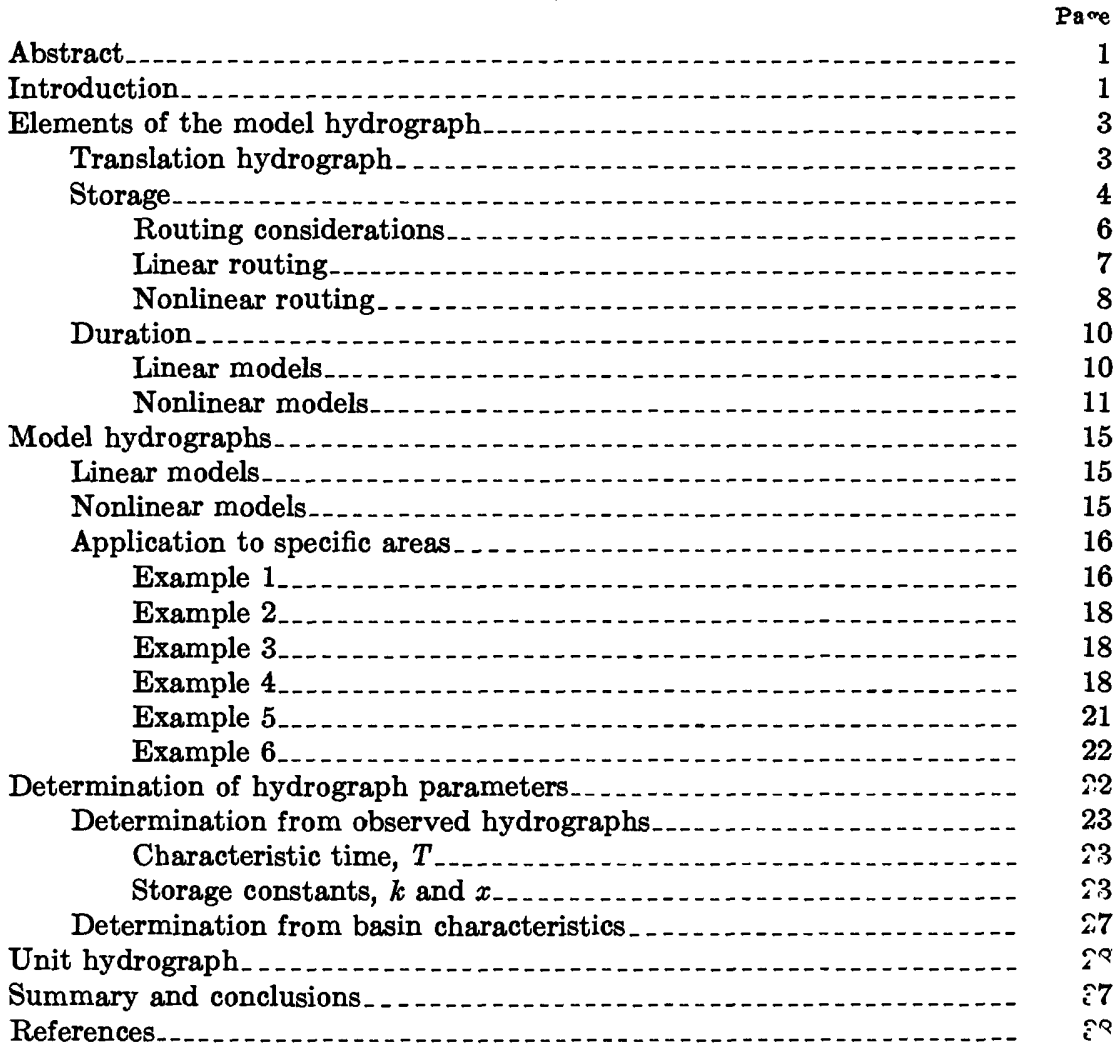

\section{ILLUSTRATIONS}

Figure

1. Translation hydrograph, instantaneous.

Page

2. Translation hydrographs, finite values of $D / T$

3-6. Hydrographs for:

3. Tributary to Second Creek at Keptown, Ill .......

4. Tributary to Kankakee River near Bourbonnais, Ill.

5. Mazon River near Coal City, Ill

6. Tributary to Vermilion River at Lowell, Ill., and for tributary to Mud Creek near Tower Hill, Ill. ... 
Figures 7-10. Graphs of:

7. Analyses for storage constants of model hydrographs. . . . . .

8. Analyses for storage constants of observed hydrographs.

9. Observed versus computed values of $T \ldots \ldots$

10. Observed versus computed values of $k \ldots \ldots$

11. Proportional graphs, resulting from routing through linear storage, $S=0.20 O$

12. Nonproportional graphs, resulting from routing through nonlinear storage, $S=10 O^{0.5}$

13. Nonproportional graphs, resulting from routing through nonlinear storage, $S=0.01 O^{2}$

14. Semilogarithmic plotting, recession limbs of grafhs from figures 11,12 , and $13 \ldots$

15. Typical recessions, previously published unit hydrographs

16. Recession hydrographs, all for Money Creek abcve Lake Bloomington, Ill., computed by various methods.......

Page

25

26

29

30

31

32

33

34

35

36

\section{TABLES}

TABle

1. Section of typical model hydrograph (linear) compulations.-

2. Transformation of translation hydrograph from instantaneous to finite values of $D / T$.

Page

3-28. Linear model hydrographs $(S=k O) ; k / T=$ :

$\begin{aligned} \text { 3. } & 0.20 \\ \text { 4. } & 0.25 \\ 6 . & 0.30 \\ 7 . & 0.40 \\ 8 . & 0.45 \\ 9 . & 0.50 \\ 10 . & 0.55 \\ 11 . & 0.60 \\ 12 . & 0.70 \\ 13 . & 0.80 \\ 14 . & 0.90 \\ 15 . & 1.00 \\ 16 . & 1.10 \\ 17 . & 1.20 \\ 18 . & 1.30 \\ 19 . & 1.40 \\ 20 . & 1.50 \\ 21 . & 1.60 \\ 22 . & 1.80 \\ 23 . & 2.00 \\ 24 . & 2.20 \\ 25 . & 2.40 \\ 26 . & 2.60 \\ 27 . & 2.80 \\ 28 . & 3.00\end{aligned}$


TABLES 29-36. Nonlinear model hydrographs $\left(S=k O^{x}\right) ; x=0.5 ; \quad$ Pere

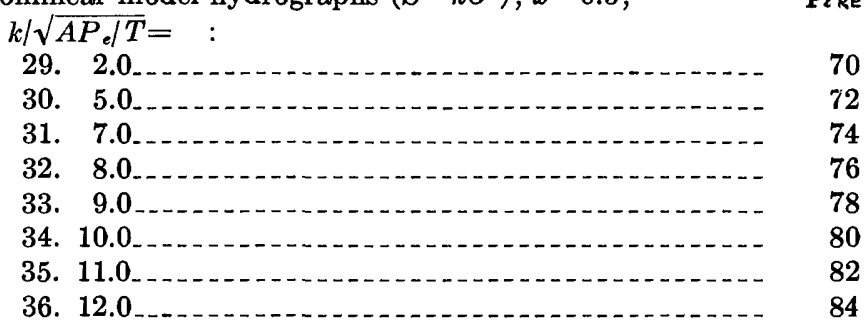

\section{SYMBOLS}

(The following symbols are listed in alphabetical order. Each symbol indicates the basic term usually represented, with no attempt to show the many and unavoidable duplicate uses.)

$A$ Drainage area (square miles).

$D$ Duration of rainfall excess (hours).

$H$ Time from beginning of rainfall excess (hours); also change in elevation between highest point on the drainage divide and channel bed at gage (feet).

I Inflow (sometimes expressed in units of cubic feet per second, but mone often in terms of the dimensionless ratio $Q T / A P_{e}$ ).

$k \quad$ A coefficient in the relation between storage and outflow (hours).

$L \quad$ Length of a drainage basin (miles).

$O$ Outflow (sometimes expressed in units of cubic feet per second, but mo*e often in terms of the dimensionless ratio $\left.Q T / A P_{e}\right)$.

$P_{e} \quad$ The amount of rainfall excess (inches).

$Q \quad$ Rate of discharge (cubic feet per second).

$S \quad$ Basin storage (cubic feet).

$T$ Base length of instantaneous translation hydrograph (hours).

$t$ Increment between successive abscissa of hydrographs (usually dimensionless).

$x \quad$ Exponent in the relation between storage and outflow (dimensionless). 


\title{
MODEL HYDROGRAPHS
}

\author{
By W. D. Mitchell
}

\section{ABSTRACT}

Model hydrographs are composed of pairs of dimensionless ratios, arrayed in tabular form, which, when modified by the appropriate values of rainfall excess and by the time and areal characteristics of the drainage basin, satisfactorily represent the flood hydrograph for the basin.

Model hydrographs are developed from a dimensionless translation hydrograph, having a time base of $T$ hours and appropriately modified for storm duration by routing through reservoir storage, $S=k O^{x}$. Models fall into two distinct classes: (1) those for which the value of $x$ is unity and which have all tl ? characteristics of true unit hydrographs and (2) those for which the value of $x$ is other than unity and to which the unit-hydrograph principles of proportionality and superposition do not apply.

Twenty-six families of linear models and eight families of nonlinear models in tabular form form the principal subject of this report. Supplemental discussions describe the development of the models and illustrate their application. Other sections of the report, supplemental to the tables, describe methods of determinir ? the hydrograph characteristics, $T, k$, and $x$, both from observed hydrograpl ? and from the physical characteristics of the drainage basin.

Five illustrative examples of use show that the models, when properly converted to incorporate actual rainfall excess and the time and areal characteristics of the drainage basins, do indeed satisfactorily represent the observed flood hydrographs for the basins.

\section{INTRODUCTION}

This report presents an array of model hydrographs and explairs their development, their use, and their relation to unit hydrographs.

A model hydrograph is a generalized expression for the distribution, with respect to time, of the surface runoff from a drainage ares. Although expressed in terms that make it applicable to many areas, it may be readily converted to explicit terms for a particular ares. Like the unit hydrograph, it is not concerned with the amount of rainfall excess that may result from a given pattern of rainfall, but only with the time distribution of the excess.

A model hydrograph is distinctive from a unit hydrograph. The latter normally is prepared for a particular drainage area and for a unit amount of rainfall excess in a particular time. These restrictions 
limit the use of the unit hydrograph to the area from which it is derived. The model hydrograph, on the other hand, is designed to apply to a wide range of sizes and types of drainage areas that have a wide range of conditions of rainfall excess. This application is facilitated by expression of the coordinates of the models in terms of dimensionless ratios.

Another important distinction between the two concepts, as will be shown subsequently, is that the unit-hydrograph principle of proportionality of ordinates limits use of the unit hydrograph to those areas for which the storage effect is linear. Many drainage areas do, indeed, appear to have linear storage - or storage so $r$ ear to linear that the differences may be disregarded. The widespread success of the customary unit-hydrograph procedures is adequate testimony to this fact. But occasionally an area is found for which storage effects are so far from linear that unit-hydrograph techniques are not applicable. For areas with marked nonlinear storage, ordinates of hydrographs are not in linear proportion to rainfall excess, and the unit-hydrograph techniques of proportionality and superposition cannot $\mathrm{k} \odot$ applied. To include these types of areas, the general principles for analysis and synthesis of hydrographs must be expanded. The term "model hydrograph" has been adopted to designate this new and bronder concept, under which the unit hydrograph becomes a special, simplified case of the model hydrograph.

The full implications of this more generalized approach to the analysis and synthesis of the hydrograph are not, as yet, completely realized. Some of the techniques which eventually may be of general use, as well as some of those which must be restricted to the special case of the unit hydrograph, have not yet been recognized. Much work remains to be done, especially with regard to the effects of nonuniform distribution of rainfall excess and the effects, for nonlinear models, of high initial inflow from antecedent rainfall. Nevertheless, significant progress has been made, and a record of that progress is the theme of this report.

The basic concepts of this report were developed over a period of several years. Materials and conclusions from other projects within the Illinois District of the Water Resources Division have been appropriated as needed. For example, the adoption of the dimensionless form for the hydrographs resulted from earlier studies (I Mitchell, 1948 and, particularly, Mitchell, 1962). Needed impetus for the completion of the project did not develop, however, until intensive analyses were undertaken in connection with a district project intended to determine floodflows from small drainage areas and prosecuted in cooperation with the Illinois Department of Public Works and Buildings, Division of Highways. As it became more apparent that the techinaues described 
in this report were well adapted to analysis of the small-basin flood data and that these projects would clearly benefit from each other, the two projects were closely coordinated. Many of the tables and most of the illustrations which appear in this report have been taken from the work done in connection with the Division of Highways cooperative project.

In the preparation of this report. three other members of the district staff have rendered invaluable assistance. George W. Curtis supervised the long and tedious manual computations for the linear models, a chore which has extended intermittently over a period of about 8 years. Terence E. Harbaugh contributed many valuable suggestiors, reviewed the manuscript with great care, made numerous tests of validity, and provided the computations for the nonlinear models. Oscar G. Lara supervised the statistical analyses and contributed suggestions which led to the systematization of the nonlinear models.

\section{ELEMENTS OF THE MODEL HYDROGRAPH}

Three principal elements enter into the concept and the use of model hydrographs. These are (1) the concept of the dimensionless, instantaneous translation hydrograph, (2) the effects of storage, ard (3) the effects of storm duration.

\section{TRANSLATION HYDROGRAPH}

To understand the translation hydrograph, consider a strang?, wonderland basin in which there is no such thing as storage. Runoff from such a basin would differ greatly from that to which we are accustomed. As soon as a rainfall excess appeared on any part of a drainage basin, it would flow immediately to the drainage outlet. ("Immediately" is an ambiguous term, but let it suffice for tre present.) The result at the outlet would be first, a quick, sharp rise to a peak greatly in excess of present natural occurrence and second, a very rapid recession to a state of no flow.

How quick, how sharp, and to what peak? These questions may $\mathrm{r}$. answered by a more deliberate consideration of translation flow, a purely hypothetical type of flow uninfluenced by storage. Let a drainage area, $A$, be divided into equal, minute parts, $d A$. Assume a magic carpet whereby rainfall excess accumulating on any $d A$ may $\mathrm{k}$. transported to the outlet in an interval of time proportional to the distance it must travel. Now assume that rainfall excess of any deptl, $P_{e}$, appears instantaneously, simultaneously, and uniformly over the entire basin, $A$. Then flow at the outlet would occur as follows: During the first instant, flow from the area immediately adjacert to the outlet;during the next instant, flow from those $d A$ 's immediately beyond the first; at any time, $H$, after appearance of rainfall excess,

$446-3750-72-2$ 
flow would be from those areas removed from the outlet by the distance corresponding to the value of $H$, and the rate of $f$ low would be proportional to the number of $d A$ 's at that particular distance. Flow would increase to a maximum for that particular value of $H$ at which the corresponding distance taps the maximum number of $d A$ 's: it would then begin to decrease and would fall to zero after that time, $T$, when the flow arrives from the most distant $d A$.

The total volume of flow, $V$, would equal the product of $A$ and $P_{e}$; if $V$ were expressed in cubic feet, with $A$ in square milos and $P_{e}$ in inches, $V=A P_{e}(5,280)^{2} / 12$, or $V=2,323,200 A P_{e}$. Since the entire flow would pass the outlet in the period of $T$ hours, the average rate of flow would be $Q_{a}=2,323,200 A P_{e} / 3,600 \quad T=645.333 A P_{e} T$ cubic feet per second.

The preceding considerations define, in a general way, the translation hydrograph for instantaneous rainfall excess. The hydrograph is for a drainage area of $A$ square miles and represents instantaneous rainfall excess of $P_{e}$ inches; it has a time base of $T$ hours and an average ordinate of $645.333 A P_{e} / T$ cubic feet per second. The precise shape of the graph is dependent upon the shape of the drainage area and upon how distances (between the $d A$ 's and the outlet) are mearured. Should distances be measured by air line? Should they be measured along principal stream channels? Should they be weighted to allow for differences in slope and roughness? Experience with many hydrographs indicates that, in general, these questions are not important. In rare instances, one or another of these considerations may become significant, but these will be dealt with at a later time, perhaps in a subsequent report. For the present, and for the large majority of drainage areas, a sufficiently accurate assumption is that the maximum ordinate of the instantaneous translation hydrograph is twice the average ordinate and occurs at time $H=T / 2$ and that the graph is two straight lines connecting this maximum ordinate to the zero ordinate at $H=O$ and at $H=T$. Subsequent manipulations of this graph are so extensive that even appreciable variations from this ideal shape become almost completely obliterated.

One further observation concerning this hydrograph is that its usefulness is greatly enhanced by reducing it to dimensionless terms, $Q T / A P_{e}$ for the ordinate and $H / T$ for the abscissa. Figure 1 presents in dimensionless form the instantaneous translation hydrograph.

\section{STORAGE}

The instantaneous translation hydrograph, as shown in figure 1, is only a starting point for further developments toward a model hydro- 


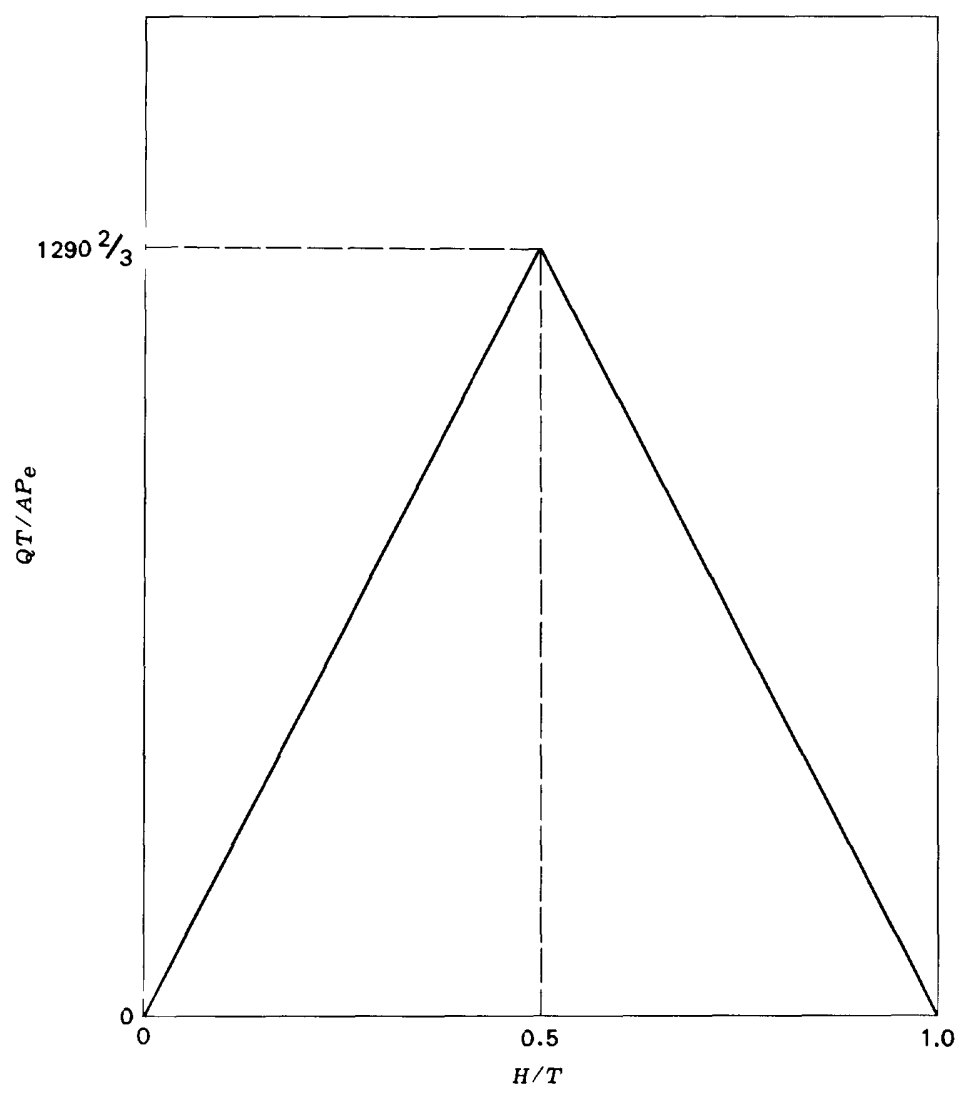

Fugure 1.-Translation hydrograph, instantaneous.

graph. For natural drainage areas, the runoff hydrograph never appears in this simple form, but is modified by at least two important factors. One of these factors is storage. (The other, duration, will be discussed later.)

The extremely diverse and complicated nature of the actual storage? facilities within a drainage basin would appear, at first glance, to preclude any successful effort toward basin-wide evaluation. But experience with many hydrographs indicates that, in general, and for the large majority of drainage areas, the effect of basin storage may be represented as a modification of the translation hydrograph by the techniques of reservoir routing, and that, as will be shown subsequently, to determine the storage relationships needed to make the modification for a particular drainage area is usually feasible. 


\section{ROUTING GONSIDERATIONS}

The basic relation between inflow, outflow, and storage is expressed by the equation of continuity, which for an incompressible fluid such as water may be written

$$
I=O+\Delta S / \Delta t
$$

that is, the inflow, $I$, is equal to the outflow, $O$, plus the change of storage, $\Delta S / \Delta t$. If the time increment, $\Delta t=t_{2}-t_{1}$, is sufficiently small, the values of $I$ and $O$ may be considered to be the average of their respective values at time $t_{1}$ and $t_{2}$, or $\left(I_{1}+I_{2}\right) / 2$ and $\left(O_{1}+O_{2}\right) / 2$. Further, $\Delta S$ may be expressed as the difference in storage volume at times $t_{1}$ and $t_{2}$, or $\Delta S=S_{2}-S_{1}$. Equation 1 may then be expressed as

$$
O_{2}=\left(2 S_{1} / \Delta t\right)-O_{1}-\left(2 S_{2} / \Delta t\right)+\left(I_{1}+I_{2}\right) \text {. }
$$

Three items of information are required by equation 2 . One of these is a starting value, or the value of $O_{1}$ at the beginning of the first increment of time, $\Delta t$. (For succeeding increments of time, $O_{1}$ is the $O_{2}$ of the preceding step.) To obtain the initial $O_{1}$, simply start the computations at a time for which the outflow is known, as at the beginning of a flood period, when the outflow generally is equal to the inflow. In many cases the initial outflow will be zero, or at least so small as to be of no practical consequence. In fact, for simplicity in the developments immediately following, initial outflow is assumed to be zero.

The second item of information is an inflow hydrograph from which to determine values of $I_{1}$ and $I_{2}$ for successive times at intervals of $\Delta t$. This information is provided by graphs such as figure 1 , the instantaneous translation hydrograph.

The third item needed for computation of $\mathrm{O}_{2}$ is a definitive expression for storage. In reservoir routing, storage is assumed to be a function of outflow and is represented by the generalized expression

$$
S=k O^{x}
$$

in which $k$ is a coefficient of proportionality, $O$ is the ontflow, and $x$ is an exponent dependent upon the relative slopes of the stage-discharge and the stage-storage relations.

A special case arises when the value of $x$ is unity. Tlen, and only then, the storage is said to be linear. Many runoff hydrographs exhibit the characteristics of linear storage. The number of exceptions, however, is so great that nonlinear storage ( $x$ other than unity) cannot be ignored. But let us first consider the simple, snecial caselinear storage, or

$$
S=k O \text {. }
$$




\section{LINEAR ROUTING}

In equation $4, S$ has the dimension of cubic feet, and $O$ has the dimension of cubic feet per second. Therefore, $k$ must have the dimension of time. Routing computations may be greatly simplified by expressing $k$ in dimensionless form, or as a ratio to $T$. Let $k / T=r$; then $S=r T O$.

In the generalized routing expression, equation $2, \Delta t$ also has the dimension of time. Here, again routing computations will be facilitated by expressing $\Delta t$ as a ratio to $T$. Let $\Delta t / T=p$. Then

$$
2 S / \Delta t=2 r T O / p T=(2 r / p) O .
$$

Equation 2 then becomes

or

$$
O_{2}=(2 r / p) O_{1}-O_{2}-(2 r / p) O_{2}+\left(I_{1}+I_{2}\right)
$$

$$
O_{2}(2 r / p+1)=O_{1}(2 r / p-1)+\left(I_{1}+I_{2}\right) .
$$

For any specific problem with linear storage, $r$ will have a fixed sF ${ }^{3-}$ cific value, such as $r=k / T=0.20$, and $p$ will have a fixed specific value, such as $p=\Delta t / T=0.02$; hence $2 r / p$ will have a specific value, such as $2 \times 0.20 / 0.02=20$, and equation 5 will assume the form

$$
O_{2}=\left(19 O_{1}+I_{1}+I_{2}\right) / 21 \text {. }
$$

Note that this simple form of the routing equation is appropriate only when storage is a linear function of outflow and that the constants in the equation will vary with the values of $k$ and $\Delta t$. For example, if $k$ becomes $0.5 T(r=0.5)$ while $\Delta t$ remains $0.02 T(p=0.02)$, then

$$
O_{2}=\left(49 O_{1}+I_{1}+I_{2}\right) / 51 \text {. }
$$

Values of $k$ used in this report cover a considerable range, but the value of $\Delta t$ has been kept the same for most linear routing computations. As pointed out above, $\Delta t$ should be small enough that the average values of $I$ and $O$ during the interval will be virtually the same as the average of their respective values at the beginning and end of the interval. For this purpose alone a time increment of $0.1 T$ might be sufficiently small; however, in order that there may be no doubt as to the accuracy of the computations and also to provide more accurate data concerning both time and magnitude of peak discharge, the value of $\Delta t$ has been taken as $0.02 T$ for most linear routing computations. (For exceptions, see footnotes to tables at end of report.) 
Table 1 presents a section of a typical linear routing computation. In this instance, the storage equation is $S=0.20 O$, or $k / T=0.20$; the routing equation assumes the specific form of equation $5 \varepsilon$. Columns 1 and 2 are a listing of the coordinates of figure 1 , the instantaneous translation hydrograph, at intervals of $\Delta t=0.02 T$. Any given line in column 3 is the sum of column 2 on that line and column 2 on the preceding line; this process provides the $I_{1}+I_{2}$ terms of the routing equation. Column 4 is the modified, or outflow, instantaneous hydrograph and is obtained by taking a value from column 4, multiplying it by 19, then adding the value of column 3 of the following line, and dividing the sum by 21 . (See equation 5a.) The quotient is entered as the value for the second line of column 4 . The remairing columns of table 1 will be discussed subsequently.

\section{NONLINEAR ROUTING}

Nonlinear routing must take account of problems that are numerous, varied, and of considerable importance. One of these is the adjustments for duration, but this problem must be reserved until the whole subject of duration is considered. Other problems concern the magnitude of flow at the beginning of rainfall excess and th a magnitude of rainfall excess. These, too, will be reserved for later discussion. The problem which deserves immediate attention is the solution of the nonlinear routing equation.

When, in equation $3, x$ assumes any value other than unity, equation 2 cannot be reduced to a simple form such as equation 5 a. Except for a few special cases, the routing equation remains so cumbersome that solutions are prohibitively tedious or are rough and unsatisfactory approximations. (This situation is improved by use of an electronic computer, but such equipment became available only near the end of this study.)

Fortunately, there are at least two special cases in which the routing equation may be solved with satisfactory accuracy and reasonable speed on desk calculators which are able to abstract square root. These cases are

and

$$
x=2.0
$$

$$
x=0.5 \text {. }
$$

The first case now appears to have limited practical ralue in that $x$ for natural drainage basins appears rarely to exceed unity. On the other hand, the second case is quite practical; several small drainage basins have been found to have a value of $x$ near 0.5 .

Results of the nonlinear routings will be described as the need arises. 


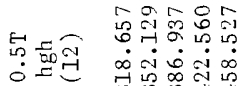

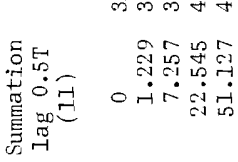

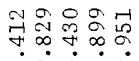

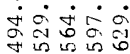

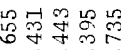

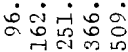

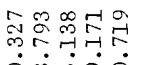

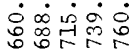

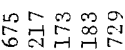 \\ क्ष \\ तीनी}

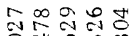

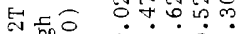

O.

옹

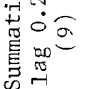

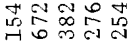

它客客客

तरतिकोल

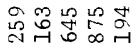

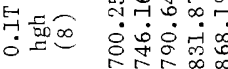

동

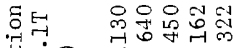

मำ

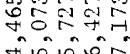

क्ष

नी मी

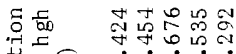

늘

吾:

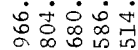

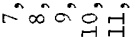

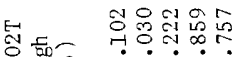

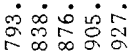

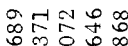

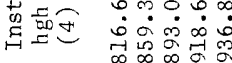

萄

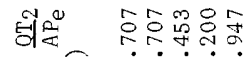

+ $\widehat{0} \dot{0} \dot{0} \dot{0}$

붕

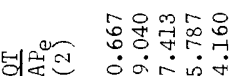

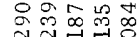
नित्जिन

푀터
Hed is a

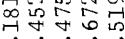

$\begin{array}{lll}\infty & \infty & \infty \\ 2 & \infty & \infty \\ \infty & \infty & \infty\end{array}$

앙N

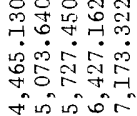

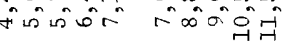

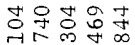

$\dot{0} \dot{0} \dot{0}$

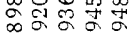

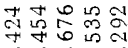

ㅎํ엉

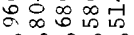

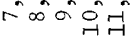

苦

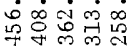

ริตึปี่

성궏ㅇㅇㅇ잉

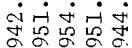

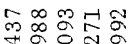

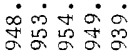

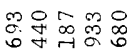

$\dot{0} \dot{0}$

7 덩해용

वतनीत

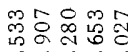

iं

(1)

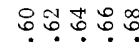

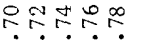

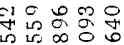

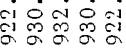

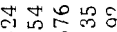

$\dot{0} \dot{0} \dot{0} \dot{0}$

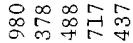

过宁宗志这

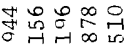

$\dot{0} \infty$ 히

जित्ञ

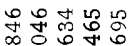

可家家审

जीजें0

요용

गิ

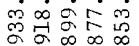

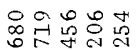

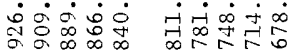

궉웜웡

ठ효영

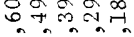

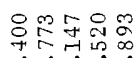

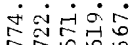

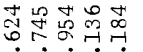

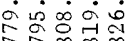

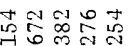

형

ततनीक

武热总员范

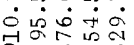

$+\infty \infty \infty$

객구

$0 \infty$ i $\infty$

4

สิ่ามี

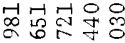

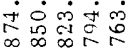

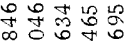

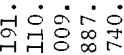

जิनेंतें

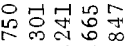

bi:

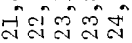

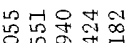

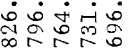

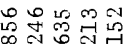

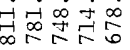

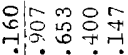

मं०ें

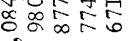

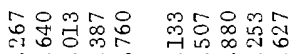

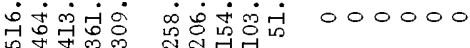

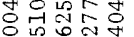

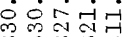

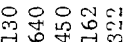

$\sin 20$

ชํำ

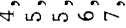

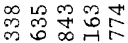

वंक्षेक्ष

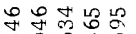

$\dot{0} \dot{0} \dot{0} \dot{0}$

न $7-18 \infty$

जึतें

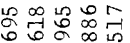

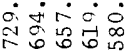

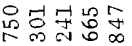

$\dot{\circ} \dot{\infty} \dot{0}$ i

员

สิสี่ง

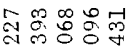

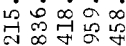

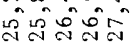

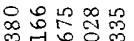

वेन्में में

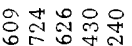

명요

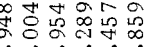

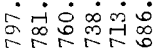

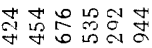

$\dot{0} \dot{0} \dot{0} \dot{0} \dot{0}$

ก⿻ำ

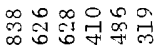

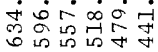

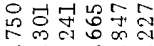

$\dot{0} \dot{\infty} \dot{0} \dot{0}$

员

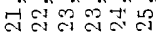

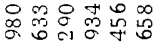

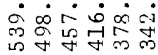

ㄱำ $200 \%$

$\dot{0} \dot{0}$

겅

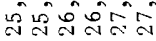

스요요

근

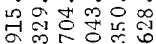

तi

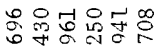

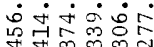

국요

में

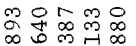

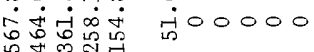

हु)

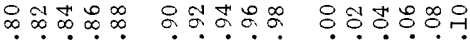




\section{DURATION}

The preceding section has shown how the instantaneous translation hydrograph may be modified by reservoir routing to protuce hydrographs such as that shown as column 4 of table 1 . Some of these begin to resemble actual, natural, hydrographs. But ther still is one important difference: duration, or the interval of time during which rainfall excess is generated.

In the development of the initial hydrograph (fig. 1), it was specified that "rainfall excess of any depth, $P_{e}$, appears instantaneously, simultaneously, and uniformly over the entire basin, A." Routing a hydrograph does not change its rainfall-excess characteristics. We must recognize, therefore, that the resulting graphs are for rainfall excess that "appears instantaneously, simultareously, and uniformly over the basin." Because these characteristics are unrealistic, our models must be adjusted to accommodate rainfall excess as it actually occurs. Adjustments for lack of simultaneous and uniform excess must await later consideration, but the adjustment for duration (finite, rather than instantaneous) may be made now.

The duration, $D$, or time interval during which rainfall excess is generated, obviously has the dimension of time and normally is expressed in hours. In keeping with the dimensionless character of the hydrographs so far developed, however, let duration be expressed by the dimensionless ratio, $D / T$.

\section{LINEAR MODELS}

For linear models, transformations for duration normally are effected, after routing for storage, by means of the summation curve, as follows: Prepare a summation curve for any available hydrograph whose $D / T$ value is $z$; list a second summation curve identical to first but lagged by a time interval equal to the desir?d duration, $D / T=y$; subtract the ordinates of the second curve from those of the first and divide the remainders by the value of the ratio $y / z$. Table 1 , beginning with column 6 , illustrates the procedure. It is not possible to start the transformations from column 4 , since these are the values for instantaneous duration and the ratio $y / z$ would be infinity. Negligible error will result, however, in computing the hydrograph for a finite time of very short duration, such as $\Delta t=0.02 T$, merely by averaging the successive ordinates, at intervals of $\Delta t$, of the instantaneous hydrograph. This has been done to obtain column 5 , the $0.02 T$ hydrograph. Column 6 is the summation cf column 5 . Column 7 is identical to column 6 , but has been lagged by an interval 
of $0.1 T$. Column 8 is the differences between columns 6 and 7 divided by $y / z=0.10 / 0.02=5$ and is the hydrograph for a duration $D / T=0.10$, or the $0.10 T$ hydrograph. The remaining columns in table 1 illustrate the computation of other hydrographs, all with $k / T$ values of 0.20 but with succeedingly longer values for $D / T$.

The question may be raised as to whether the transformations for $D / T$ should be made before, rather than after, the instantaneous translation hydrograph is routed through storage. The answer is that for linear models the choice is only a matter of convenience, for the final results will be the same regardless of the sequence of the computations. Routing computations are more laborious than $D / T$ transformatioms, and fewer routings are required if they are made before, rather than after, the transformations for $D / T$.

\section{NONLINEAR MODELS}

In nonlinear models, an instantaneous translation graph routed through nonlinear storage and then transformed to a particular $D / T$ unfortunately results in a graph different from that obtained from the same instantaneous translation graph transformed to the particular $D / T$ and then routed through the same nonlinear storage. In the sequence of their natural effects, duration is prior to storage; thus it is necessary in nonlinear models to make $D / T$ transformations to the translation graph before routing.

Transformations for the translation hydrograph may be made in a manner identical to the transformation described for linear models. Computations for the transformations to $D / T$ values of $0.1,0.2,0.5$, $0.7,1.0,1.5$, and 2.0 are given in table 2 . In this table the summation curve is given but once; the reader may obtain the appropriate value of the lagged summation curve by deducting $D / T$ from $H / T$, and th by reading the value from the indicated line of column 4 .

Translation hydrographs for these finite values of $D / T$ are plotted in figure 2. As $D / T$ approaches unity the shape of the graph approacl es that of the normal probability curve, and for values greater than unity, the graph approaches a trapezoid. The base of the graph, in all cases, is $(D / T)+1$, and the upper base of the trapezoid is $(D / T)-1$. The maximum ordinate is closely approximated by a simple relationship that changes at the point $D / T=1$. For smaller values of $D / T$ the relations is $Q_{m}=645.333(2.0-D / T)$; for higher values, $Q_{m}=645.333 /$ $(D / T)$.

For nonlinear storage developments, the appropriate finite trans ${ }^{1} a-$ tion hydrograph should be used, rather than the instantanec'is translation hydrograph. 
।

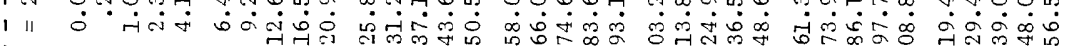

$1 \xi$ 1 a

Omom- m-4t

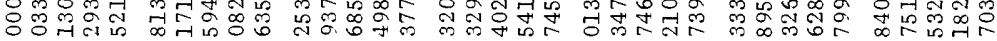
ثै

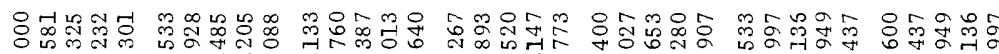

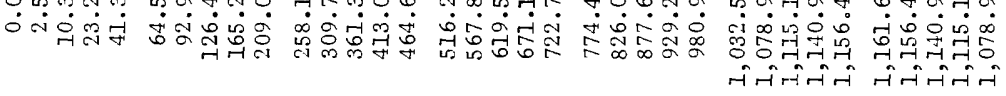

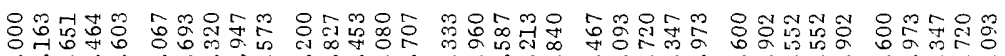

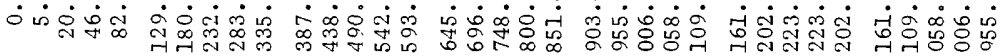

1 वे नी

向

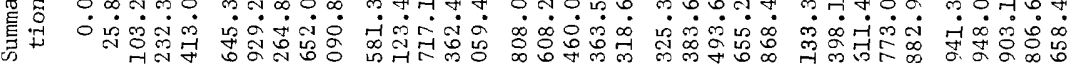

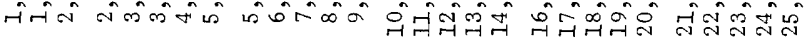

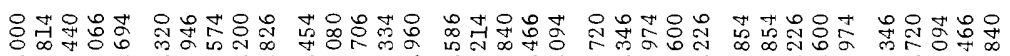

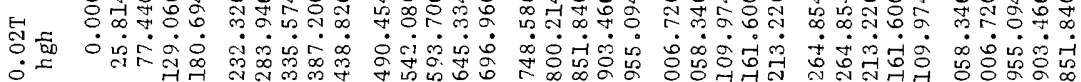

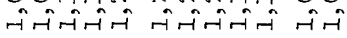

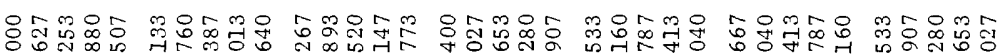

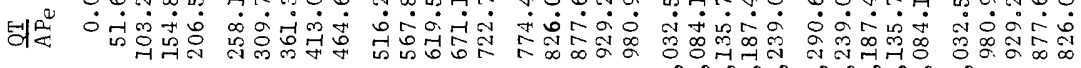

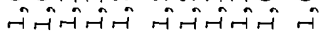

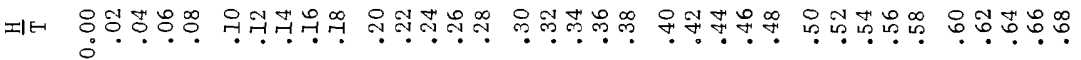


lᄋ

I I Jiণ

1

1

$1, \frac{1}{1}$

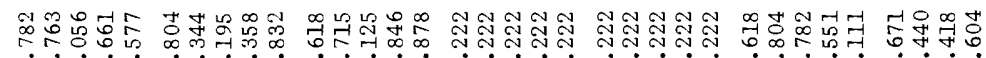

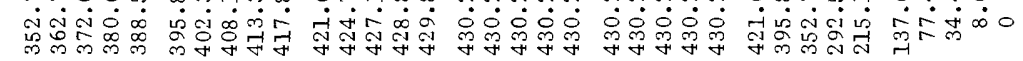
, 告

$1 \stackrel{1}{1}$

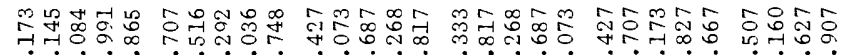

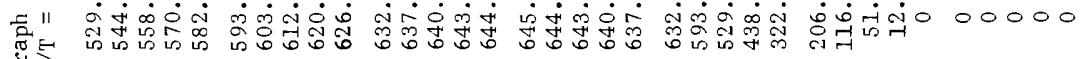

品吕

क्षे

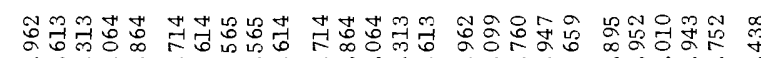

E

통

象

要

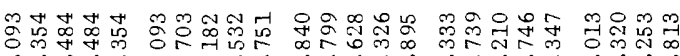
出 "I

1 은

$1: 0$

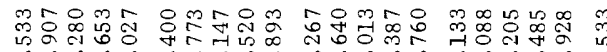

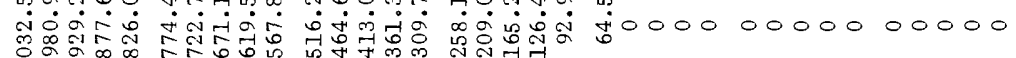
$-1$

1두

l II iิ

要离

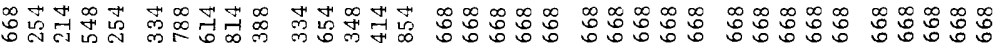

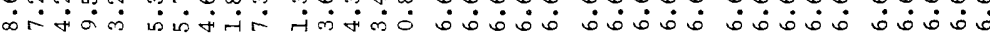

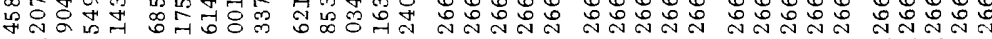

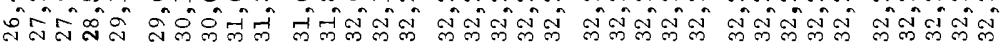

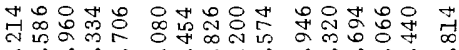

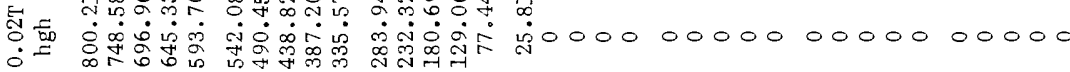

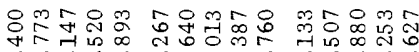

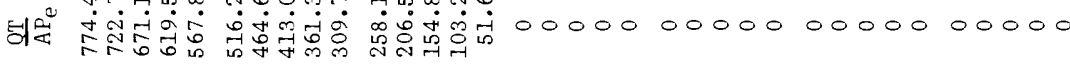

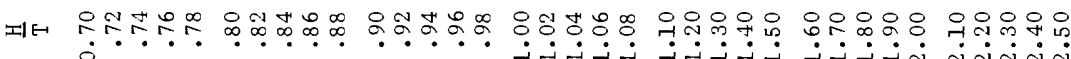




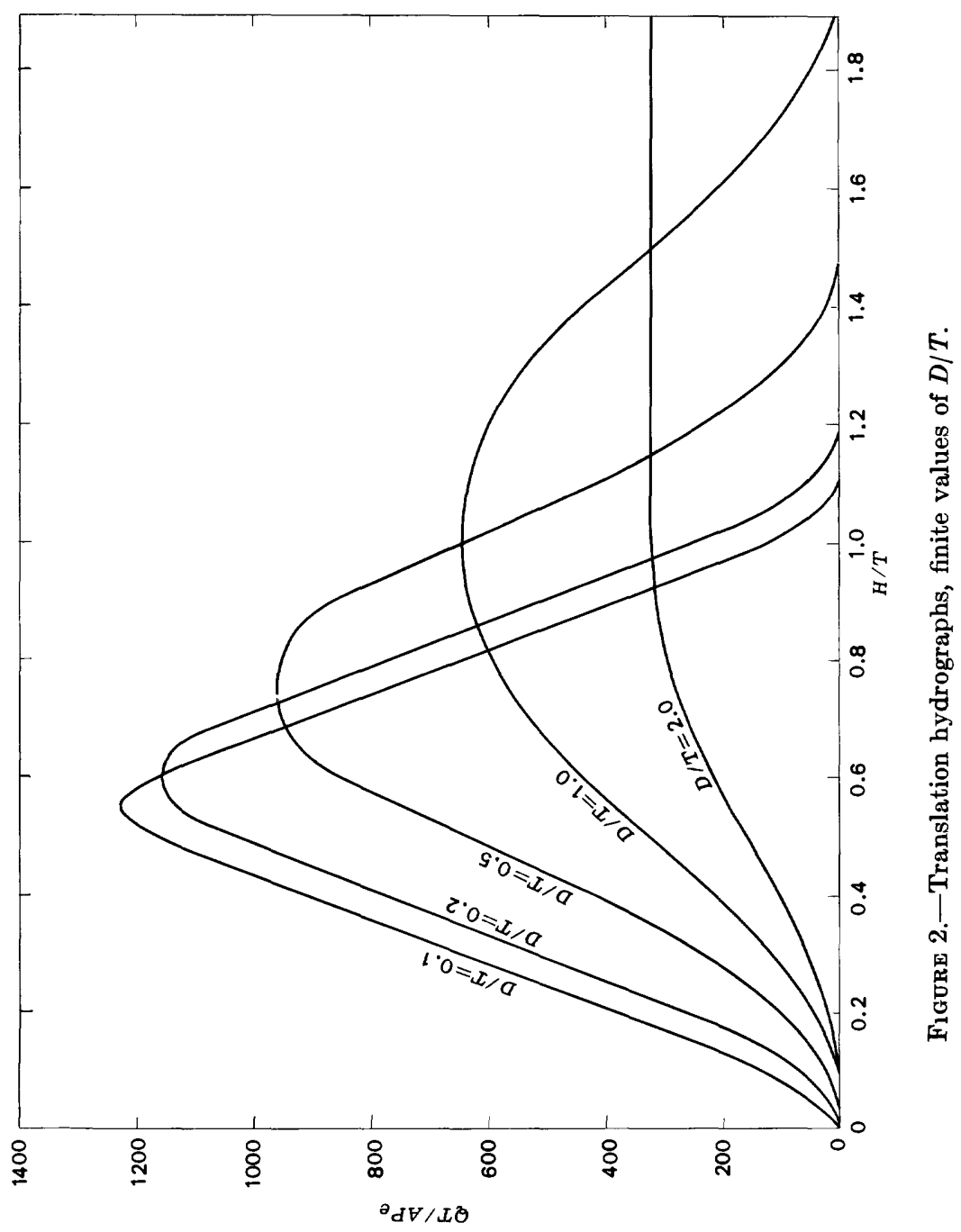




\section{MODEL HYDROGRAPHS}

The foregoing sections have presented the concept of the translation hydrograph, both instantaneous and finite, and have shown how these translation hydrographs may be modified by reservoir routing techniques to represent actual runoff hydrographs. It should again be pointed out, and emphasized, that by expressing these hydrographs in terms of dimensionless ratios, rather than absolute values, a comparatively few computations will provide a systematized set of tables; of modest extent, from which an almost limitless number of flood hydrographs readily may be computed. To compute a flood hydrograph, select the appropriate hydrograph from the tables and multiply all the values by appropriate simple constants which are functions of the time and areal characteristics of the specific drainage area. (This conversion process is described in detail in the section "Application to Specific Areas.") The systematized tables from which this final computation is made are presented in tables 3-36 and constitute the model hydrographs.

\section{LINEAR MODELS}

Tables 3-28 constitute the linear models. Each table is distinguished from all the others by its unique value of the ratio $k / T$, which ranges from 0.2 to 3.0. Values of this ratio have been chosen such that by using the table whose value is nearest to the actual $k / T$ ratio for a specific area, the tabular values will be within 5 percent of true values. Each table is similar to all the others in that it provides model hydrographs for various values of the ratio $D / T$ ranging from instantaneous to 2.0. To select the proper linear model, first compute $k / T$ to determine the appropriate table, then compute $D / T$ to determine the apr ${ }^{r}$ priate column within the table.

\section{NONLINEAR MODELS}

Tables 29-36 are by no means an exhaustive array of all possible nonlinear models, but they do appear to be a representative one. Ffor all these models, the storage equation for the reservoir routing is $S=k O^{1 / 2}$, which approximates the degree of nonlinearity most common in analyses of actual flood hydrographs. Further experience may demonstrate the need for a more extensive array of nonlinear models, but the present array is sufficient to illustrate the techniques for nonlinar computations and also sufficient to cover most of the problems so far encountered.

Each of the nonlinear models is distinguished from all the others by its unique value of the ratio $k / \sqrt{A P_{e} / T}$, which ranges from 2.0 to 12.0. Values of this ratio have been chosen such that by using the table whose value is nearest to the actual ratio for a specific area, the tabular 
values will be within 5 percent of the true values. Each table is similar to all the others in that it provides model hydrographs for various values of the ratio $D / T$, ranging from instantaneous to 2.0. To select the proper nonlinear model, first compute $k / \sqrt{A P_{e} / T}$, to determine the appropriate table; then compute $D / T$ to determine the appropriate column within the table.

\section{APPLICATION TO SPECIFIC AREAS}

All the model hydrographs, both linear and nonlinear, are expressed in terms of dimensionless ratios. To prepare a flood hydrc graph for a specific area, only the proper time and areal dimensions must be incorporated into the appropriate model.

For all models, the abscissa of the hydrograph is expressed in terms of $\mathrm{H} / T$, in which $H$ is hours from beginning of rainfall excess. To obtain the proper time expressions for any flood hydrograrh, multiply the values of $H / T$, as given in column 1 of all the tables, ky the value of $T$ for the specific area.

Likewise, for all models, the ordinates of the hydrograph are expressed in terms of $Q T / A P_{e}$. To obtain the proper discharge, in cubic feet per second, for any flood hydrograph, multiply the values of $Q T / A P_{e}$ by the value of $A P_{e} / T$ for the specific area.

The following examples, involving both linear and nonlirear models, will illustrate the procedures and indicate the accuracy of results.

\section{EXAMPLE 1}

A tributary to Second Creek, at Keptown (Effingham County), Ill., has a drainage area of $1.62 \mathrm{sq} \mathrm{mi}$. As determined by methods subsequently described, the storage is linear, the value of $k$ is $1.03 \mathrm{hr}$, and the value of $T$ is $1.52 \mathrm{hr}$. For the storm of July 15, 1؟57, rainfall excess of $0.87 \mathrm{in}$. occurred in $0.67 \mathrm{hr}$, beginning at $1045 \mathrm{hr}$. Compute the surface-runoff hydrograph from the appropriate model, and compare with the surface runoff from the observed hydrograph.

By using the values just given, $k / T=1.03 \mathrm{hr} / 1.52 \mathrm{hr}=0.68 ; D / T=$ $0.67 \mathrm{hr} / 1.52 \mathrm{hr}=0.44$. The appropriate model is taken from table 12 $(k / T=0.7)$, columns 1 and 6 . The value of $A P_{e} / T$ is $(1.62 \mathrm{sq} \mathrm{mi} \times 0.87$ in.) $/ 1.52 \mathrm{hr}=0.927 \mathrm{cfs}$. The model is converted by multiplying the values from column 1 by $1.52 \mathrm{hr}$ and the values from column 6 by $0.927 \mathrm{cfs}$.

Four graphs are shown in figure 3. The two light lines show the total observed runoff and the estimate for base flow. The dashed line is the difference between the first two graphs, or the actual surface runoff. This line should be compared with the heavy continuous line which is the converted model hydrograph. 


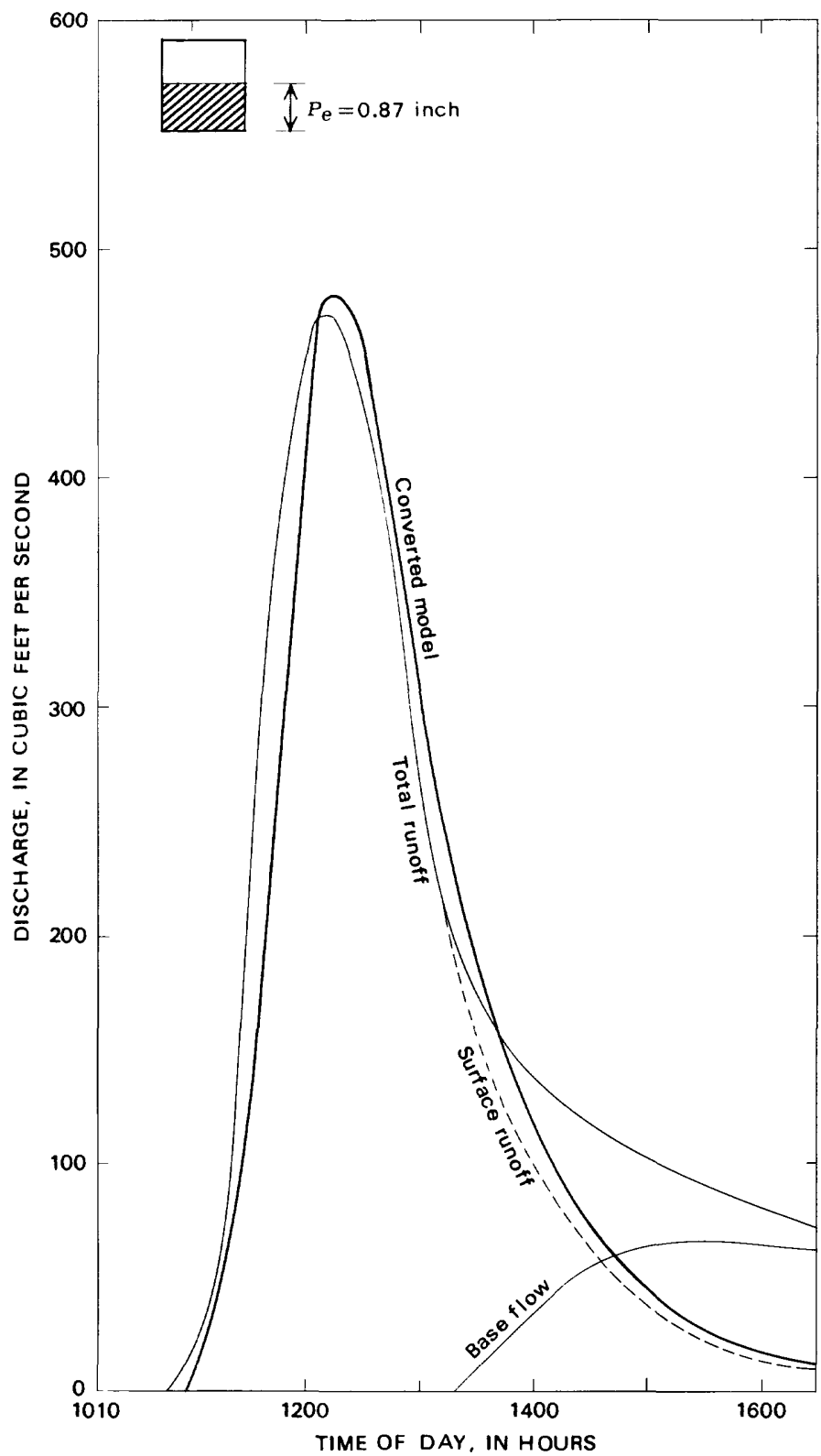

Figore 3.-Hydrographs for tributary to Second Creek at Keptown, Ill. 


\section{EXAMPLE 2}

A tributary to Kankakee River, near Bourbonnais (Kankakee County), Ill., has a drainage area of $0.192 \mathrm{sq}$ mi. As determined by methods subsequently described, the storage is linear, the value of $k$ is $0.405 \mathrm{hr}$, and the value of $T$ is $0.88 \mathrm{hr}$. For the storm of July 12, 1957, rainfall excess of 1.90 in. occurred in $0.75 \mathrm{hr}$, beginning at $1945 \mathrm{hr}$. Compute the surface-runoff hydrograph from the appropriate model, and compare with the surface runoff from the observed hydrograph.

$\mathrm{By}$, using the values just given, $k / T=0.405 \mathrm{hr} / 0.8 \varepsilon \mathrm{hr}=0.46$; $D / T=0.75 \mathrm{hr} / 0.88 \mathrm{hr}=0.85$. The appropriate model is taken from table $8(k / T=0.45)$, columns 1 and 10 . The value of $A P_{e} / T$ is 0.192 sq $\mathrm{mi} \times 1.90 \mathrm{in} . / 0.88 \mathrm{hr}=0.415 \mathrm{cfs}$. The model is converted by multiplying the values from column 1 by $0.88 \mathrm{hr}$ and the values from column 10 by $0.415 \mathrm{cfs}$.

Four graphs are shown in figure 4 . The two light lines show the total observed runoff and the estimate for base flow. The dashed line is the difference between the first two graphs, or the actual surface runoff. This line should be compared with the heavy continuous line, which is the converted model hydrograph.

\section{EXAMPLE 3}

Mazon River near Coal City (Grundy County), Ill., has a drainage area of $470 \mathrm{sq} \mathrm{mi}$. As determined by methods subsequently described, the storage is linear, the value of $k$ is $20 \mathrm{hr}$, and the value of $T$ is $30 \mathrm{hr}$. For the storm whose peak occurred on May 12, 1943, rainfall excess of $1.79 \mathrm{in}$. occurred in $20 \mathrm{hr}$, beginning at $1600 \mathrm{hr}$ on May 10. Compute the surface-runoff hydrograph from the appropriate model, and compare with the surface runoff from the observed hydrograph.

By using the values just given, $k / T=20 \mathrm{hr} / 30 \mathrm{hr}=0.67 ; D / T=20$ $\mathrm{hr} / 30 \mathrm{hr}=0.67$. The appropriate model is taken from table $12(k / T=$ 0.7 ), columns 1 and 9 . The value of $A P_{e} / T$ is $470 \mathrm{sq} \mathrm{mi} \times 1.79 \mathrm{in} . / 30$ $\mathrm{hr}=28.0 \mathrm{cfs}$.

Four graphs are shown in figure 5. The two light lines show the total observed runoff and the estimate for base flow. These two graphs are the same as those shown in the report by Mitchell (1948, p. 115). The dashed line is the difference between the first two graphs, or the actual surface runoff. This line should be compared with the heavy continuous line, which is the converted model hydrograph.

\section{EXAMPLE 4}

A tributary to Vermilion River, at Lowell (La Salle County), Ill. has a drainage area of $0.126 \mathrm{sq} \mathrm{mi}$. As determined by methods subsequently described, the storage is nonlinear $(x=0.5)$, the ralue of $k$ is 


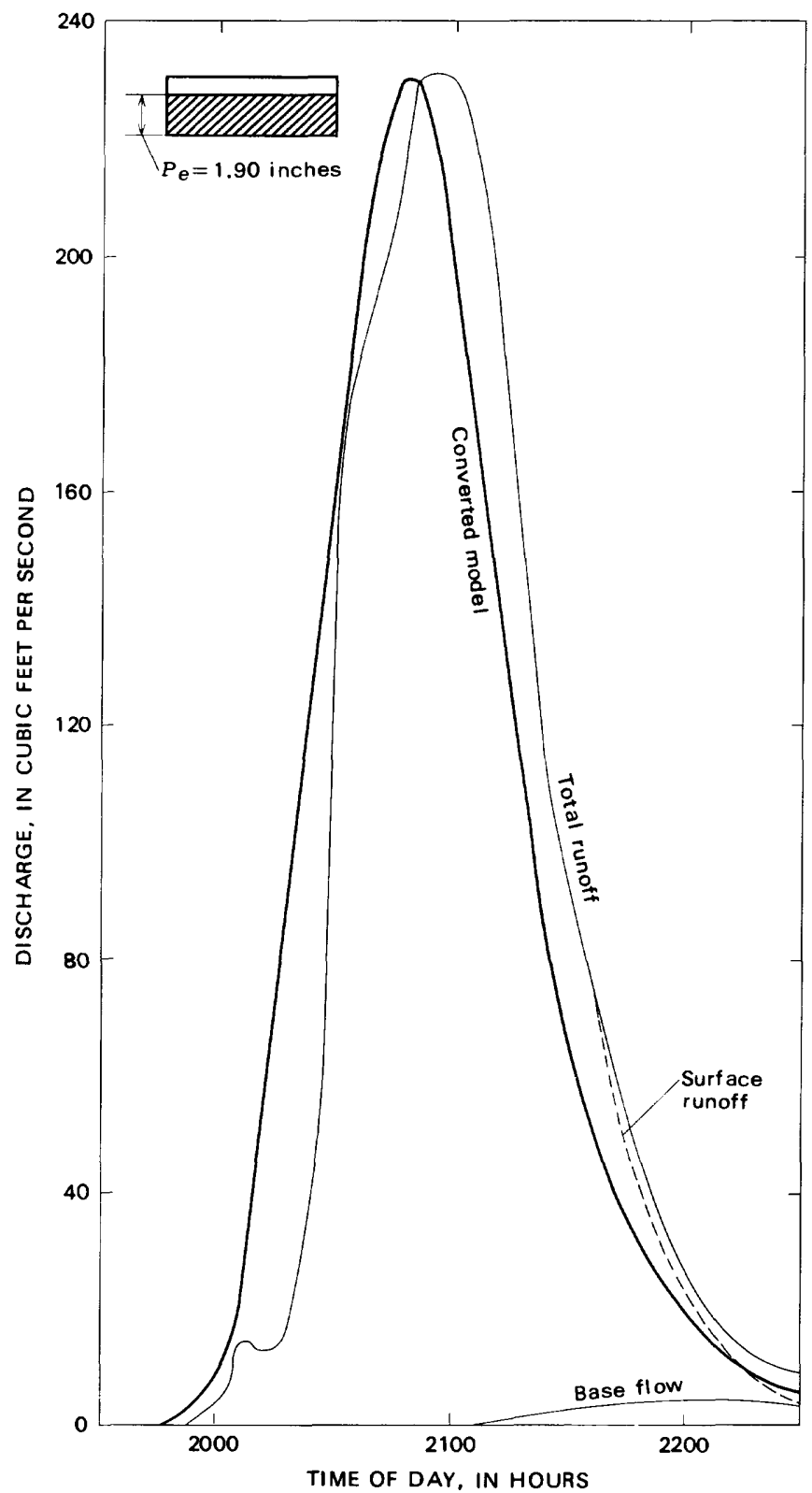

Figure 4.-Hydrographs for tributary to Kankakee River near Bourbonnais, Ill. 


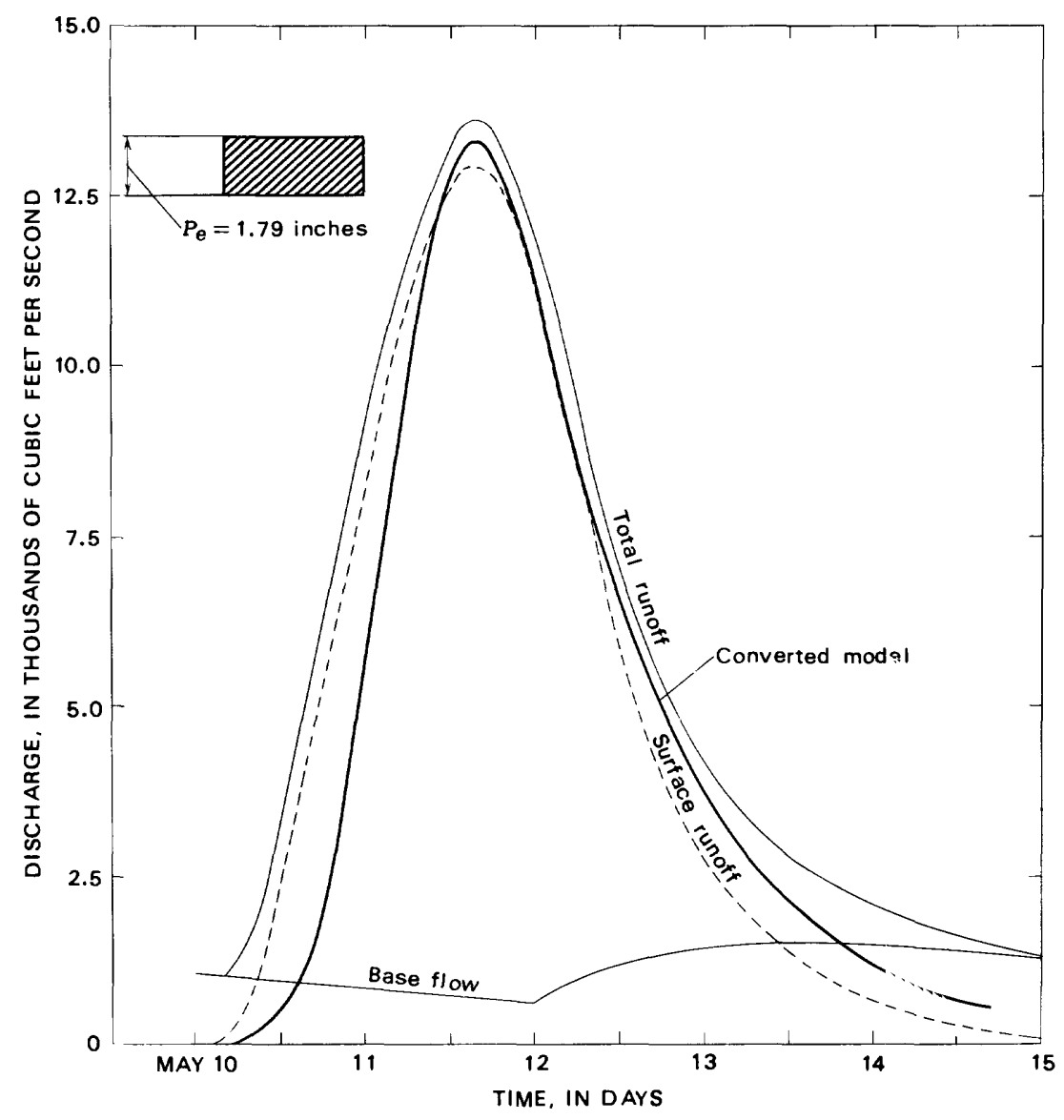

Figdre 5.-Hydrographs for Mazon River near Coal City, Ill.

$1.34 \mathrm{hr}$, and the value of $T$ is $0.32 \mathrm{hr}$. For the storm of June 12, 1957, rainfall excess of $0.045 \mathrm{in}$. occurred in $0.067 \mathrm{hr}$, beginning at $0625 \mathrm{hr}$. Compute the surface-runoff hydrograph from the appropriate model, and compare with the surface runoff from the observed hydrograph.

By using the values just given, $k / \sqrt{A P_{e} / T}=1.34 / \sqrt{(0.126 \times 0.045)} /$ $\overline{0.32}=10.1 ; D / T=0.067 \mathrm{hr} / 0.32 \mathrm{hr}=0.2$. The appropriate model is taken from table $34\left(k / \sqrt{A P_{e} / T}=10.0\right)$, columns 1 and 4 . The value of $A P_{e} / T$ is $0.126 \mathrm{sq} \mathrm{mi} \times 0.045 \mathrm{in} . / 0.32 \mathrm{hr}=0.018 \mathrm{cfs}$. The model is converted by multiplying the values from column 1 by $0.32 \mathrm{hr}$ and the values from column 4 by $0.018 \mathrm{cfs}$.

Four graphs are shown in figure 6 . The two light lines show the total observed runoff and the estimate for base flow. The dashed line is the difference between the first two graphs, or the actual surface runoff. 

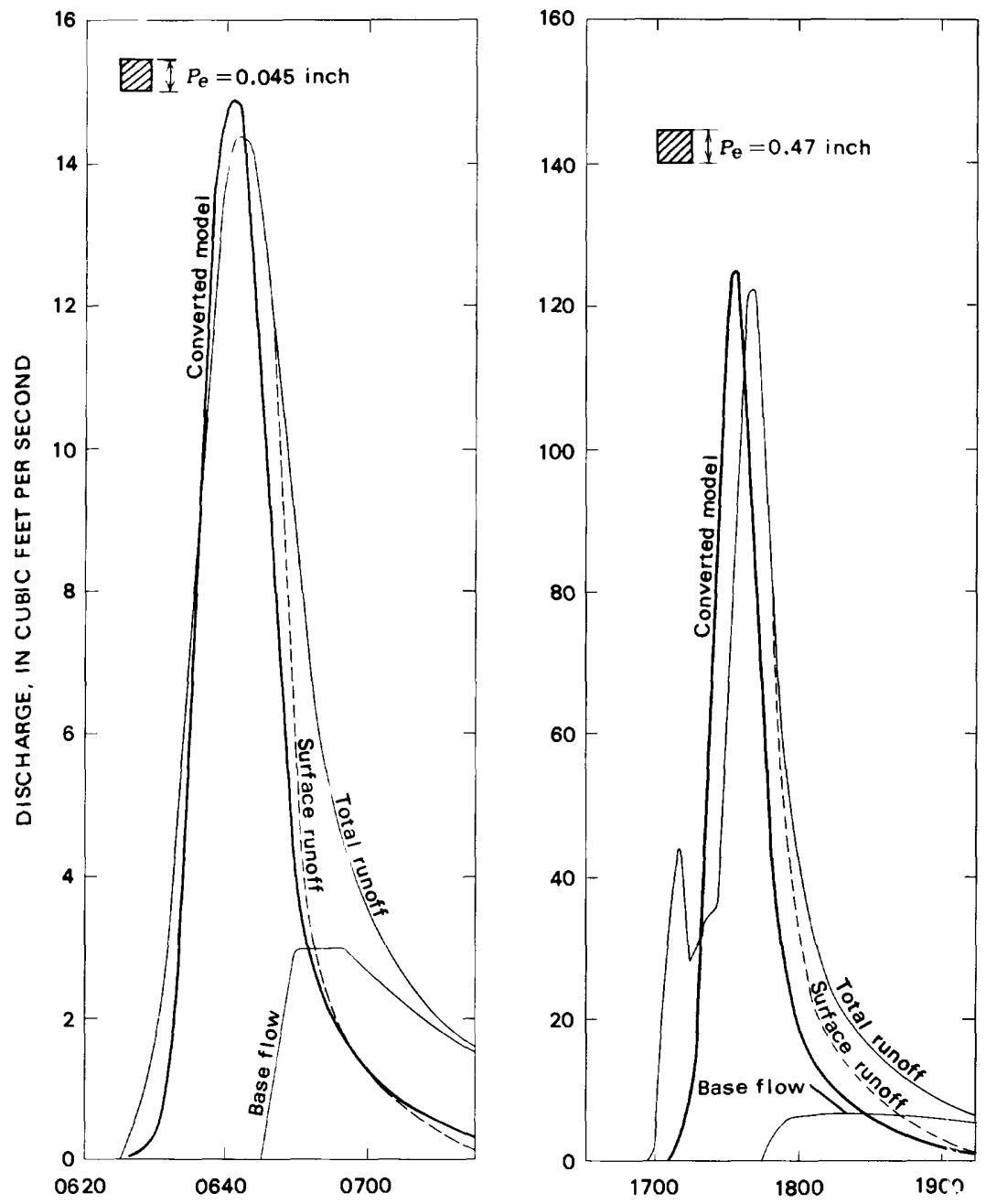

TIME OF DAY, IN HOURS

Figure 6.-Hydrographs for tributary to Vermilion River at Lowell, Ill. (left) and for tributary to Mud Creek near Tower Hill, Ill., (right).

This line should be compared with the heavy continuous line, which is the converted model hydrograph.

\section{EXAMPLE 5}

A tributary to Mud Creek, near Tower Hill (Shelby County), Ill., has a drainage area of $0.204 \mathrm{sq}$ mi. As determined by methods subsequent'y described, the storage is nonlinear $(x=0.5)$, the value of $k$ is $4.40 \mathrm{hr}$, and the value of $T$ is $0.58 \mathrm{hr}$. For the storm of June 19, 1c.56, 
rainfall excess of $0.47 \mathrm{in}$. occurred in $0.25 \mathrm{hr}$, beginning at $1700 \mathrm{hr}$. Compute the surface-runoff hydrograph from the appropr:ate model, and compare with the surface runoff from the observed hydrograph.

By using the values just given, $k / \sqrt{A P_{e} / T}=4.40 / \sqrt{(0.204 \times 0.47) /}$ $\overline{0.58}=10.8 ; D / T=0.25 \mathrm{hr} / 0.58 \mathrm{hr}=0.43$. The appropriate model is taken from table $35\left(k / \sqrt{A P_{e} / T}=11.0\right)$, columns 1 and 6 . The value of $A P_{e} / T$ is $0.204 \mathrm{sq} \mathrm{mi} \times 0.47 \mathrm{in} . / 0.58 \mathrm{hr}=0.165 \mathrm{cfs}$. The model is converted by multiplying the values from column 1 by $0.58 \mathrm{hr}$ and the values from column 6 by $0.165 \mathrm{cfs}$.

Four graphs are shown in figure 6. The two light lines show the total observed runoff and the estimate for base flow. The dashed line is the difference between the first two graphs, or the actual surface runoff. This line should be compared with the heavy continous line, which is the converted model hydrograph.

\section{EXAMPLE 6}

Assume that for example 5 the rainfall excess had besn 1.00 in. and that all other factors were as previously given. After selecting the appropriate model, compute the peak discharge of surface runoff.

By using the values given, $k / \sqrt{A P_{e} / T}=4.40 / \sqrt{(0.204 \times 1.00) / 0.58}=$ 7.4; $D / T=0.43$, as before. The appropriate model is taken from table $31\left(k / \sqrt{A P_{e} / T}=7.0\right)$, columns 1 and 6 . The value of $A P_{e} / T$ is 0.204 sq $\mathrm{mi} \times 1.00 \mathrm{in} . / 0.58 \mathrm{hr}=0.352 \mathrm{cfs}$. The model indicates the.t the peak discharge will occur at $0.8 \times 0.58 \mathrm{hr}=0.46 \mathrm{hr}$ after beginning of rainfall excess and will have a magnitude of $914.0 \times 0.352 \mathrm{cfs}=322 \mathrm{cfs}$.

This last example has been included to illustrate the fect that for nonlinear storage peak discharges are not proportional to amount of rainfall excess. A comparison of examples 5 and 6 shows that an increase in rainfall excess of 113 percent (from 0.47 in. to $1.00 \mathrm{in}$.) produced an increase in peak discharge of 158 percent (from 125 cfs to $322 \mathrm{cfs}$.)

\section{DETERMINATION OF HYDROGRAPH PARAMETERS}

Two types of phenomena combine to produce the flood hydrograph. One of these is the meteorological event-the amount, duration, and distribution of rainfall. The other is the physiographic character of the drainage basin. For the most part, past meteorological events are only a rough and uncertain guide to future intensities and durations. Also, barring the advent of bulldozers (and neglecting the ephemeral effects of temperature and antecedent precipitation), the physiographic character of a drainage basin changes only imperceptibly, from day to day, and from decade to decade. ("Eternal as the hills" is a realistic expression.) Thus, although successive flood hydrographs for a given basin may vary greatly because of the variations in the meteorological 
events, they will nevertheless have certain invariable characteristics, reflecting the relatively constant, unchanging physiography of the basin. These characteristics, once accurately determined for a basin, may be applied with confidence to a synthesis of other hydrographs for that basin.

The hydrograph does not, of course, provide direct information on discrete elements of the physiography, such as area, slope, or stream density. In fact, for area, the hydrograph provides no information whatsoever; this must be obtained from other sources, such as topographic maps. However, information for the other physiographic factors which influence the hydrograph is available in a total, integrated form as certain constant (or at least nearly constant) hydrograph parameters. These parameters are the characteristic time, $T$ and the storage constants, $k$ and $x$.

\section{DETERMINATION FROM OBSERVED HYDROGRAPHS}

The most direct, and most satisfactory, method of determination of hydrograph parameters is appropriate analysis of observed hydrographs. All three of the significant parameters, $T, k$, and $x$, may be determined by such analysis. The principal shortcoming: of this method is the lack of observed hydrographs for ungaged areas.

\section{GHARACTERISTIG TIME, $T$}

In the discussion of translation flow (section "Translation Hydrograph"), $T$ was considered to be the time required for flow to arrive at the outlet from the most distant point of the drainage basin. Many hydrologists have assumed, although none has rigorously proved, that this is the time from ending of rainfall excess to the point of inflection on the recession side of the hydrograph. Even in the absence of rigor ous proof, experience has demonstrated repeatedly that this lapse of time is a constant for a given basin and adequately serves the purposes for which the concept of $T$ was intended.

The characteristic time, $T$, for any given basin is determined by subtracting the time of ending of rainfall excess from the time of occurence of the inflection (change of curvature from concave downward to concave upward) on the recession limb of the hydrograpl.

\section{STORAGE GONSTANTS, $k$ AND $x$}

In view of the assumption upon which the model hydrographs are developed - that the hydrograph of surface runoff is a translation hydrograph modified by reservoir storage, $S=k O^{x}$-appropriate analysis of the hydrograph should disclose the values of $k$ and $x$. For linoar storage, the value of $x$ is, of course, unity, and a procedure for deter- 
mining $k$ already has been published (Mitchell, 1962, p. 23). Briefly, this procedure involves computation of a recession coefficient, $r$, for any convenient time, $\Delta t$, and substitution in the formula

$$
k=\Delta t(1+r) / 2(1-r) .
$$

When storage is linear, $r$ has the same value for all points equally spaced in time on a recession curve, but when storage is nonlinear, the value of $r$ will vary with the discharge. Hence, to determine the value of $k$, as well as $x$, for nonlinear storage a new procedure is required.

The formula for $k$, just described, was developed from the floodrouting equation

$$
O_{2}=\left(2 S_{1} / \Delta t\right)-O_{1}-\left(2 S_{2} / \Delta t\right)+\left(I_{1}+I_{2}\right)
$$

(see section "Routing Considerations") and the assumption that if beyond the point of inflection on the recession limb of the lydrograph, inflow has been completed, both $I_{1}$ and $I_{2}$ are zero. Starting with this same assumption, Shen (1962) found that both $k$ and $x$ could be evaluated by the following procedure: On logarithmic coordinates, plot $\Delta Q / \Delta t$ as ordinate and average $Q$ as abscissa, fit a straight line to these points, and determine the slope and the intercept (at $Q=1$ ); to obtain the value of $x$, subtract the slope of the line from 2.0 , and to obtain $k$, multiply the intercept by $x$ and take the reciprocal of this product.

Figure 7 shows examples of Shen's procedure. The points plotted there are derived from routed graphs which appear later as figures 11, 12 , and 13. Some of the points are from the curves for 1 inch of runoff, others from the curves for 2 inches of runoff; alinenent of the points is independent of this variation. One curve is horizontal (zero slope), with an intercept of 50 . Hence, the value of $x$ is $2-O=2$, the value of $k$ is $1 /(2 \times 50)=0.01$, and the storage equation is $S=0.01 O^{2}$. For the second line, the slope is 1.0 and the intercept is 5.0 ; so $x=2-1$ $=1, k=1 /(1 \times 5)=0.20$, and the storage equation is $S=0.20$. For the steepest line, the slope is 1.5 and the intercept is 0.2 ; so $x=2.0$ $-1.5=0.5, k=1 /(0.5 \times 0.2)=10$, and the storage equatior is $S=10.0$ $0^{0.5}$

Shen's method is further illustrated by figure 8 . The five sets of data plotted here are for the same five stations that were used to describe the application of model hydrographs to specific areas.

The open circles are for the tributary to Second Creek at Keptown, Ill., and for the storm of July 15, 1957. The values of actual surface runoff (the dashed line of fig. 3) were used to compute tl ese points. 


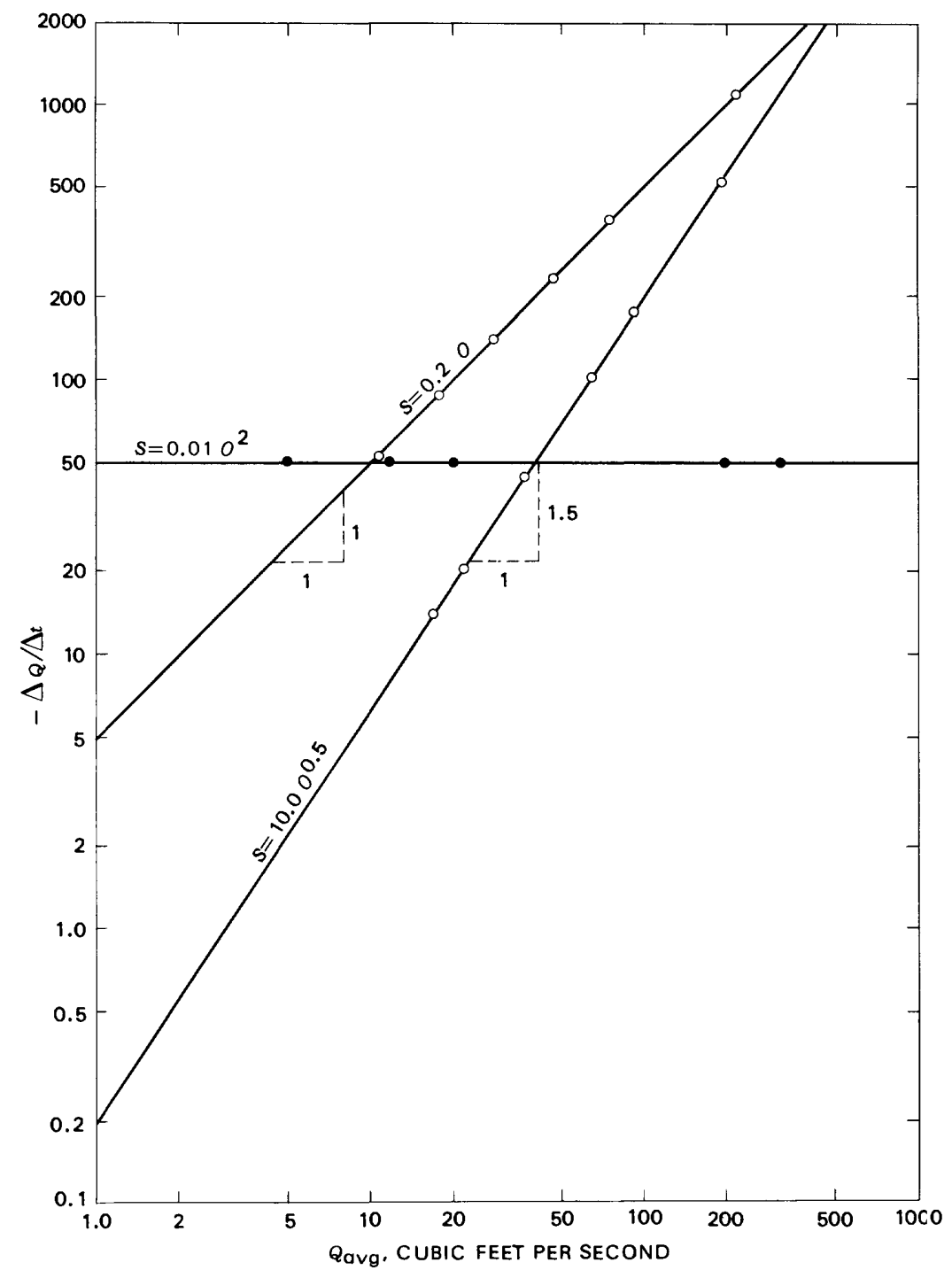

Figure 7.-Analyses for storage constants of model hydrographs.

Slope of the line is 1.0 , and the intercept is 0.94 ; thus the constants are $x=1.0$, and $k=1.06$. (Determinations for other storms, not shown here, give an average value for $k$ of 1.03, as used in "Example 1.")

The solid circles are for the tributary to Kankakee River near Bourbonnais, Ill., and for the storm of July 12, 1957. The values of actual surface runoff (the dashed line of fig. 4) were used to compite these points. Slope of the line is 1.0 , and the intercept is 2.8 ; th us the constants are $x=1.0$ and $k=0.357$. (Determinations for other 


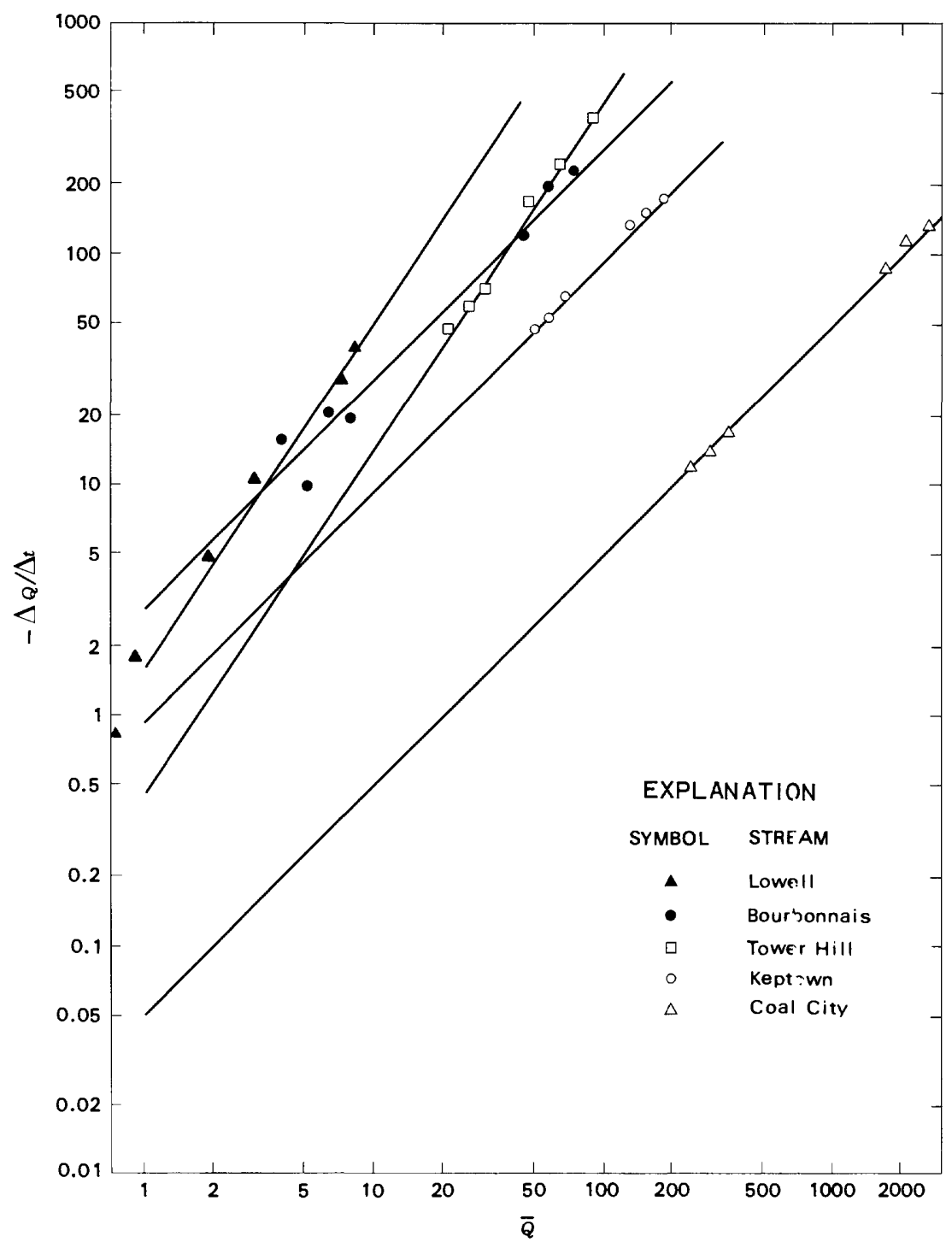

FIGURE 8.-Analyses for storage constants of observed hydrc?raphs.

storms, not shown here, give an average value for $k$ of 0.405 , as used in "Example 2.")

The open triangles are for Mazon River near Coal City, Ill. The published unit hydrograph (Mitchell, 1948) was used to compute these points. Slope of the line is 1.0 , and the intercept is 0.05 ; thus the constants are $x=1.0$ and $k=20$, as used in "Examplo. 3." 
The solid triangles are for the tributary to Vermilion River at Lowell, 1ll., and for the storm of June 12, 1957. The values of actual surface runoff (the dashed line of fig. 6) were used to compute these points. Slope of the line is 1.5 , and the intercept is 1.5 ; thus the constants are $x=0.5$ and $k=1.34$, as used in "Example 4."

The open squares are for the tributary to Mud Creek near Tower Hill, Ill., and for the storm of June 19, 1956. The values of actual surface runoff (the dashed line of fig. 6) were used to compute these points. Slope of the line is 1.5 , and the intercept is 0.45 ; thus the constants are $x=0.5$, and $k=4.40$, as used in "Example 5."

From these examples, which initially were chosen with other objectives in view, it should not be assumed that all observed hydrographs will result in plots for which slope of the lines will be precisely 1.0 or 1.5. Present knowledge concerning nonlinear hydrographs is so meager that slopes cannot be predicted. Our present experience indicates that for most of these plots the slopes will be near to 1.0 and that for the few others the slopes will tend to cluster about 1.5; but any slope appears to be possible. Thus, because a better understanding of nonlinearity is needed and models presently are available only for the two cases cited, the lines for all analyses should be drawn with slopes of either 1.0 (when possible) or 1.5 (when necessary).

\section{DETERMINATION FROM BASIN CHARACTERISTICS}

The storage effect results from physiographic influences. Therefore, the expression for a hydrograph may be correlated with the observable physical characteristics of its drainage basin.

Efforts have been made by means of multiple regression analyses to relate $T$ and $k$ for basins having linear storage characteristics to observed values of area $(A)$, length $(L)$, slope between point: 10 percent and 85 percent of the distance along the channel from the site to the divide $\left(S_{10 / 85}\right)$, soils infiltration index $\left(S_{i}\right)$, a surface-storage index giving percentage of the basin in lakes, ponds, and swamps $\left(S_{t}\right)$, percentage of basin covered by forest $\left(F^{\prime}\right)$, rainfall intensity $\left(I_{24,2}\right)$, and drainage density $\left(D_{d}\right)$. Many of the drainage basins lave no forest cover or surface storage; so a constant of 0.5 was added to these two basin characteristics to prevent the computer from stopping when attempting to take logarithms of zero values. Data for 48 stations were used in the regressions for $T$, and data for 43 stations were used in the regressions for $k$. The initial array of data follcwed the general arrangement described by

and

$$
T=f\left(A, L, S_{10 / 85}, S_{i}, S_{t}, F, I_{24,2}, D_{d}\right)
$$

$$
\underset{446-3 \pi 50-72-5}{k} f\left(A, L, S_{10 / 85}, S_{i}, S_{t}, F, I_{24,2}, D_{d}\right) .
$$


Stepwise multiple regression performed on these data indicated that only three independent variables were significant at either the 1-percent or 5-percent level. The general equations are

and

$$
T_{c}=C_{t} L^{a} S_{10 / 85}^{b} S_{t}^{c}
$$

where

$$
k_{c}=C_{k} L^{d} S_{10 / 85}^{e} S_{t}^{f}
$$

$T_{c}=$ computed lag time,

$k_{c}=$ computed storage time,

$C_{t}, C_{k}=$ regression constants,

$L=$ length of stream, in miles,

$S_{10 / 85}=$ slope, in feet per mile,

$S_{t}=$ surface-storage index +0.5 , in percent,

$a, b, c, d, e, f=$ regression coefficients.

The following table gives the regression coefficients, significant at the 1-percent level, to be used in the above equations and also indicates the coefficient of correlation and standard errcr of each equation:
0.837
0.837
5. 02
5. 33
0. 898
.650
.602

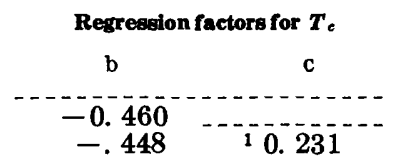
Coefficient of Standard error
correlation (percent)
$0.885 \quad 56.2$
$.929 \quad 44.4$

\begin{tabular}{|c|c|c|c|c|c|}
\hline \multirow{3}{*}{$\begin{array}{l}\text { }{ }^{\mathrm{C}_{\mathrm{k}}} \\
\text { 67. } 2 \\
\text { 16. } 5 \\
\text { 17. } 6\end{array}$} & & \multicolumn{2}{|c|}{ Regression factors for $k_{c}$} & \multirow{3}{*}{$\begin{array}{c}\text { Coefficient of } \\
\text { correlation } \\
0.921 \\
.959 \\
.970\end{array}$} & \multirow{3}{*}{$\begin{array}{c}\text { Standard error } \\
(\text { percent }) \\
48.9 \\
35.4 \\
30.6\end{array}$} \\
\hline & & -1.20 & $t$ & & \\
\hline & $\begin{array}{r}0.392 \\
.339\end{array}$ & $\begin{array}{l}1.80 \\
-.873 \\
-.860\end{array}$ & 0.258 & & \\
\hline
\end{tabular}
$.939 \quad 41.9$

The equations giving the best estimates of $T$ and $k$ were used to compute $T_{c}$ and $k_{c}$ for those stations used in the regression analyses. Figures 9 and 10 show the extent of agreement between the computed and actual values.

Data for more stations are being continually added to those data for stations described in the previous section. It is hoped that continued study based on these additional data and minor refinements to those existing will eventually lead to more accurate correlations.

For nonlinear storage effects, the data are, as yet, too few to warrant any attempt at correlations.

\section{UNIT HYDROGRAPH}

Many hydrologists now regard the unit hydrograph as a translation hydrograph modified by some element of reservoir-type storage. Only 


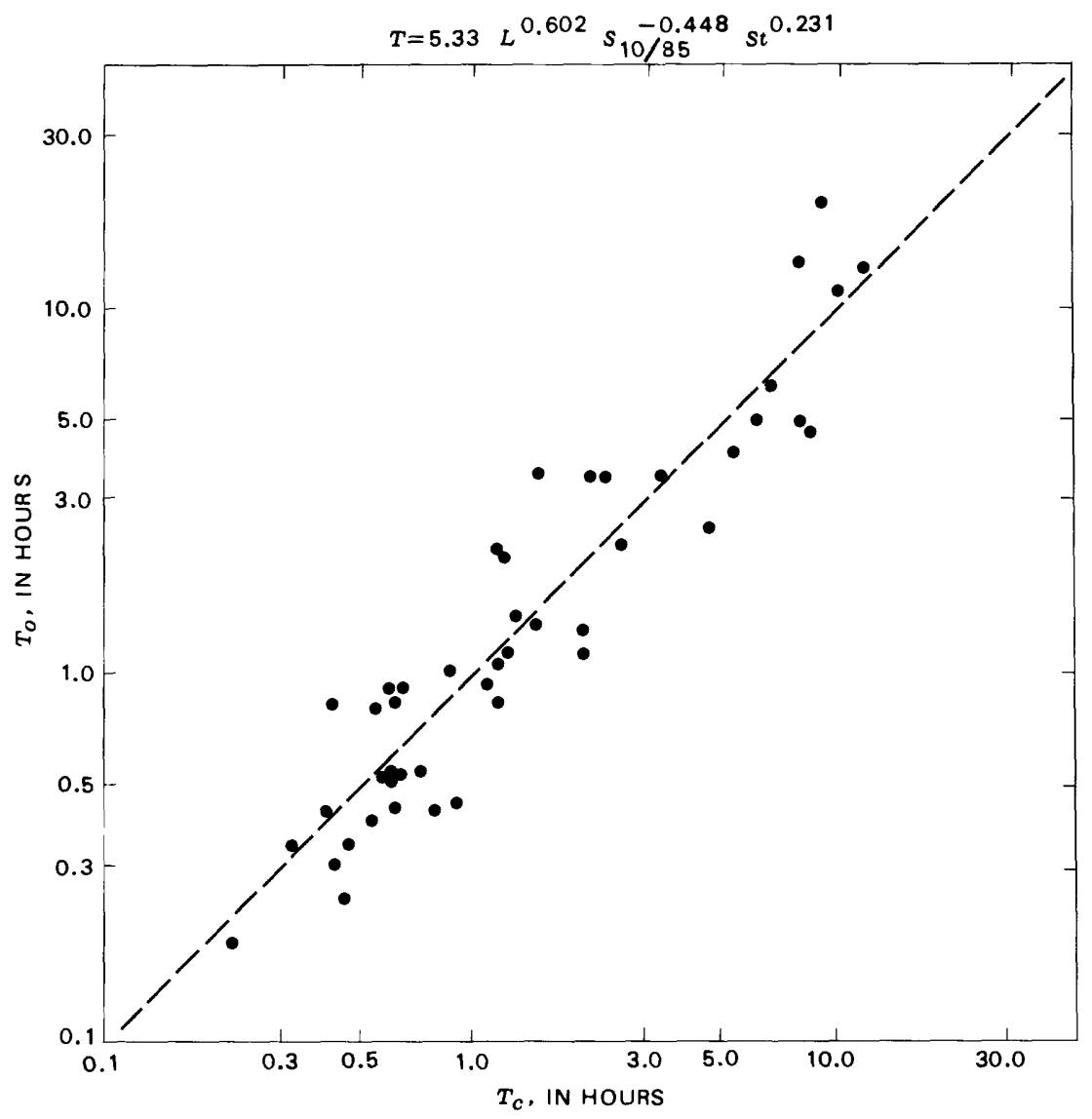

Frgure 9.-Observed, $T_{o}$, versus computed, $T_{c}$, values of $T$.

recently, however, has there been a realization that to define a true unit hydrograph, the reservoir storage must be a particular type, namely linear storage.

A principle of the unit-hydrograph technique is that for rainfall excesses uniformly distributed over a given area and within a given time, the ordinates of the graph of surface runoff are in linear prcnortion to the amounts of the rainfall excesses. This principle requires that the storage effect be a linear one. A rigorous mathematical development of this statement is not presently available, but a demonstration is quite simple.

In figure 11, the lower curve results from routing the translation hydrograph of figure 1 through linear storage, $S=0.200$. (See table 3 , cols. 1, 2.) Suppose now that for figure $1 P_{e}$ had been in units of "double inches," of sixths of a foot, rather than twelf ths. In that case, the ordinates of figure 1 would all have been doubled. The higher 


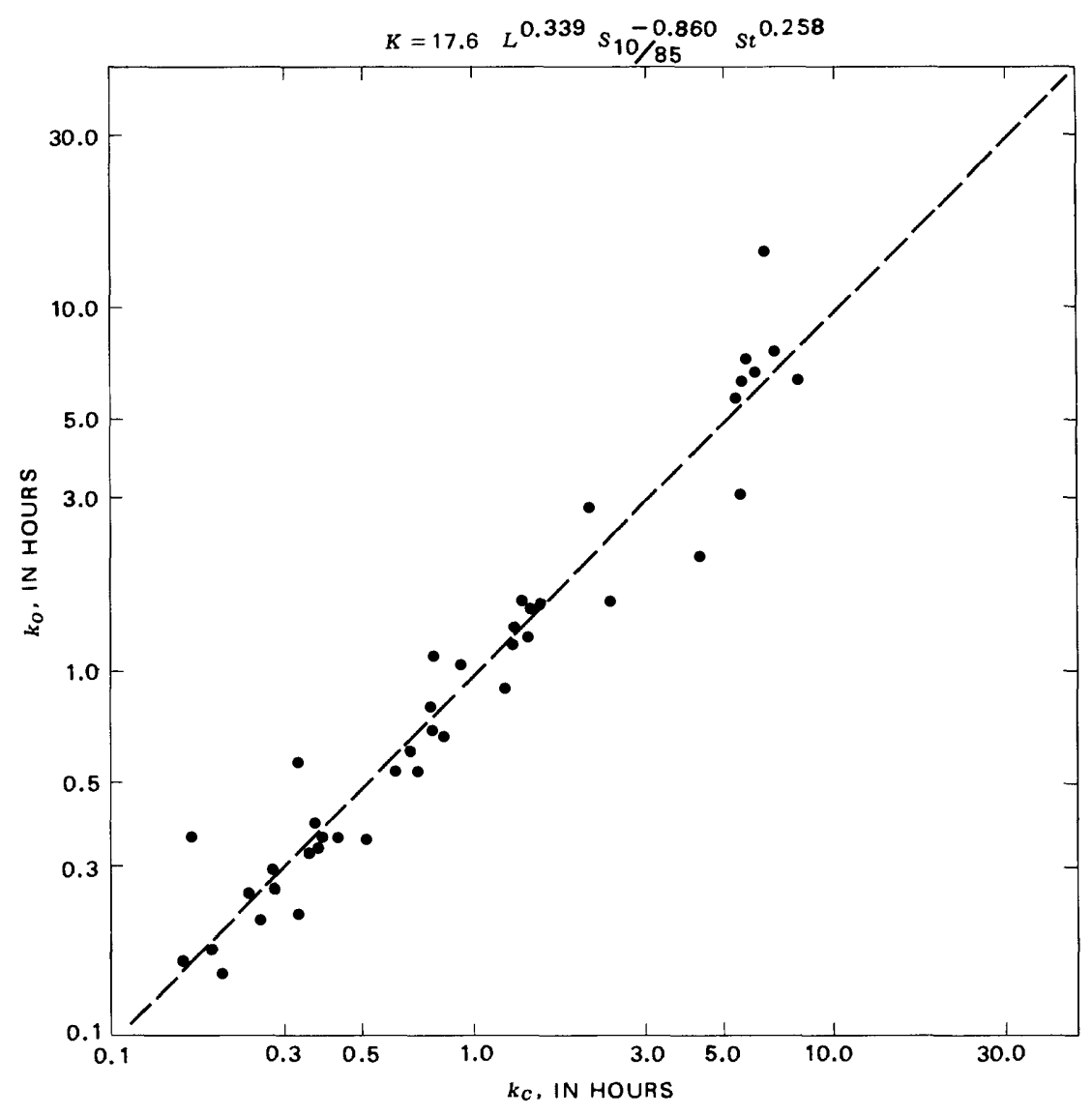

Figure 10.--Observed, $k_{o}$, versus computed, $k_{c}$, values of $k$.

curve in figure 11 results from routing these doubled ordinates through the same linear storage, $S=0.200$. Comparing the curves cf figure 11 shows that all ordinates of the second curve are twice those of the first. This relation meets the requirement of the unit-hydrograph principle that ordinates of the unit hydrograph be in linear proportion to rainfall excess.

In figure 12 , the same two translation hydrographs have been routed through nonlinear storage, $S=10 O^{0.5}$, to obtair the two graphs. Obviously there is no constant of proportionality between the ordinates of these graphs; hence they cannot be true unit hydrographs.

In figure 13, the same translation hydrographs have been routed through nonlinear storage, $S=0.01 O^{2}$. Again, the resulting graphs have no constant of proportionality, and thus fail to meet the specifications for true unit hydrographs. 


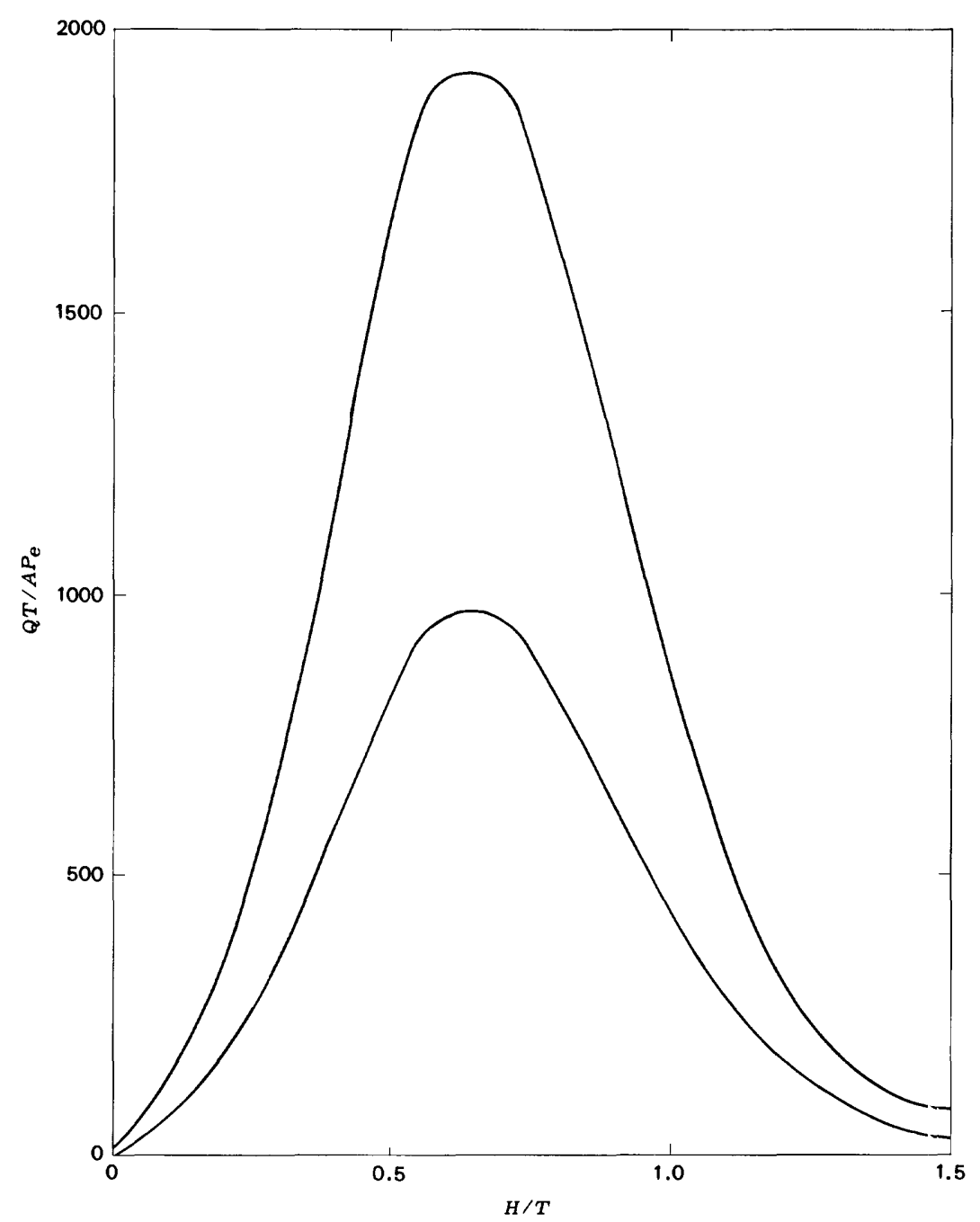

FIGURE 11.-Proportional graphs, resulting from routing through linear stor?ge, $S=0.20 O$.

A quick method for testing linearity of storage effect in any hydrograph is to plot it semilogarithmically and note the shape of the recession limb. If the value of $x$ is greater than unity, the recession will be concave downward; if less than unity, concave upward. Only when this recession is a straight line is the storage effect linear, and only then is the graph a true unit hydrograph. These statements are illustrated in figure 14, which shows the semilogarithmic plot for the recession limbs of the curves of figures 11,12 , and 13 . 


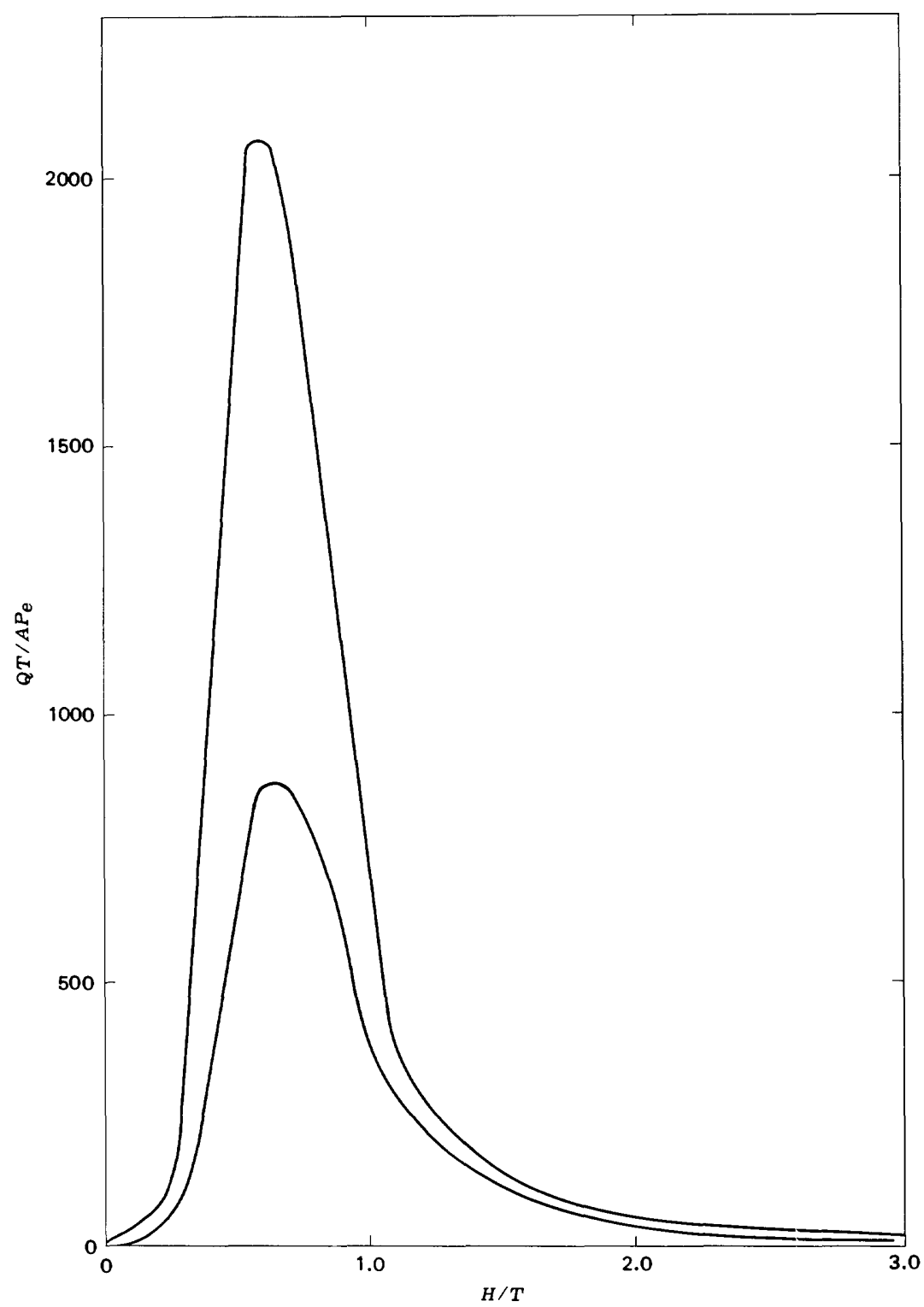

Figure 12.-Nonproportional graphs, resulting from routing through nonlinear storage $S=10 O^{0.5}$.

It is interesting to note the extent to which some of the so-called unit hydrographs in the published literature conform to the criterion of straight-line recession. Figure 15 shows some typical examples (from Mitchell, 1948). Figure 16 shows the hydrograph derived from observed data for Money Creek above Lake Bloomington, Ill., 


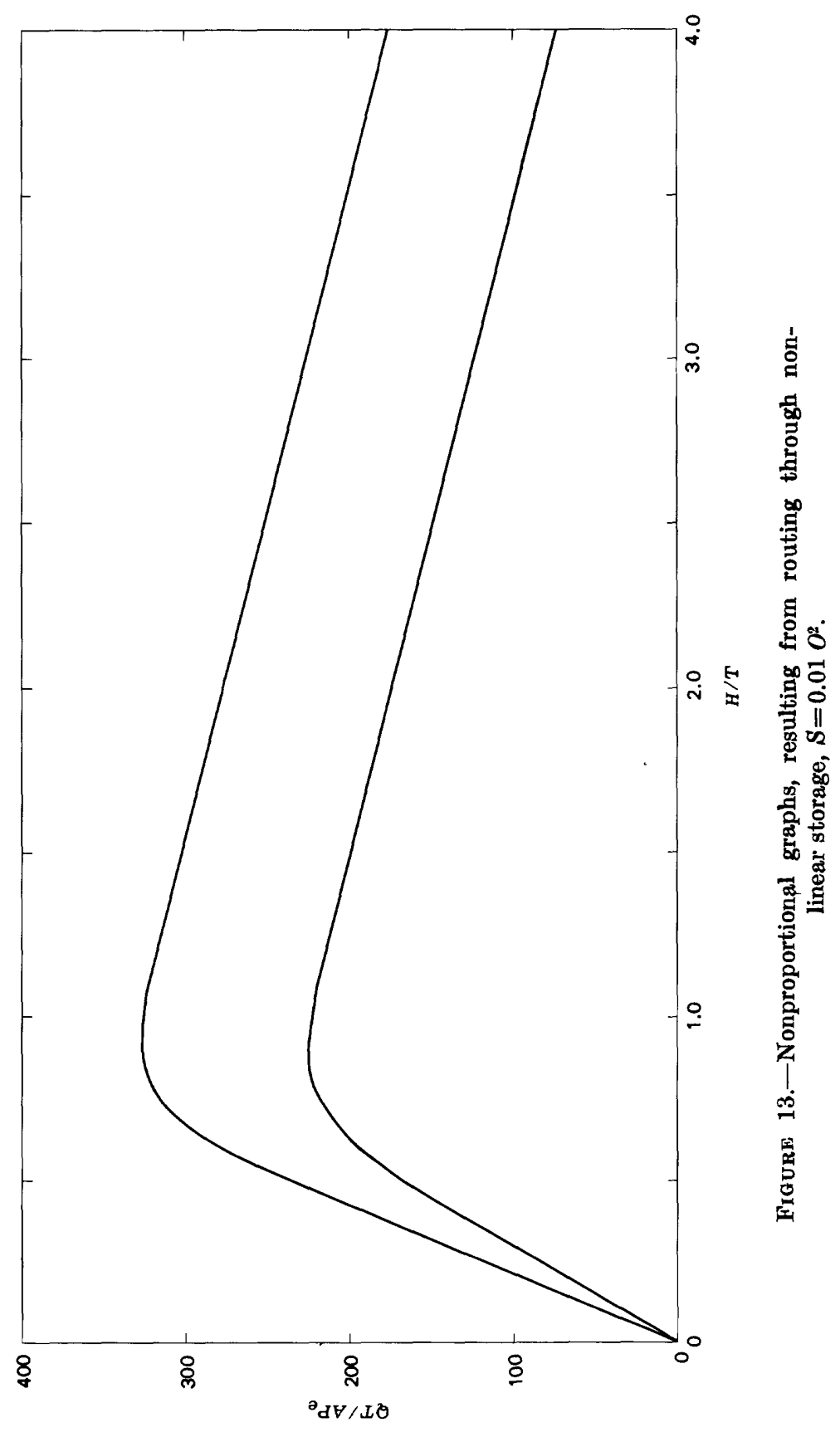




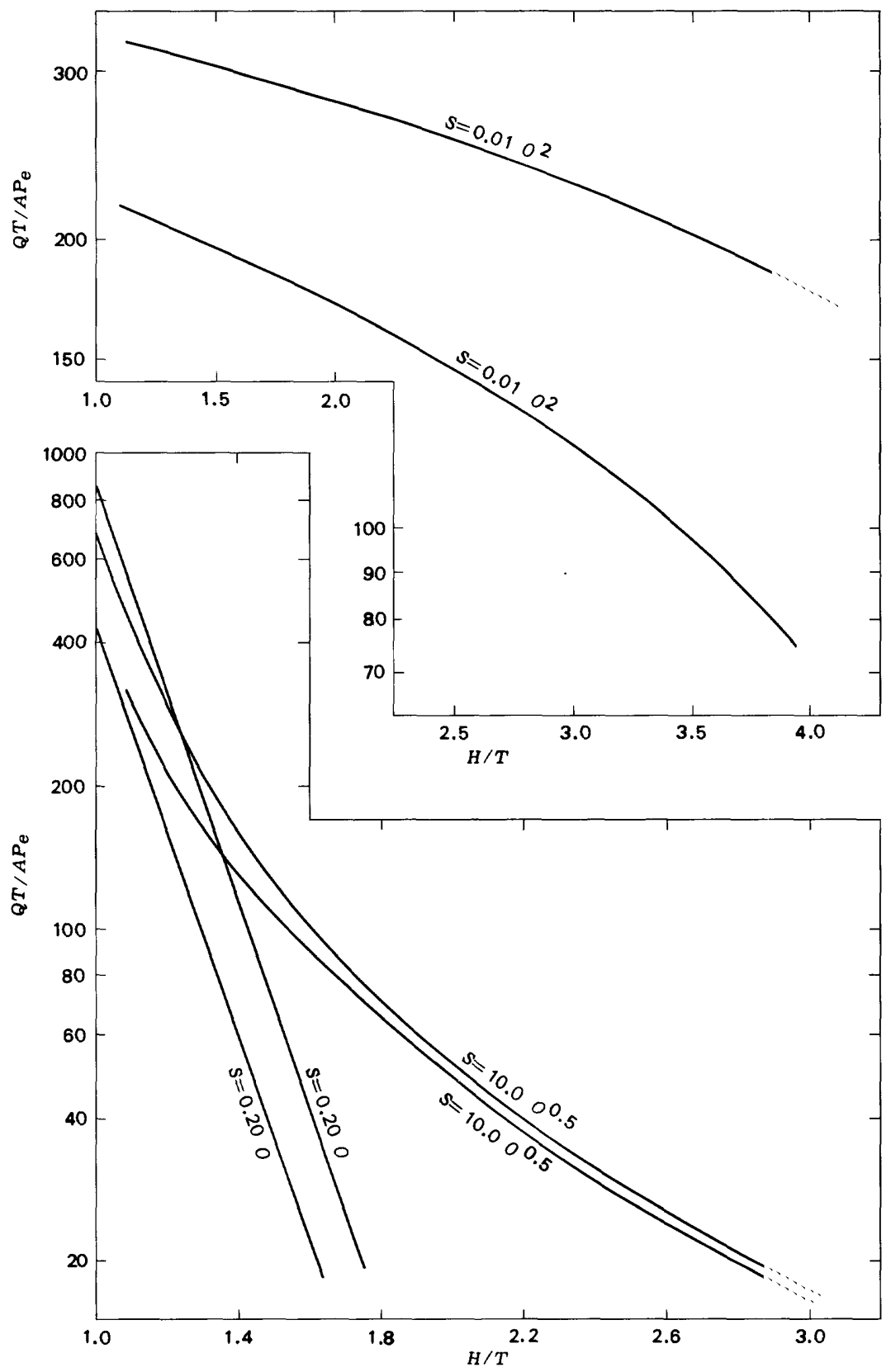

Fiațre 14.-Semilogarithmic plotting, recession limbs of graphs from figures 11, 12 , and 13. 


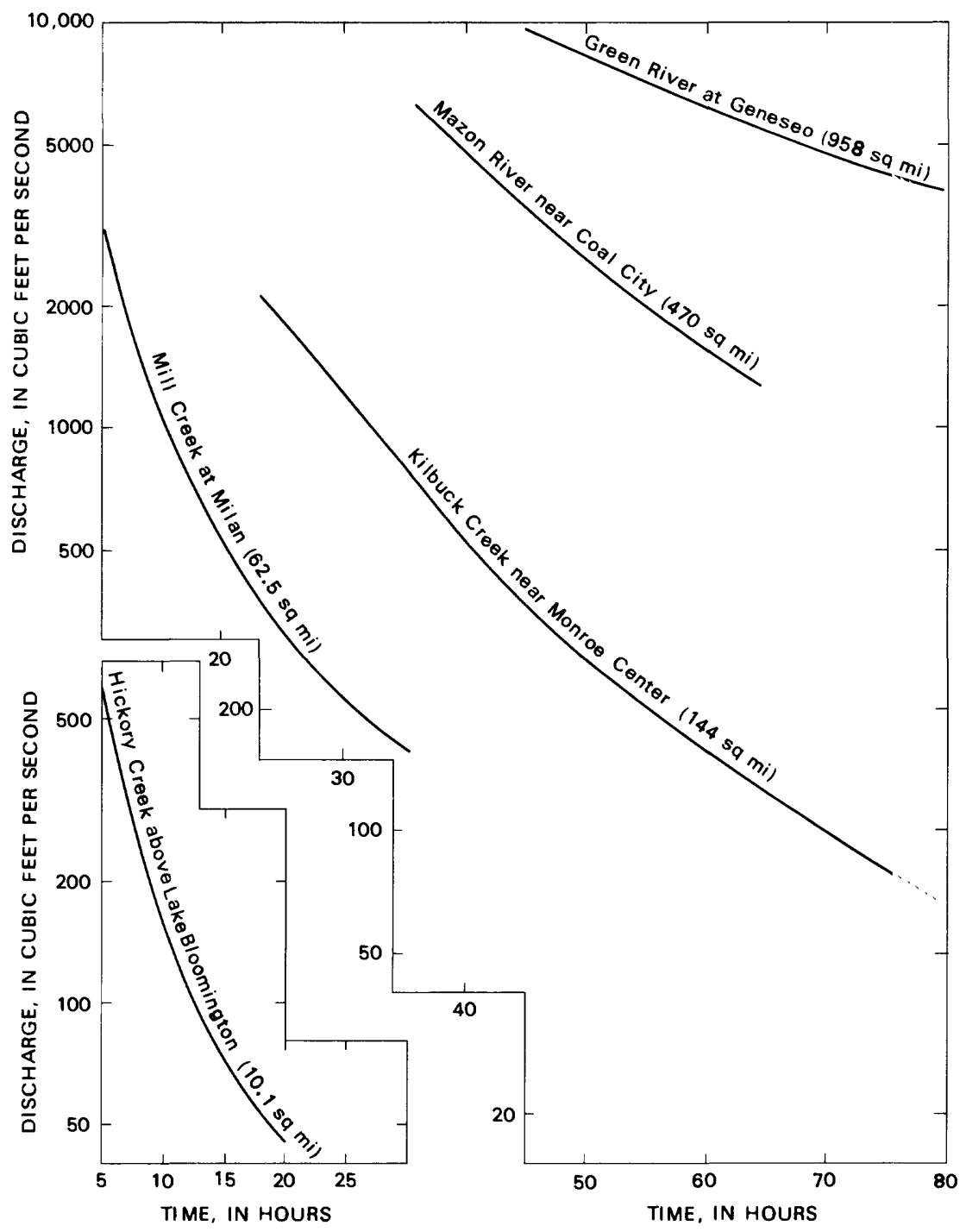

FIGURE 15.-Typical recessions, previously published unit hydrographs.

(Mitchell, 1948) and also the graphs derived by the synthetic methods proposed by Commons (1942), the U.S. Department of Agriculture Soil Conservation Service (1957), and Mitchell (1948). The graph labeled "Mitchell (1962, synthetic)" is the graph derived by methods described earlier in the section "Application to specific areas."

Although some of these last examples have been called unit hydrographs, we should not be swayed by this fact, for the recession lines must be straight in semilogarithmic plots if the storage effect is to be 


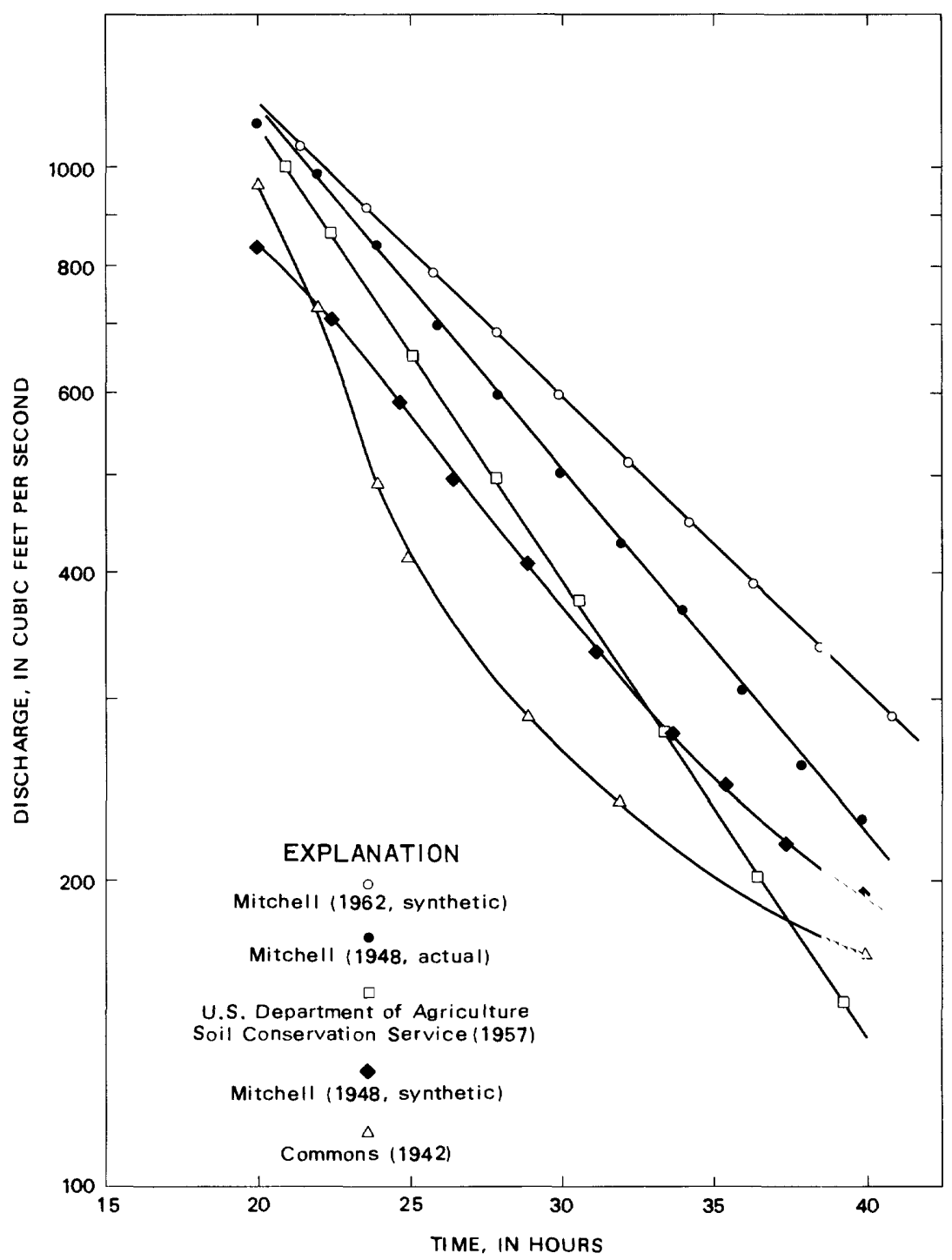

Figure 16.-Recession hydrographs, all for Money Creek above Lak’ Bloomington, Ill., computed by various methods.

considered linear. And the storage effect must be linear if the graphs are to meet the requirement of linear proportionality of ordinates and thus be considered true unit hydrographs.

As mentioned in a foregoing section, areas occasionally are found for which the semilogarithmic plot for the recession limb of the actual hydrograph (even after eliminating base flow) departs significantly from a straight line. True unit hydrographs cannot be developed for 
these areas. Instead, they must be approached with the broader concepts of the model hydrograph.

The special case of the unit hydrograph is applicable only to those areas for which linear storage effect may be assumed. The model hydrograph with its broader concepts is applicable to those areas for which the storage effect is nonlinear.

\section{SUMMARY AND CONCLUSIONS}

Model hydrographs are composed of pairs of dimensionless ratios $\left(H / T, Q T / A P_{e}\right)$ arranged in tabular form. Under appropriate conditions these dimensionless models may be converted to flood hydrographs for specific areas. The conversion is accomplished merely by multiplying the abscissas $(H / T)$ by the value of $T$ for the drainage basin and multiplying the ordinates $\left(Q T / A P_{e}\right)$ by the appropriate value of $A P_{e} / T$.

A dimensionless, isosceles translation hydrograph, for which the time base is $T$ hours, is transformed by summation-curve techniques to a family of hydrographs with storm duration $(D / T)$ ranging from 0.1 to 2.0. Each of these hydrographs is then routed through various single elements of reservoir storage, $S=k O^{x}$ to provide families of model hydrographs. Twenty-six of the families are linear models $(x=1.0)$, with values of $k$ ranging from $0.2 T$ to $3.0 T$. The remaining eight families are nonlinear models, for which $x$ is 0.5 and for which the value of $k / \sqrt{A P_{e} / T}$ ranges from 2.0 to 12.0. The linear models are sufficiently extensive to cover most computational needs; the range of nonlinear models may eventually need to be extended, particularly to cover values of $x$ other than 0.5 and conditions of initial inflow other than zero.

For a specific drainage area, the appropriate model is selected by the following procedure:

1. Determine $T$, in hours, for the basin. If a suitable runoff hydrograph is available, take $T$ as the tlme from ending of rainfall excess to the point of inflection on the recession limb of the hydrograph. If such hydrograph is not available, use the formula

$$
T=5.33 L^{0.602} S_{10 / 85}{ }^{-0.448} S_{t}^{0.231} .
$$

2. Determine values for the storage factors, $k$ and $x$. ( $k$ should be computed in hours; $x$ is a dimensionless number.) If a suitable runoff hydrograph is available, follow the method outlined in the section "Application to Specific Areas" and illustrated by examples $1-5$. If such hydrograph is not avallable, assume $x=5.0$, and compute $k$ by formula

$$
k=17.6 L^{0.339} S_{10 / 85}{ }^{-0.860} S_{\imath}^{0.258} .
$$


(Techniques are not yet available for computing nonlinear storage factors from measured physiographic characteristics.)

3. Determine the storm characteristics, $D$, in hours, and $P_{e}$, in inches. These are obtained from meteorological data and from estimates of precipitation losses, except that when reproducing an observed hydrograph, $P_{e}$ is the volume of surface runoff.

4. Determine the appropriate model family (tables $3-36$ ). If the storage is linear $(x=1.0)$, the family is identified by the value of the ratio $k / T$; if the storage is nonlinear $(x=0.5)$, the family is identified by the value of the ratio $k / \sqrt{A P_{e} / T}$

5. Determine the appropriate member of model family (the column of the table containing proper values of the ordinates $\left.Q T / A P_{e}\right)$. This column is identified by the value of the ratio $D / T$.

The conditions under which the models may be used are often the same as those usually described for unit hydrographs, but with one restriction. In general, the models may be used only to compute the flood hydrograph from storms for which rainfall excess is evenly distributed with respect to both time and area and unaffectec by ice or melting snow. For linear models, the unit-hydrograph techniques of superposition may be used to develop complex hydrographs for storms for which rainfall excess is nonuniform with respect to time. But for nonlinear models superposition techniques are not appropriate. Further, nonlinear models presented here all are predicated on initial flow of zero; more work will be required to determine adjustments necessary when initial flow is substantially greater.

\section{REFERENCES}

Commons, G. G., 1942, Flood hydrographs: Civil Engineering, v. 12, p. 571-572. Mitchell, W. D., 1948, Unit hydrographs in Illinois: Illinois Dept. Public Works and Bldgs. Div. of Waterways, $294 \mathrm{p}$.

-1962, Effect of reservoir storage on peak flow: U.S. Geol. Survey WaterSupply Paper 1580-C, 25 p.

Shen, John, 1962, A Method of determining the storage-outflow characteristics of nonlinear reservoirs: U.S. Geol. Survey Prof. Paper 450-E, p. 167-168.

U.S. Department of Agriculture Soil Conservation Service, 1957, Hydrographs: Natl. Eng. Handb., sec. 4, p. 3.16-1-3.16-14. 
TABLES 3-36

$446-3750-72 \longrightarrow 6$ 


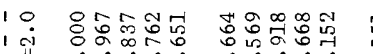

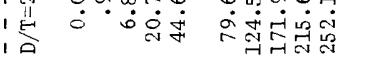

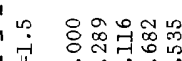

苜

공웡융

윰워요

유뮤

可成落

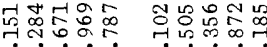

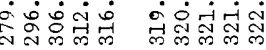

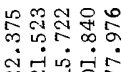

옹

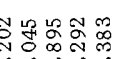

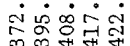

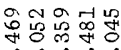

సิ

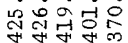

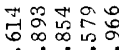

बī

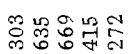

वंबं $\dot{\nabla}$

$\infty \dot{0} \dot{0}$

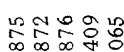

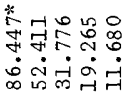

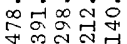

iั

, 8 om

त्रा m

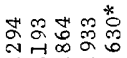

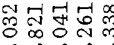

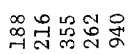

字逭这

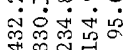

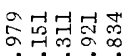

$\therefore$ तิศ

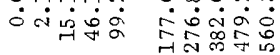

Dront ano *na

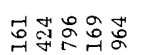

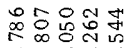

영영엉엥

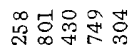

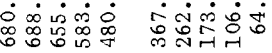

क्षंत्रां

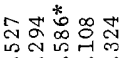

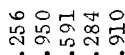

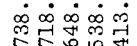

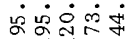

mas

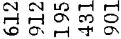

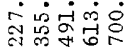

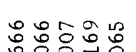

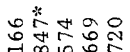

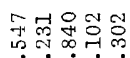

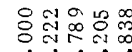

กิ่ง

通岁送券

것홍유

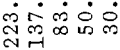

ชั้

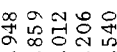

$\dot{0} \dot{0} \sigma \infty \dot{\infty}$

कों

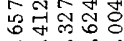

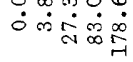

ले

$8 \underset{8}{\infty} \overbrace{\infty}^{\infty} \prod_{\infty}^{\infty}$

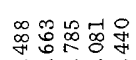

응ㅊㅎㅇㅎㅀ

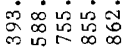

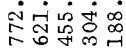

웅유ㅇㅝㅠ

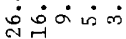

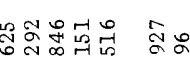

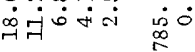

Oें

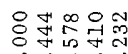

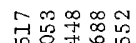

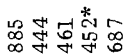

过守实点

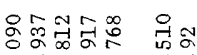

मृंतें

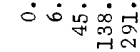

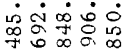

तिं

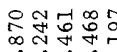

लं $\dot{0} \dot{0} \dot{0}$

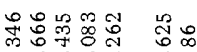

कांमें

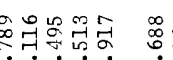

कo 0

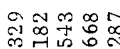

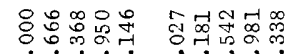

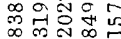

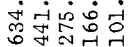

ชิ่

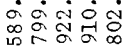

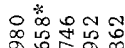

웅요

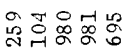

ठ뮹휴웡

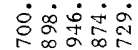

वंतें

พำ

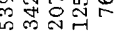

अ

$\dot{0} \dot{+} \dot{ }$

$\dot{\circ} \dot{0}$

뉴요 न

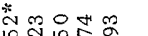

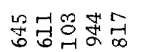

।

, 苟

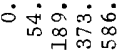

它家灾

ம்

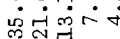

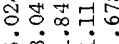

i⿺

๙ूं

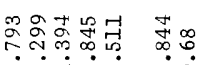

कंत्रा

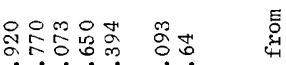

要

$\mid$

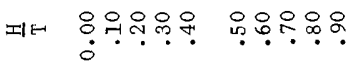

윰윰염

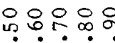

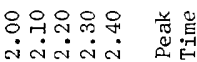

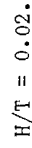


100

I II

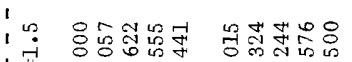

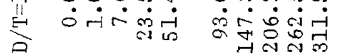

1

10. 8 때

I $\begin{aligned} & \text { म } \\ & \text { I }\end{aligned}$

10

10

1.

12

a$$
\xi
$$

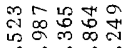

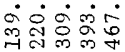
엉융 -

8 도엉묘요 $\dot{0} \dot{-} \dot{4}$

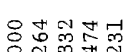

o $0 \dot{0} 0$

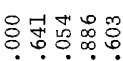

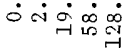

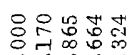

oक 过完

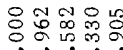

$\dot{0} \dot{0} \dot{\infty} \infty \dot{\infty} \infty$

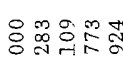

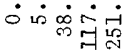

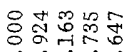

$0 \therefore 50$

$8 \infty$

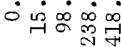

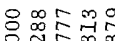

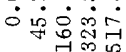

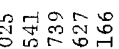

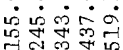

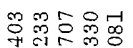

से $\dot{0} \dot{0}$

तथा जा

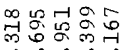

वे ज्ञ

我舫品:

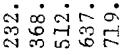

प्रण

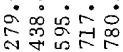

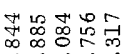

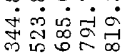

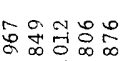

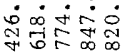

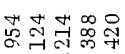

究合命

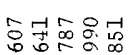

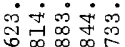

눙요

लंसंबूं

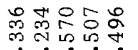

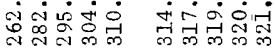

तथ

क

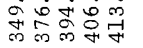

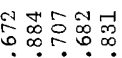

त्र

is

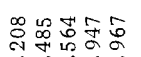

i

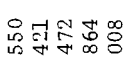

ने

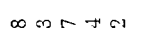

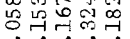

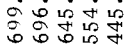

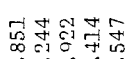

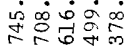

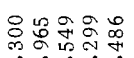

ऽ。

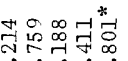

宛官和

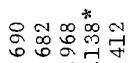

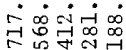

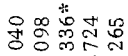

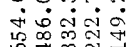

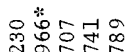

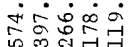

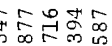

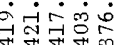

品击宁品

$\forall$
0
0

-1700

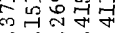

क्षें

엉요

นै.

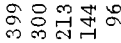

$\infty \infty^{\infty}$

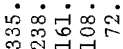

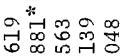

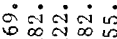

$\forall \forall \forall \infty$

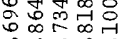

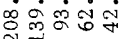

$\therefore$

பु่

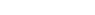

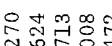

$\dot{\vec{i}} \dot{\infty} \dot{\infty} \dot{\circ} \dot{\infty}$

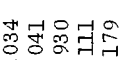

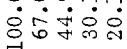

웅됴영

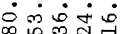

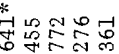

id $\dot{0} \dot{0}$

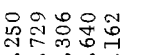

में अं山ें

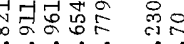

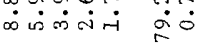

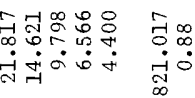

융ํㅕㅇํำ

जित

.

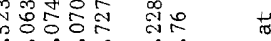

मुनंबंत

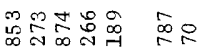

$\dot{0} \dot{x} \dot{0}$

$\infty \dot{\infty}_{\infty}^{\circ}$

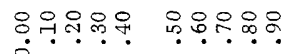

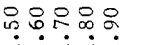

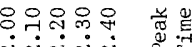
मेंमें 


\begin{tabular}{|c|c|c|c|c|c|c|}
\hline $\begin{array}{l}10 \\
1 \stackrel{0}{\pi} \\
1 \\
1 \\
10\end{array}$ & 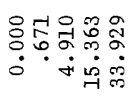 & 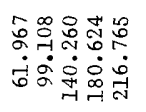 & 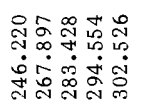 & 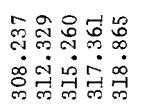 & 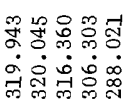 & 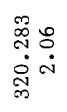 \\
\hline : & 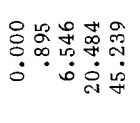 & 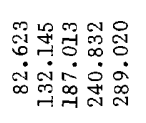 & 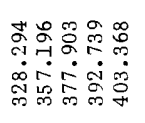 & 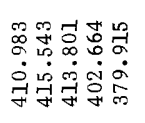 & 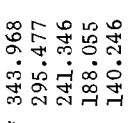 & 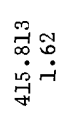 \\
\hline $\begin{array}{l}10 \\
1 \stackrel{11}{1} \\
10\end{array}$ & 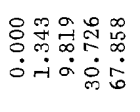 & 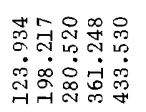 & 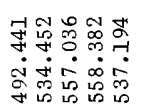 & 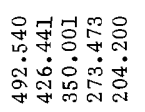 & 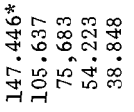 & 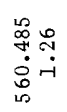 \\
\hline$: \begin{array}{l}0 \\
0 \\
0\end{array}$ & 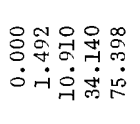 & 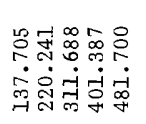 & 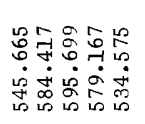 & 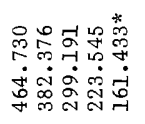 & 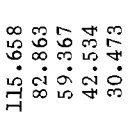 & 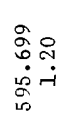 \\
\hline $\begin{array}{l}\infty \\
1 \\
11 \\
1 \\
1 \\
1\end{array}$ & 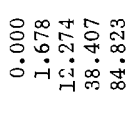 & 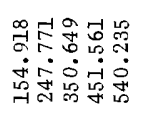 & 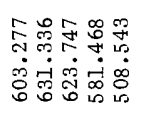 & 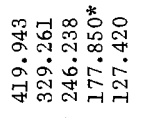 & 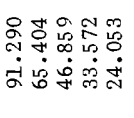 & 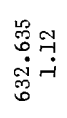 \\
\hline : & 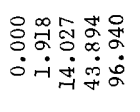 & 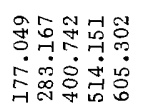 & 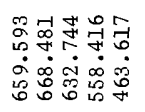 & 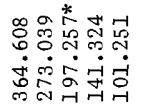 & 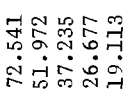 & 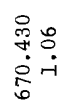 \\
\hline 遂 & 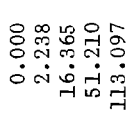 & 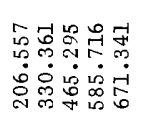 & 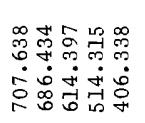 & 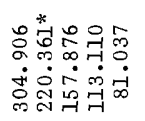 & 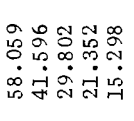 & 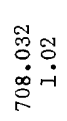 \\
\hline : & 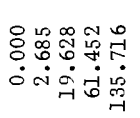 & 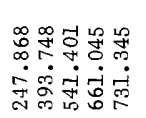 & 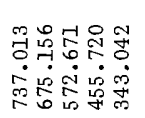 & 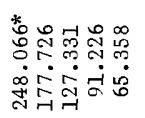 & 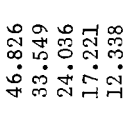 & 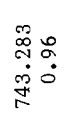 \\
\hline $\begin{array}{l}1 \\
\vdots \\
10 \\
1 \\
1 \\
10\end{array}$ & 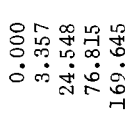 & 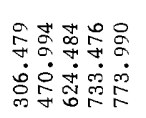 & 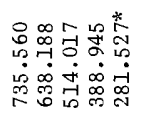 & 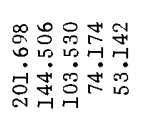 & 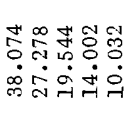 & 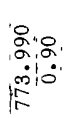 \\
\hline : & 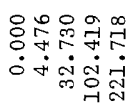 & 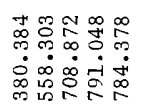 & 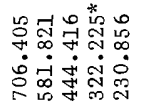 & 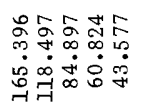 & 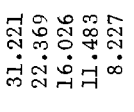 & 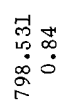 \\
\hline $\begin{array}{l}10 \\
10 \\
10 \\
10 \\
10\end{array}$ & 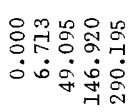 & 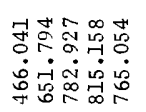 & 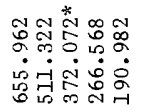 & 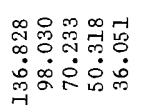 & 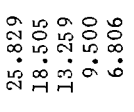 & 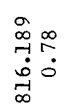 \\
\hline :-1 & 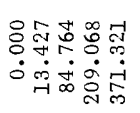 & 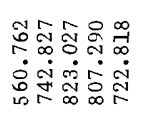 & 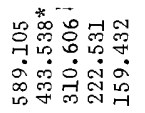 & 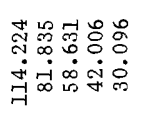 & 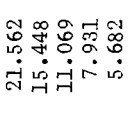 & 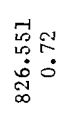 \\
\hline 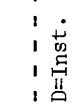 & 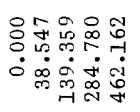 & 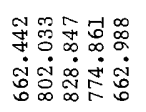 & 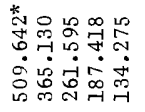 & 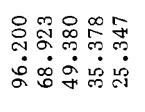 & 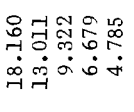 & 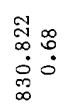 \\
\hline$\Rightarrow$ 데터 & 웅규유요유 & 윰융ㅇㅇㅇㅛ. & 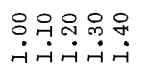 & 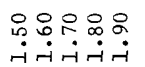 & 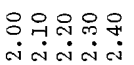 & 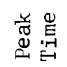 \\
\hline
\end{tabular}




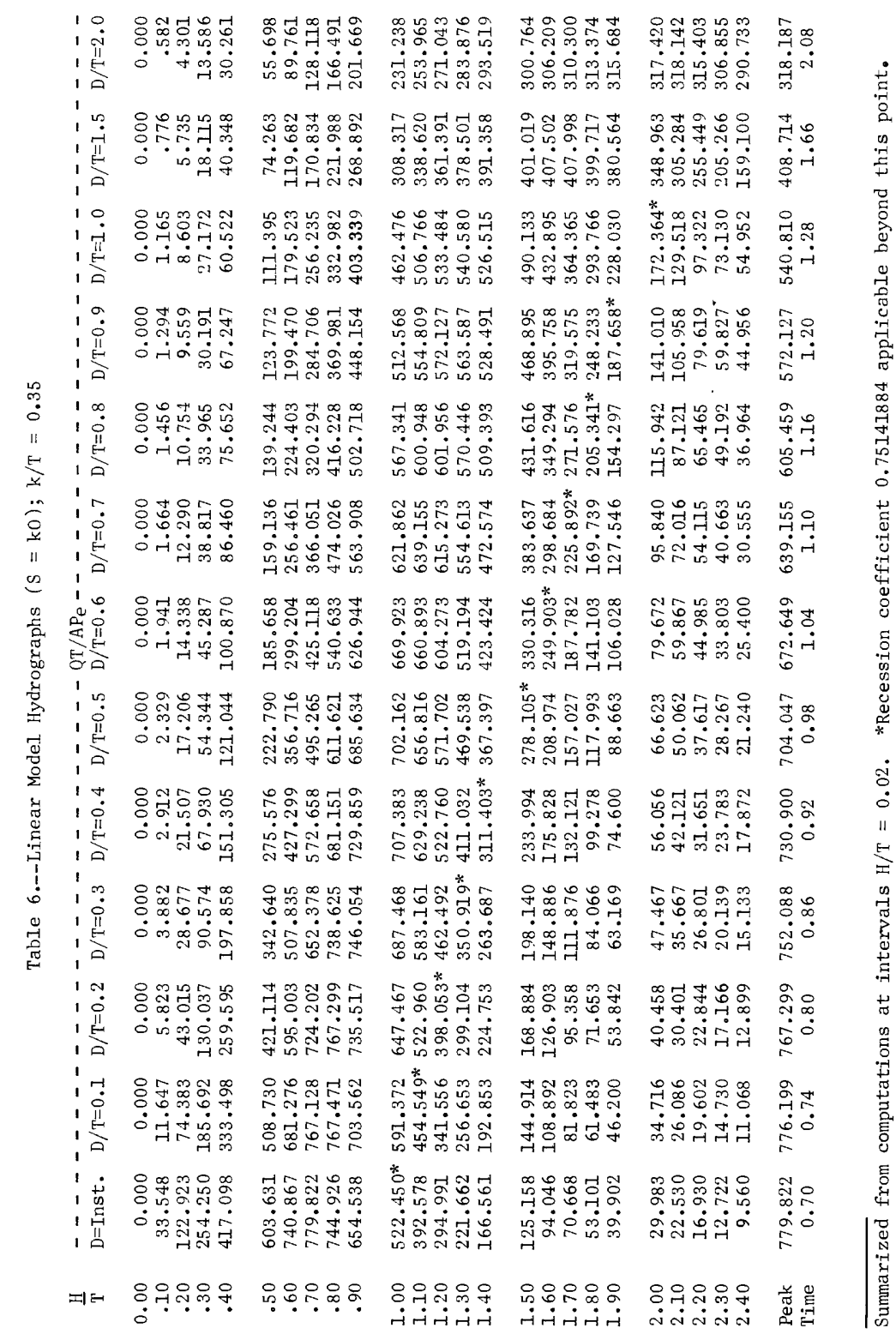




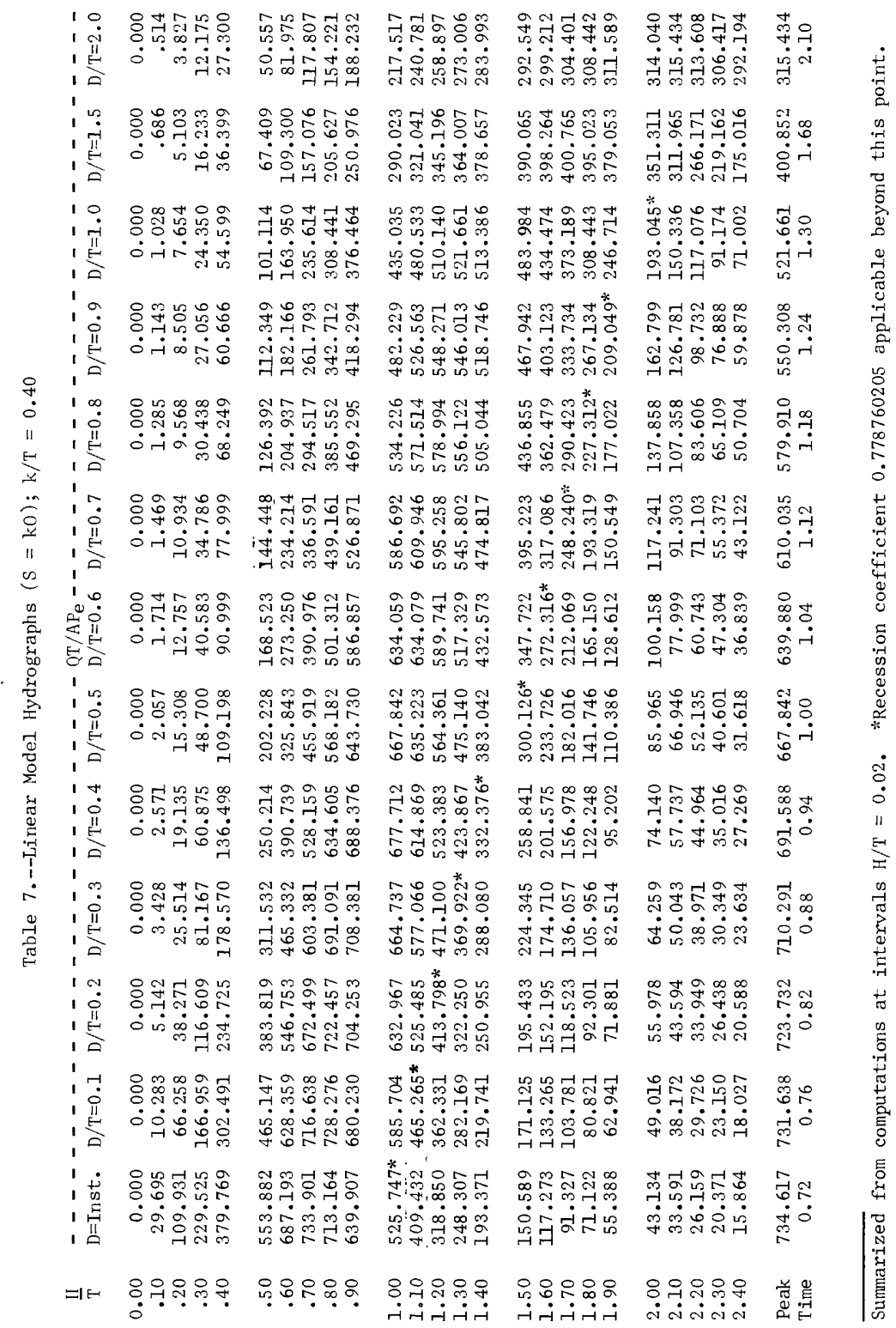




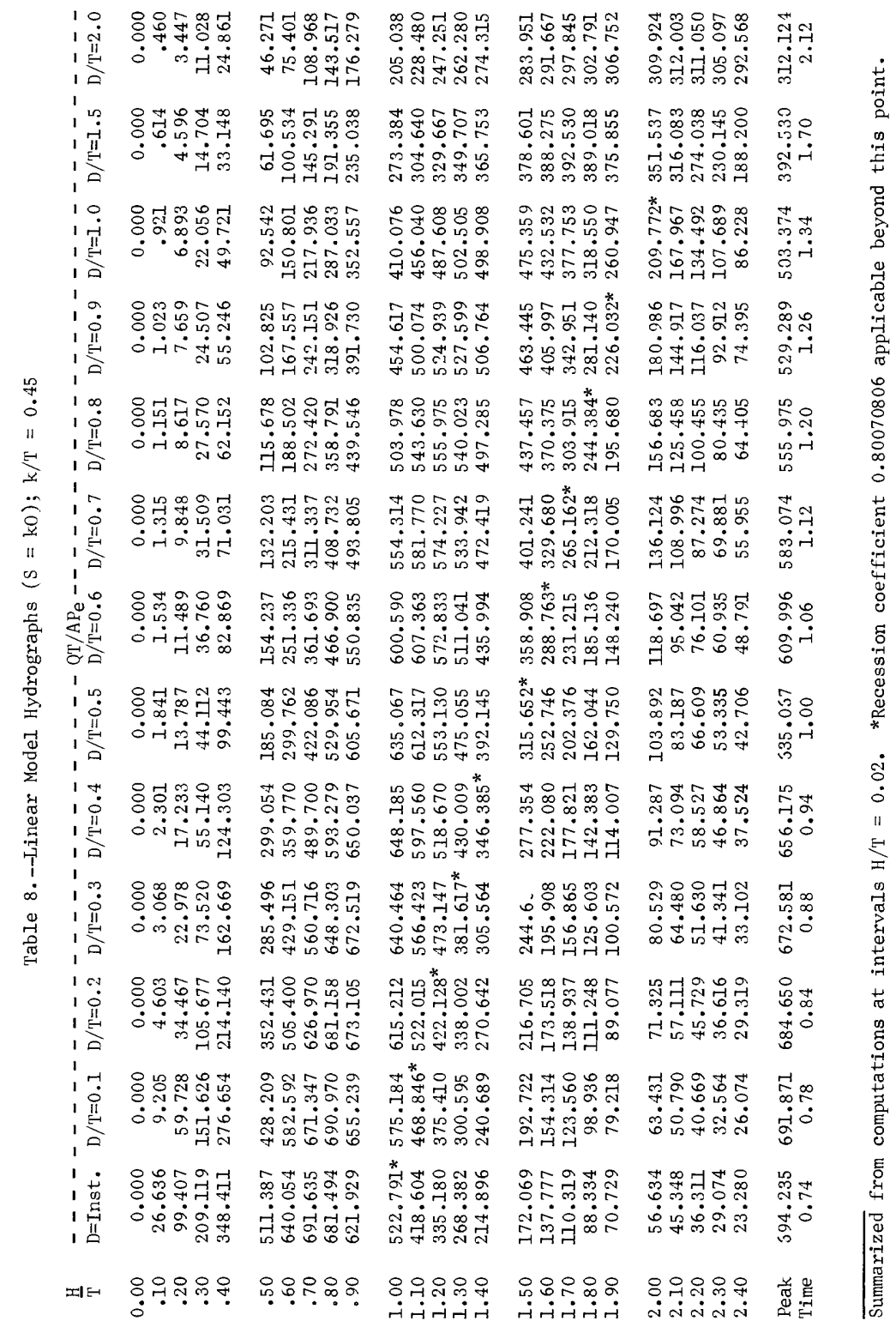




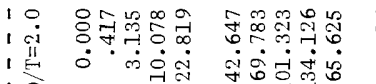

10

1 เ

1 II

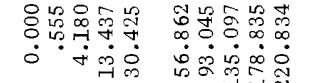

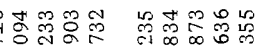

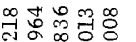

इनें

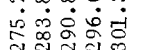

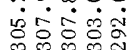

$\infty$
$\infty$
$\infty$
$\infty$

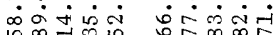

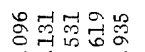

영

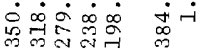

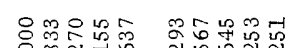

min 00

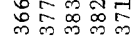

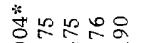

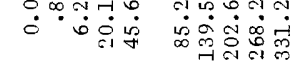

भ.

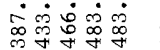

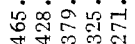

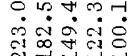

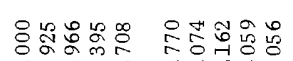

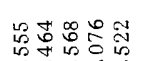

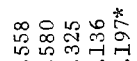

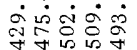

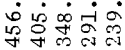

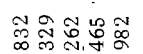

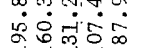

赵.

$\sum_{0}^{\infty} \sim$

$\dot{0} \cdot \dot{0}$ 夜

A1 $-1-4-4$

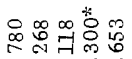

$\dot{\circ} \dot{i} \dot{\varphi} \dot{0}$

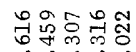

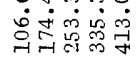

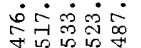

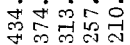

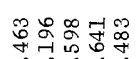

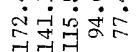

象

\&ें

$\stackrel{\infty}{\stackrel{\infty}{\sim}}$

क

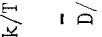

के i

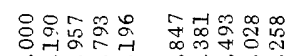

$\dot{0}+\dot{\infty} \infty \dot{\infty}$

तᄀ่

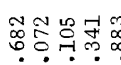

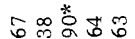

암쪅영

: ํํㅇ

मृं

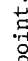

2

范

응영ำ

○ं

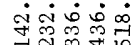

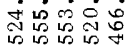

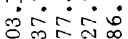

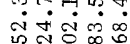

$\stackrel{\infty}{\rightarrow+5}$

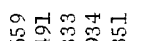

붕에

oOn

i⿱⺈

वृ

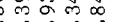

:

$7+\frac{7}{7}$

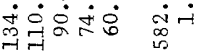

워ㄱㅝㅓㅇ웜

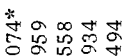

造骂灾然

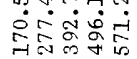

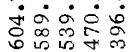

$\dot{\theta} \dot{0} \dot{\infty} \dot{0}$

대요

नी

சி

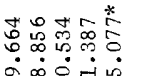

ํํㅇำ

ㄱำ

육웛

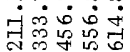

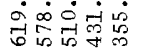

वें

b

$\infty$

¿े

i

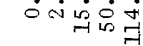

蜍票吉

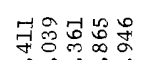

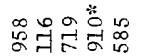

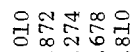

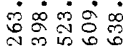

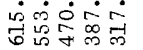

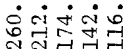

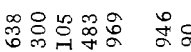

요욤

여ํำำ

용

aoㄴ

หูं

ปิำ

我

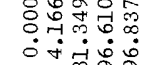

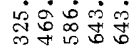

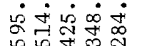

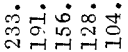

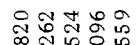

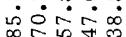

정헤

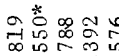

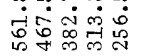

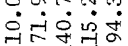

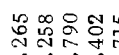

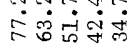

5

迥

*두요

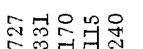

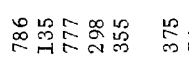

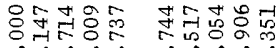

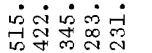

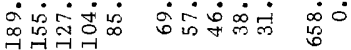

8웍웍윰유

운융ㅇㅇㅇ

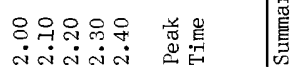




\begin{tabular}{|c|c|c|c|c|c|c|}
\hline $\begin{array}{l}10 \\
1 \\
11 \\
10 \\
1\end{array}$ & 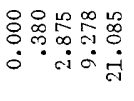 & 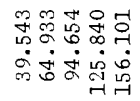 & 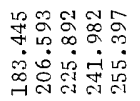 & $\begin{array}{l}1 \\
0\end{array}$ & 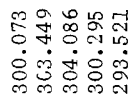 & 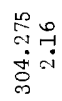 \\
\hline $\begin{array}{l}1 \\
10 \\
1 \\
11 \\
10\end{array}$ & 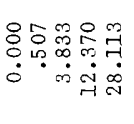 & 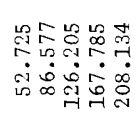 & 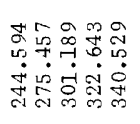 & 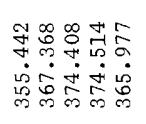 & 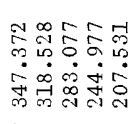 & 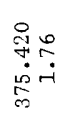 \\
\hline \begin{tabular}{ll}
1 & 0 \\
1 & -1 \\
1 & \multicolumn{1}{|c}{} \\
1 & 0
\end{tabular} & 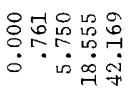 & 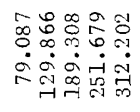 & 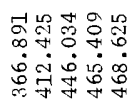 & 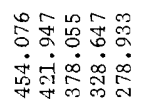 & 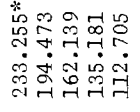 & 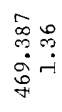 \\
\hline $\begin{array}{l}1 \\
1 \\
1 \\
1 \\
1 \\
1\end{array}$ & 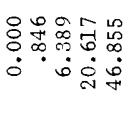 & 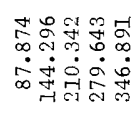 & 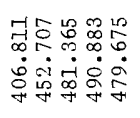 & 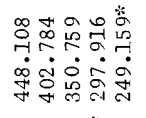 & 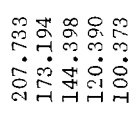 & $\begin{array}{l}\infty \\
\infty \\
\infty \\
\vdots \\
0 \\
\infty \\
\sigma\end{array}$ \\
\hline $\begin{array}{l}1 \infty \\
1 \\
1 \\
1 \\
1 \\
1\end{array}$ & 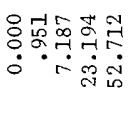 & 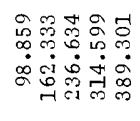 & 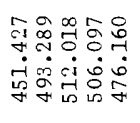 & 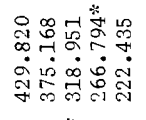 & 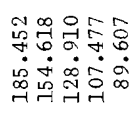 & $\begin{array}{ll}0 \\
0 \\
\infty \\
\infty \\
01 \\
-1 \\
0\end{array}$ \\
\hline 10 & 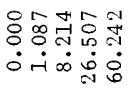 & 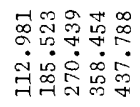 & 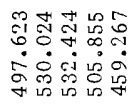 & 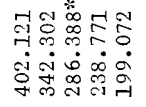 & 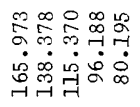 & 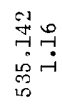 \\
\hline 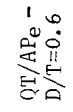 & 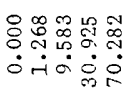 & 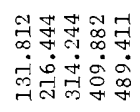 & 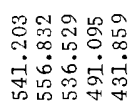 & 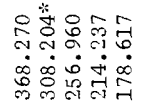 & 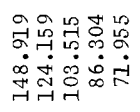 & 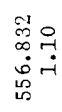 \\
\hline $\begin{array}{l}1 \\
1 \\
1 \\
1 \\
1 \\
1\end{array}$ & 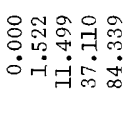 & 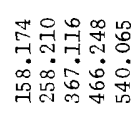 & 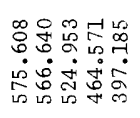 & 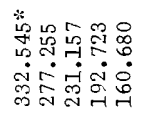 & 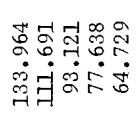 & 学 \\
\hline $\begin{array}{l}1 \\
1 \\
1 \\
1 \\
10 \\
10\end{array}$ & 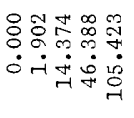 & 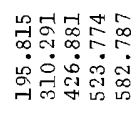 & 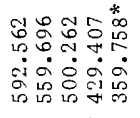 & 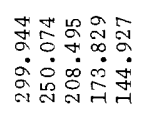 & 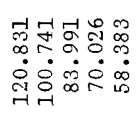 & 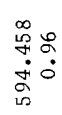 \\
\hline : & 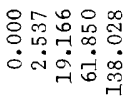 & 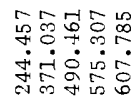 & 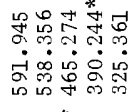 & 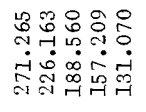 & 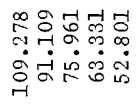 & 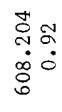 \\
\hline $\begin{array}{ll}1 & 0 \\
1 & 0 \\
1 & 11 \\
1 & 0\end{array}$ & 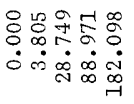 & 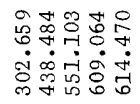 & 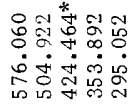 & 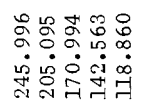 & 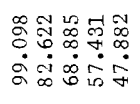 & $\begin{array}{l}0 \\
\tilde{o}^{\circ} \\
\infty \\
0 \\
0 \\
0\end{array}$ \\
\hline $\begin{array}{l}17 \\
1 \\
1 \\
11 \\
0\end{array}$ & 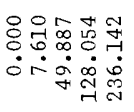 & 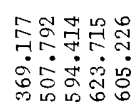 & 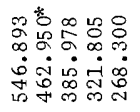 & 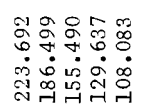 & 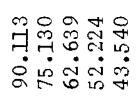 & 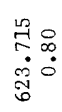 \\
\hline $\begin{array}{ll}1 & \dot{4} \\
1 & 0 \\
1 & 0 \\
1 & 0\end{array}$ & 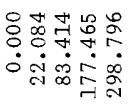 & 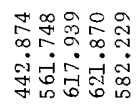 & 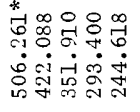 & 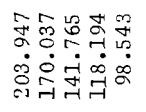 & $\begin{array}{l}\infty \\
\infty\end{array}$ & 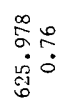 \\
\hline : & \&윰유윰 & 욤욤요. & & 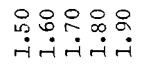 & 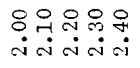 & 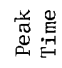 \\
\hline
\end{tabular}




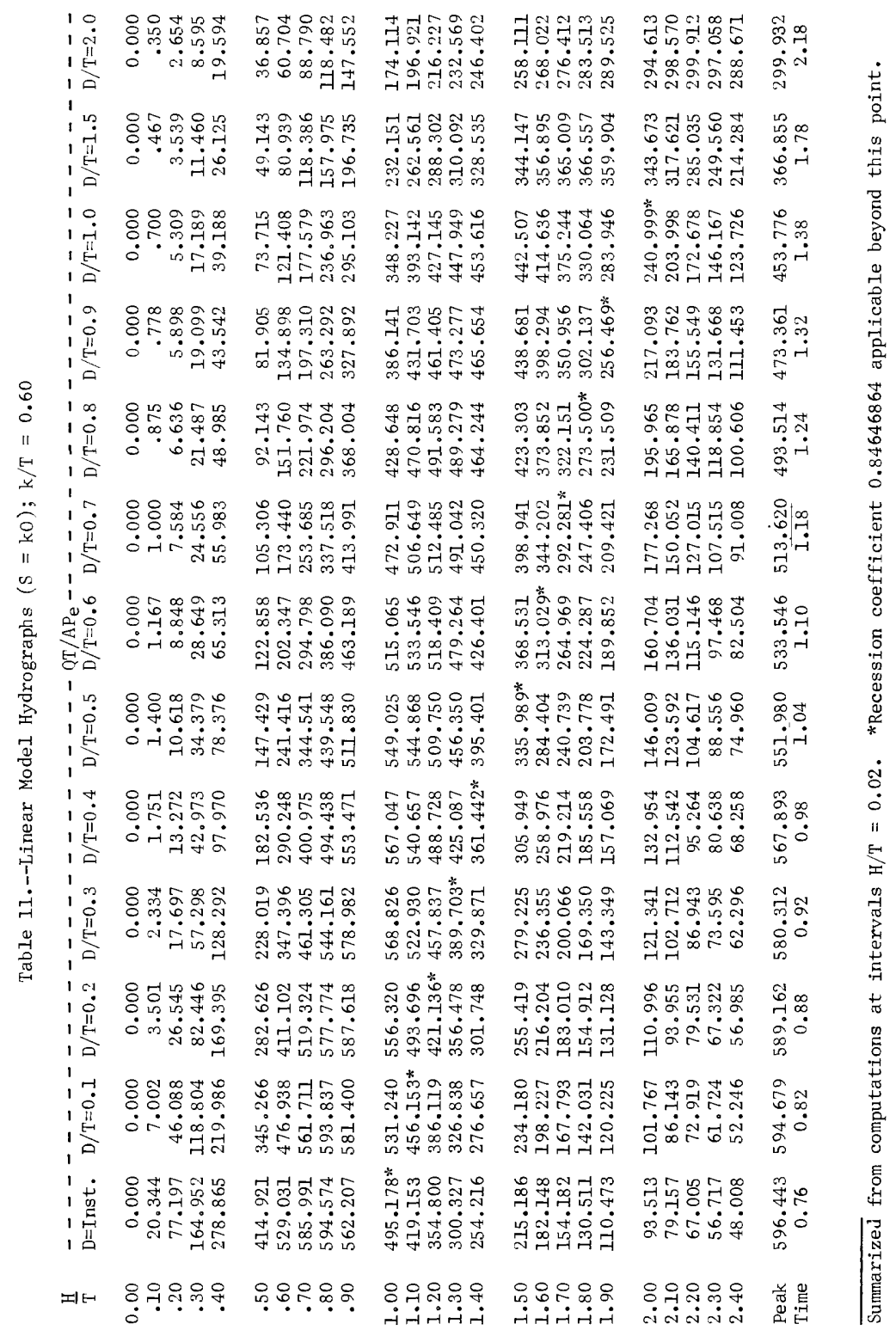




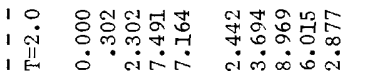

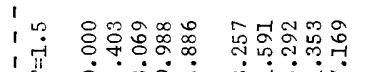

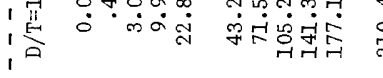

1

I

।

1 II

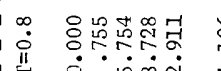
तิ

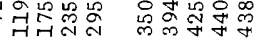

ㄴ.

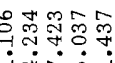

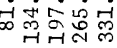

各尽李怘点

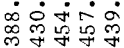

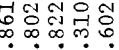

जิํำ

윔융융윰

寉客客:

융궁동요

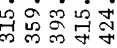

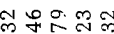

円亦凩

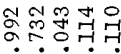

걱웡웡

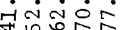

مि

$0 \pi 4 m$

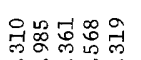

సं

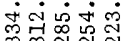

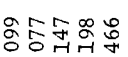

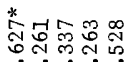

ᄀᄀํำ

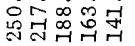

구우운

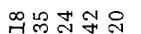

영

구음

न

จิळisa

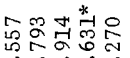

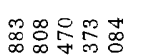

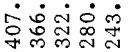

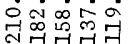

ำ

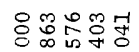

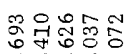

겅옹융유

०ं

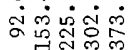

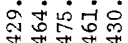

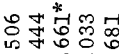

N几 $-2-1$

워

:

品-

옹

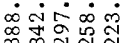

西

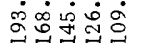

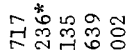

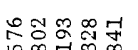

익ㅇㅇㅇㅇㅇ

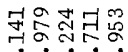

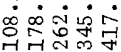

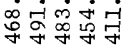

$\dot{0} \dot{0} \dot{0}$

เ๐

ठำ

사잉

두궁 लॉल

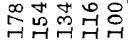

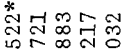

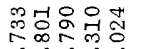

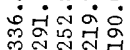

சீं

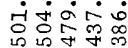

ิㅗㄱ휴웡

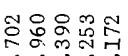

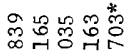

a N

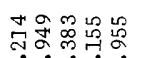

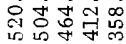

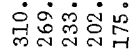

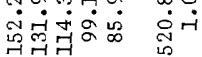

苨

$\rightarrow$

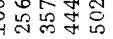

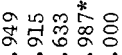

में

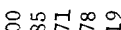

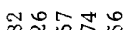

茫

家家家过

혐ㅁㅇㅇㅎㅇ

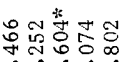

$\dot{0} \dot{0} \dot{0} \dot{0}$

청ㅇำ

$\dot{\circ} \dot{0} \dot{0} \dot{0}$

this

$\infty \circ$

I

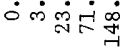

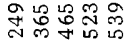

武东宗贫命

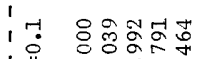

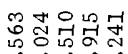

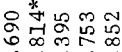

oraa

ไิ่งกำ

$\dot{0} \dot{0} \dot{0} \dot{\infty} \dot{\infty} \dot{\infty} \dot{0}$

$+$

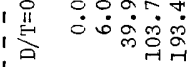

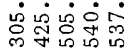

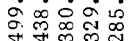

잉

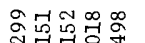

安迆

-

(

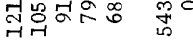

।

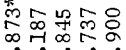

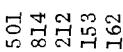

끙ㅇำ

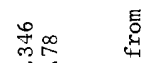

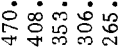

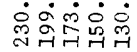

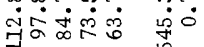

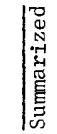

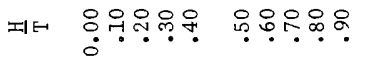

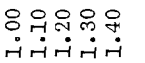

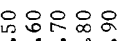

응유윰유

密罢

ธ่

王 


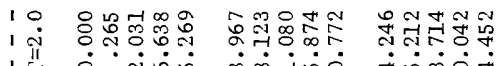

10

is

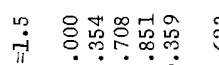

点

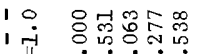

1 in

10

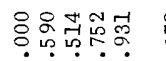

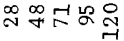

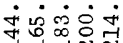

요의

$\sim \Re \infty \infty$

m.

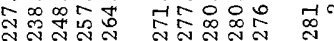

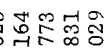

宓实完

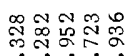

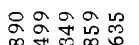

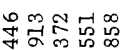

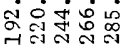

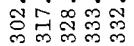

क्ष

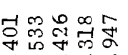

* $m$ on

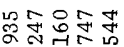

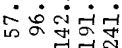

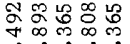

i̊

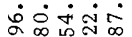

$0.0 \%$

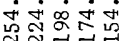

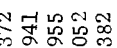

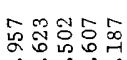

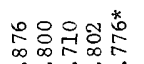

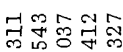

नें

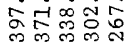

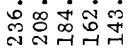

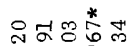

융요

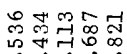

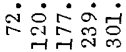

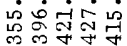

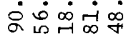

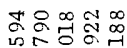

जें

लॉल

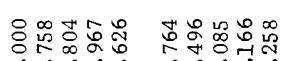

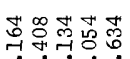

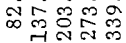

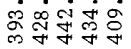

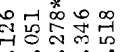

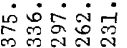

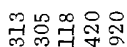

过家宑完离

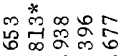

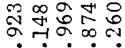

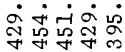

我这完

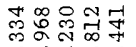

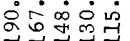

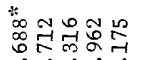

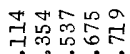

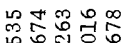

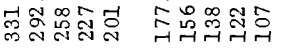

$\therefore \dot{\infty} \dot{0}$

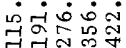

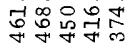

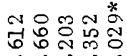

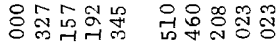

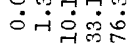

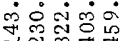

官京家客昆

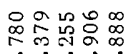

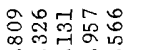

จุं

मㅎํ용

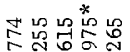

갱병영용?

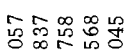

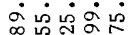

ம்

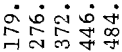

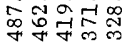

跡元元

(1)

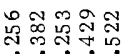

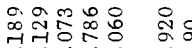

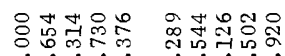

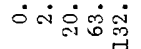

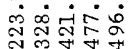

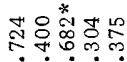

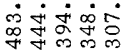

संष्तें

अं

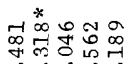

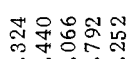

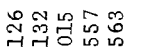

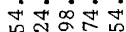

$\dot{0} \dot{0} \infty$

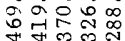

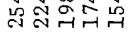

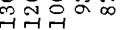

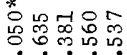

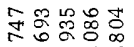

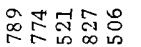

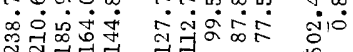

Fे

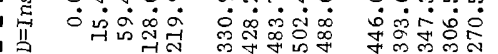

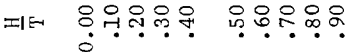

욱워ㅁㅠㅠㅁํํ

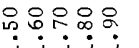

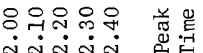

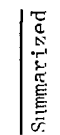




\begin{tabular}{|c|c|c|c|c|c|c|}
\hline $\begin{array}{ll}1 & 0 \\
1 & 0 \\
1 & i \\
1 & 1 \\
1 & 0\end{array}$ & 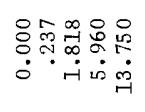 & 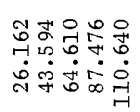 & 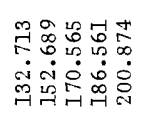 & 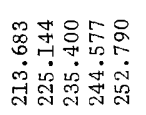 & 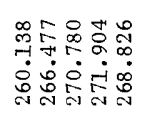 & 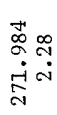 \\
\hline 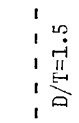 & 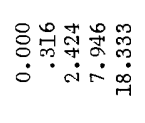 & 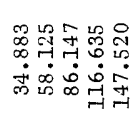 & 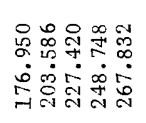 & 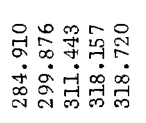 & 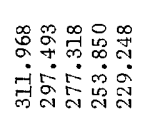 & 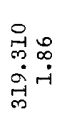 \\
\hline $\begin{array}{l}10 \\
1 \\
1 \\
11 \\
10\end{array}$ & 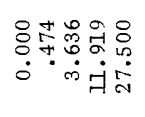 & 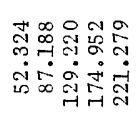 & 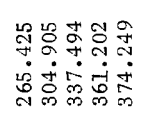 & 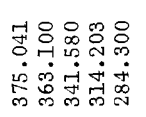 & 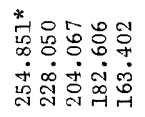 & 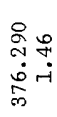 \\
\hline $\begin{array}{l}1 \\
\vdots \\
1 \\
0 \\
10 \\
0\end{array}$ & 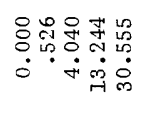 & 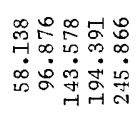 & 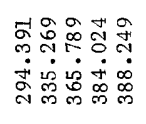 & 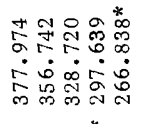 & 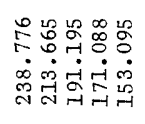 & 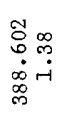 \\
\hline $\begin{array}{l}1 \stackrel{\infty}{\infty} \\
1 \stackrel{0}{\mu} \\
1 \\
1\end{array}$ & 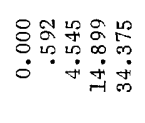 & 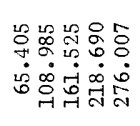 & 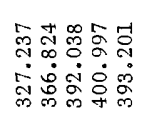 & 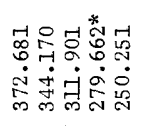 & 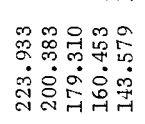 & 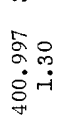 \\
\hline $\begin{array}{l}1 \\
\dot{0} \\
1\end{array}$ & 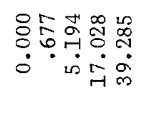 & 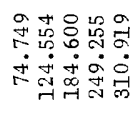 & 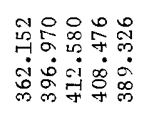 & 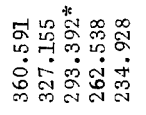 & 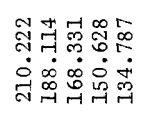 & 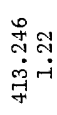 \\
\hline 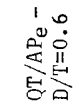 & 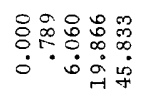 & 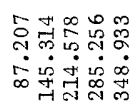 & 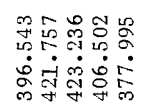 & 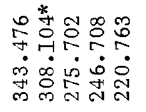 & 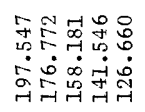 & 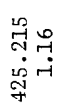 \\
\hline $\begin{array}{ll}1 & 0 \\
1 & 0 \\
10 \\
1 \\
1\end{array}$ & 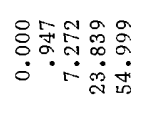 & 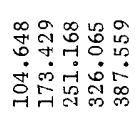 & 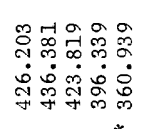 & 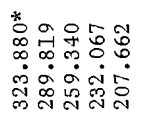 & 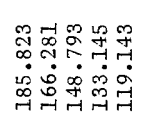 & \\
\hline $\begin{array}{l}1 \stackrel{40}{1} \\
10 \\
1 \\
1\end{array}$ & 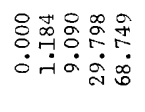 & 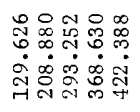 & 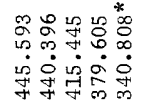 & 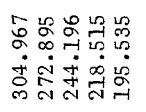 & 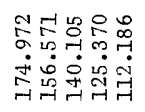 & 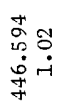 \\
\hline $\begin{array}{l}\infty \\
0 \\
0 \\
11 \\
1 \\
1\end{array}$ & 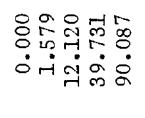 & 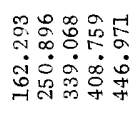 & 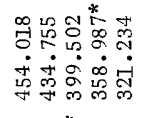 & 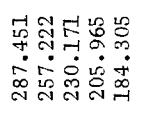 & 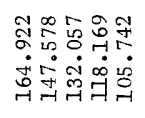 & 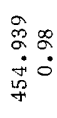 \\
\hline $\begin{array}{ll}1 & 0 \\
1 & 0 \\
1 & 11 \\
1 & 0\end{array}$ & 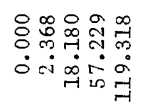 & 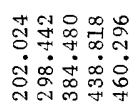 & 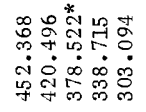 & 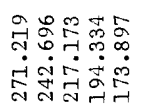 & 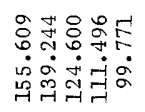 & $\begin{array}{l}\text { केषे } \\
\text { कें } \\
\text { के }\end{array}$ \\
\hline $\begin{array}{ll}1 & 1 \\
1 & 0 \\
1 & 11 \\
1 & 0 \\
1 & 0\end{array}$ & 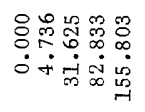 & 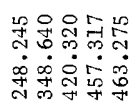 & 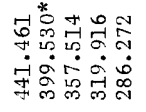 & 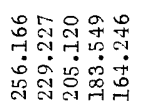 & 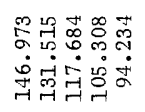 & 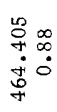 \\
\hline $\begin{array}{l}1 \\
1 \\
1 \\
1 \\
\text { 苔 } \\
\text { 范 }\end{array}$ & 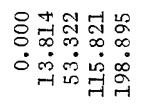 & 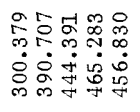 & 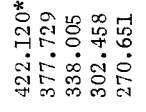 & 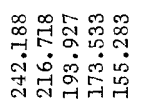 & 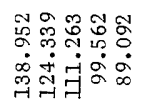 & $\begin{array}{l}0 \\
\infty \\
\infty \\
\dot{0} \\
0 \\
\forall\end{array}$ \\
\hline : 되 & 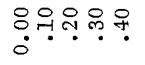 & 운웅용요. & 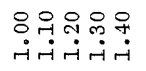 & 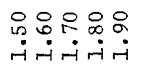 & 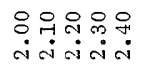 & 量 \\
\hline
\end{tabular}




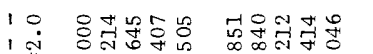

I II

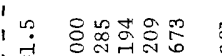

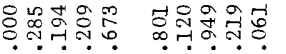

ले

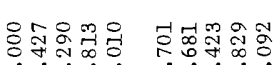

$\dot{0} \dot{0} \dot{0}$

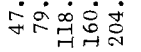

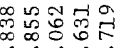

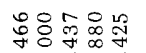

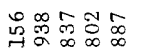

तิ่

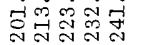

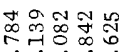

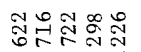

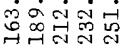

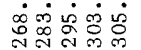

다에

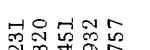

我离离离品

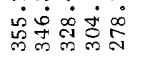

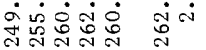

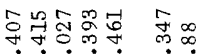

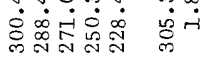

*

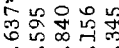

मैं

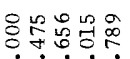

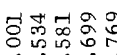

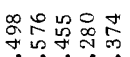

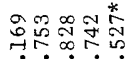

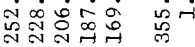

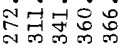

نें字客

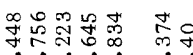

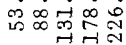

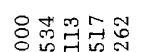

청훙주유

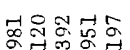

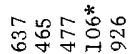

$\dot{0} \dot{0} \dot{0} \dot{0} \dot{0}$

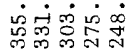

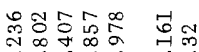

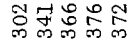

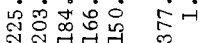

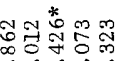

옹ㅁ⿱ㅇㅇ윰옹

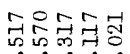

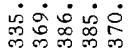

अंते

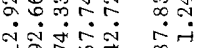

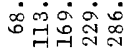

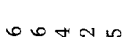

菏 $\rightarrow$ ก

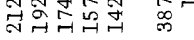

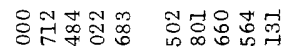

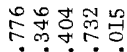

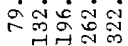

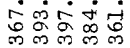

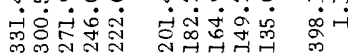

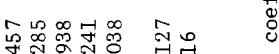

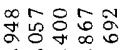

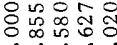

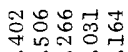

* $10 m$

$8: 010$

की

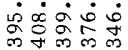

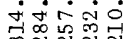

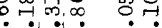

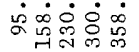

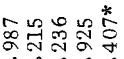

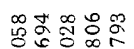

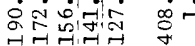

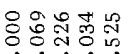

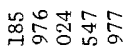

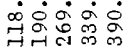

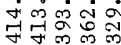

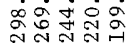

ำำำำำ

8

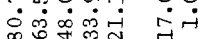

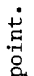

10

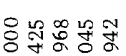

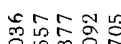

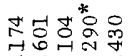

$\dot{\circ} \dot{0} \dot{0} \dot{0} \dot{\infty}$

过守灾票

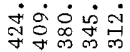

동요ㅇㅝㅕ영

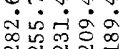

$\forall$

苗

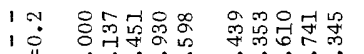

I II

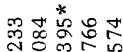

앴

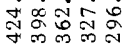

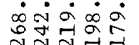

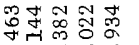

सं山्व்

$\therefore \%$

1)

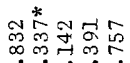

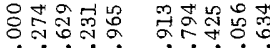

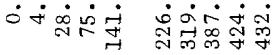

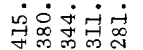

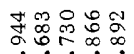

म요 $\dot{0} \infty$

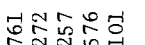

กลสำ

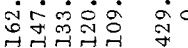

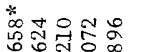

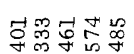

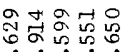

过家宗密

-1
$\sigma_{0}^{\infty}$
$\dot{0} 0$

䑻

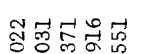

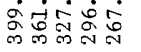

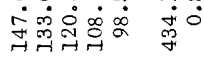

岇苾 进

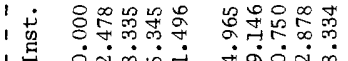
i०ळoi

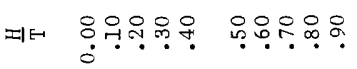

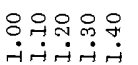

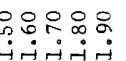

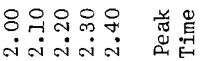

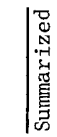




\begin{tabular}{|c|c|c|c|c|c|c|}
\hline $\begin{array}{l}10 \\
1 \\
1 \\
1 \\
1 \\
1\end{array}$ & 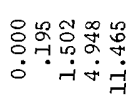 & 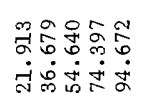 & 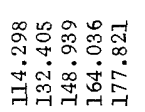 & 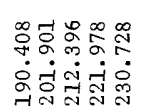 & 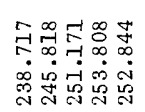 & 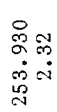 \\
\hline $\begin{array}{ll}1 & 0 \\
1 & 0 \\
10 & 0 \\
1 & 0\end{array}$ & 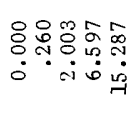 & 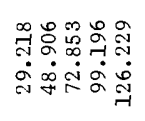 & 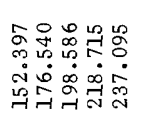 & 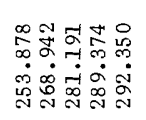 & 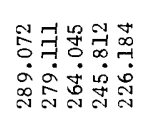 & 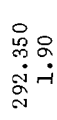 \\
\hline $\begin{array}{l}10 \\
10 \\
10 \\
10\end{array}$ & 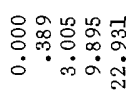 & 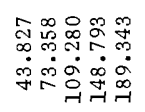 & 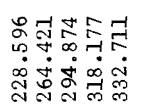 & 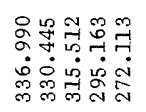 & 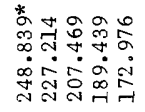 & 离 \\
\hline $\begin{array}{l}a \\
0 \\
11 \\
10\end{array}$ & 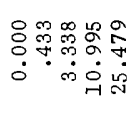 & 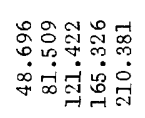 & 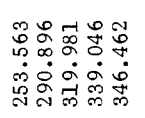 & 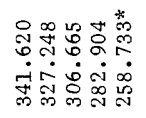 & 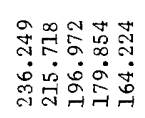 & 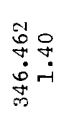 \\
\hline $\begin{array}{l}1 \infty \\
1 \\
11 \\
1 \\
1 \\
1\end{array}$ & 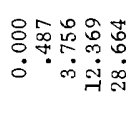 & 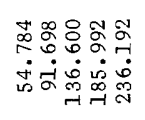 & 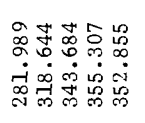 & 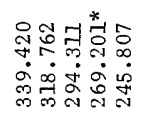 & 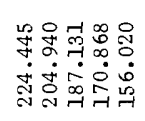 & $\begin{array}{l}2 \\
\infty \\
\infty \\
\infty \\
\infty \\
\infty \\
\infty\end{array}$ \\
\hline : & 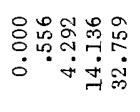 & 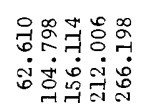 & 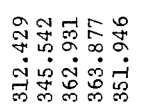 & 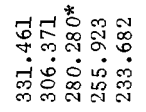 & 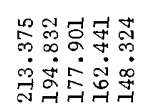 & 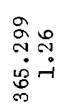 \\
\hline 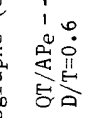 & 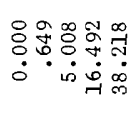 & 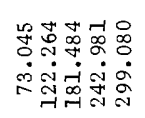 & 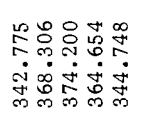 & 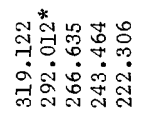 & 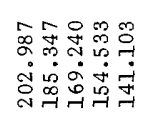 & 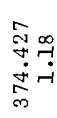 \\
\hline $\begin{array}{l}1 \\
0 \\
0 \\
110 \\
1 \\
1 \\
1\end{array}$ & 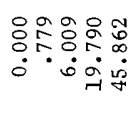 & 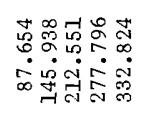 & 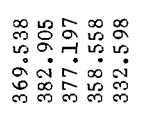 & 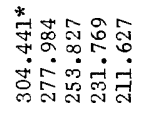 & 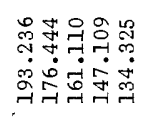 & 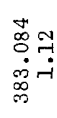 \\
\hline $\begin{array}{l}1 \\
1 \\
1 \\
11 \\
10 \\
10\end{array}$ & 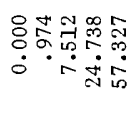 & 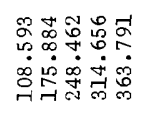 & 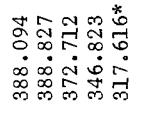 & 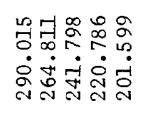 & 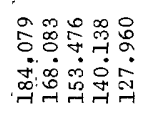 & 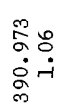 \\
\hline $\begin{array}{l}1 \\
\vdots \\
10 \\
1 \\
1 \\
1\end{array}$ & 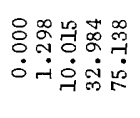 & 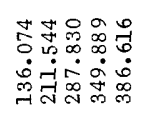 & 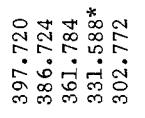 & 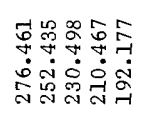 & 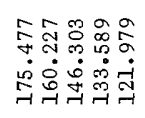 & 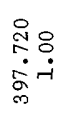 \\
\hline $\begin{array}{l}10 \\
10 \\
10 \\
10 \\
10\end{array}$ & 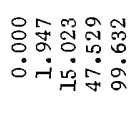 & 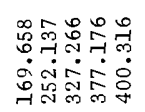 & 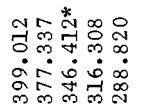 & 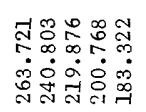 & 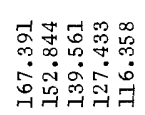 & 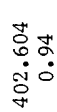 \\
\hline $\begin{array}{l}1-1 \\
\vdots \\
110 \\
10\end{array}$ & 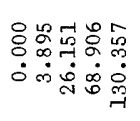 & 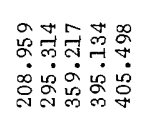 & 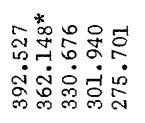 & 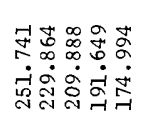 & 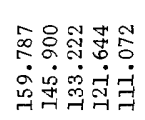 & 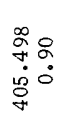 \\
\hline 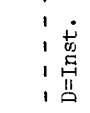 & 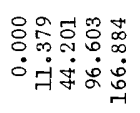 & 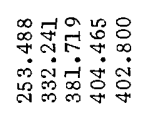 & 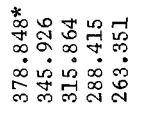 & 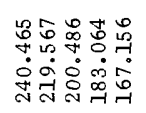 & 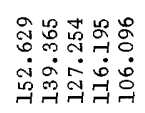 & 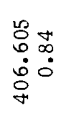 \\
\hline & 용유유윰유 & 웅요용요. & 우 & 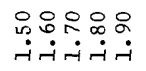 & ن & 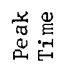 \\
\hline
\end{tabular}


10

1 i

1 E

1

1

,

10

1 芒

1

1 ह

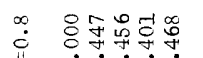

1

1 iI

15

10

告造这

80

1

1 II

14

1 II

1

'

, 年

1 ำ.

10

10

1

i

$1 \frac{1}{0}$

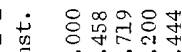

1 句

푀터

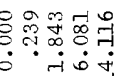

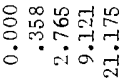

용ํำ

$\dot{m} \underset{\sim}{0} \stackrel{\infty}{\infty}$

- $\dot{\circ} \dot{7} \dot{0}$

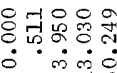

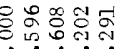

$\dot{0} \dot{\rightarrow} \dot{m}$

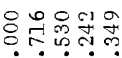

$\dot{0} \underset{\sim}{\stackrel{j}{y}}$

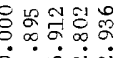

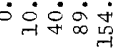

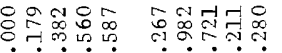

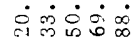

maे

तेंक्षित

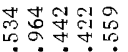

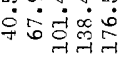

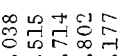

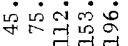

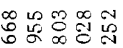

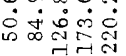

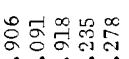

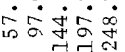

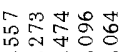

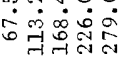

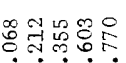

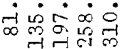

궁요융

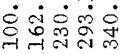

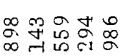

म्नें

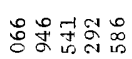

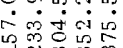

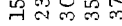

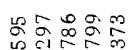

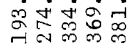

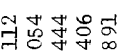

ம்

का

子小

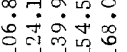

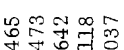

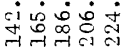

엉용ำ

में

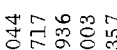

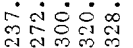

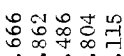

भिळ

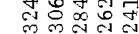

구워ํ영

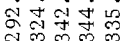

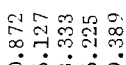

نं $\dot{0} \dot{0}$

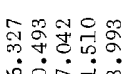

席总的我整

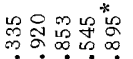

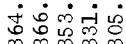

स:

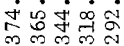

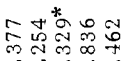

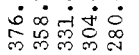

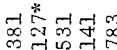

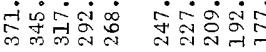

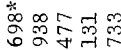

西

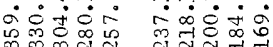

电号守售

คำ

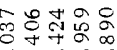

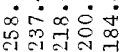

재워

से

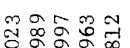

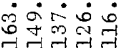

今ึ

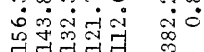

웅구욱융유

웅융요

옹유융유

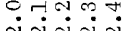

芦苛

垈

$\therefore$

$\underset{\substack{0 \\ \infty}}{-\infty}$

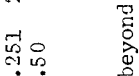

क्रु

获然兽

क्ष

$\stackrel{\infty}{\circ}$

$\dot{m} \dot{0}$

का

ॠి

क्षे

m० ठ

"이

品

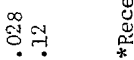

i

ت⿹

क्ष $-i$

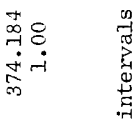

娄요

$\underset{\infty}{\infty} \stackrel{\infty}{\infty} \quad$ है

के

更

요

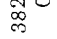

荬. 


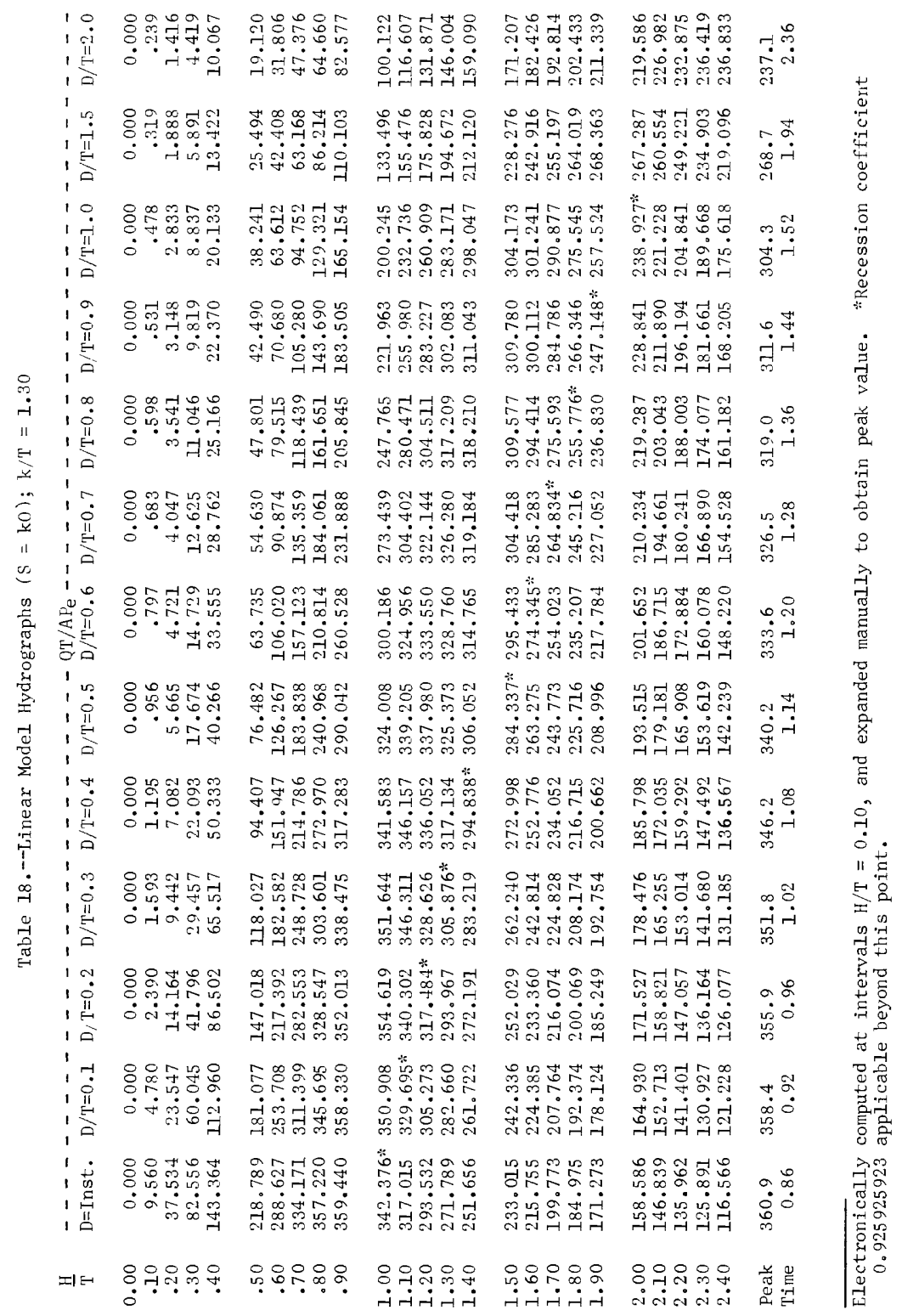




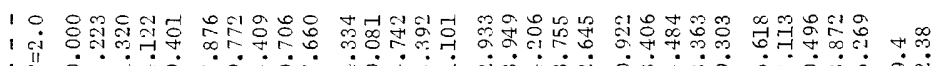
, لُ

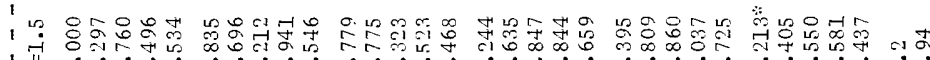

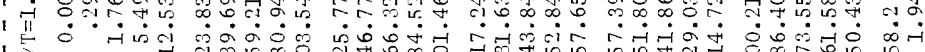
,

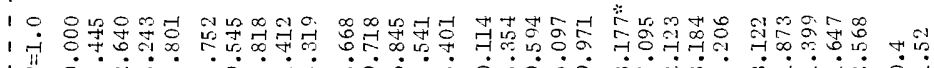
|

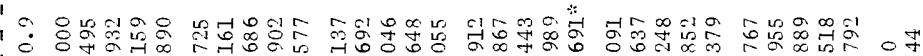
II

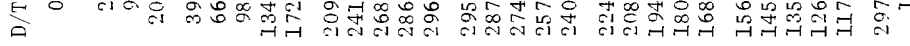

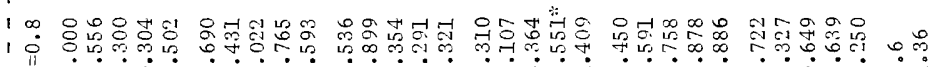

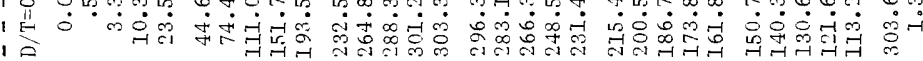

I:

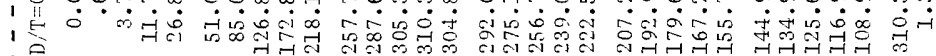

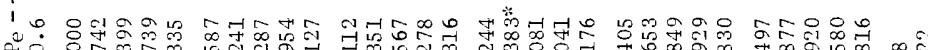

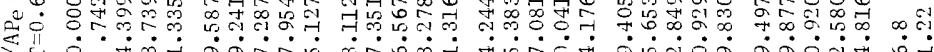

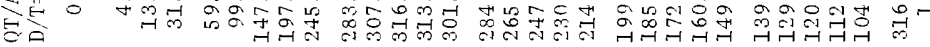

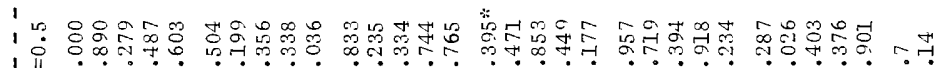

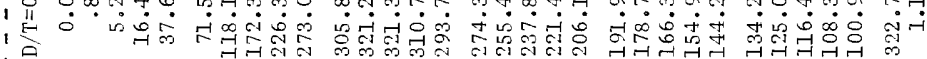

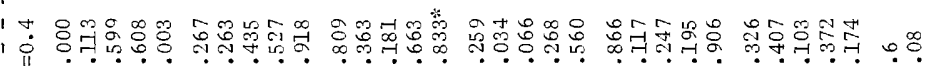

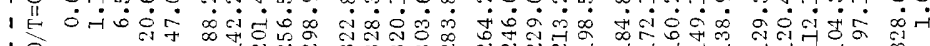
1 .

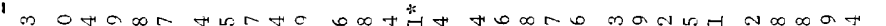
光: |

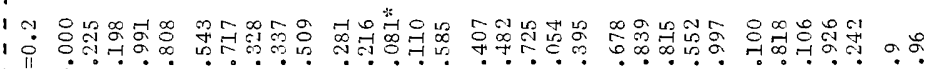

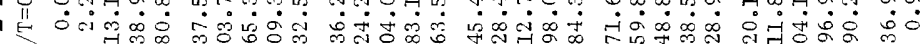
18

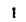

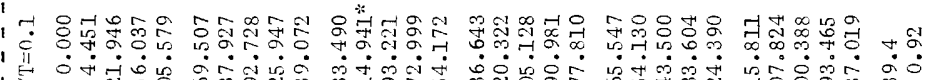

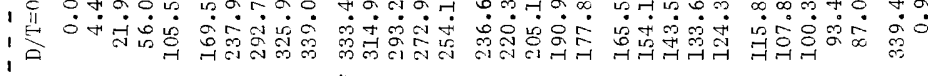

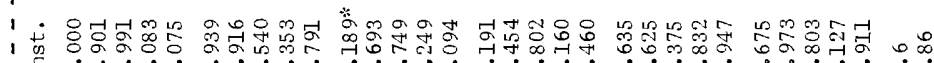

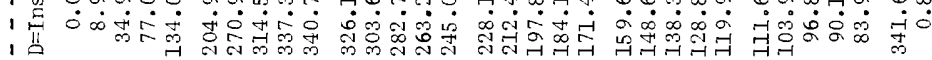

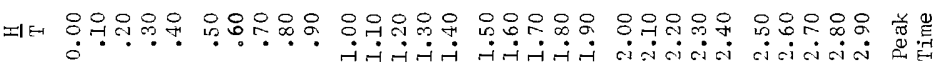




\begin{tabular}{|c|c|c|c|c|c|c|}
\hline $\begin{array}{ll}1 & 0 \\
1 & i \\
1 & 11 \\
1 & 1\end{array}$ & 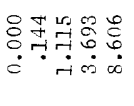 & 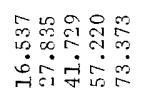 & 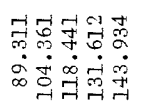 & 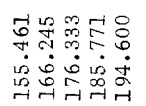 & 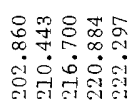 & 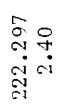 \\
\hline 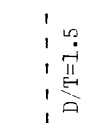 & 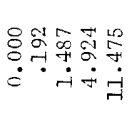 & 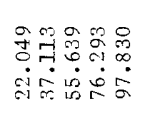 & 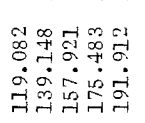 & 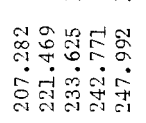 & 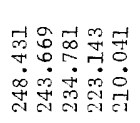 & 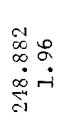 \\
\hline $\begin{array}{l}10 \\
10 \\
11 \\
10\end{array}$ & 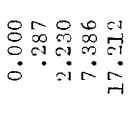 & 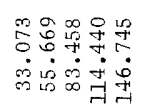 & 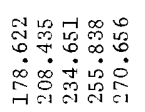 & 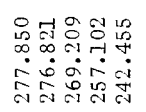 & 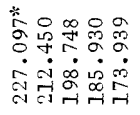 & 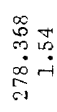 \\
\hline : & 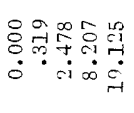 & 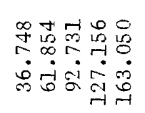 & 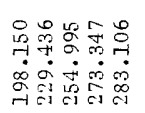 & 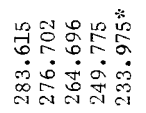 & 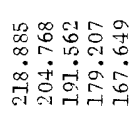 & 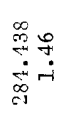 \\
\hline $\begin{array}{l}1 \infty \\
1 \stackrel{0}{0} \\
1 \\
1 \\
1\end{array}$ & 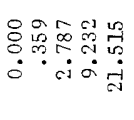 & 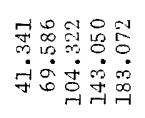 & 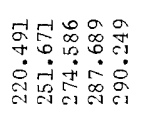 & 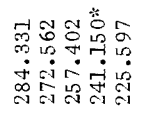 & 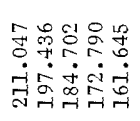 & 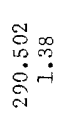 \\
\hline i⿱ & 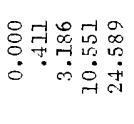 & 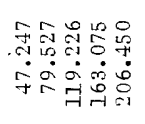 & 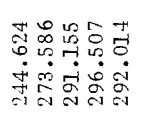 & 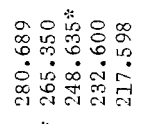 & 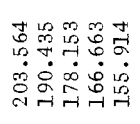 & 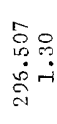 \\
\hline 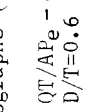 & 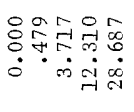 & 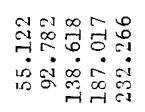 & 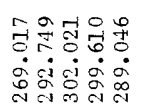 & 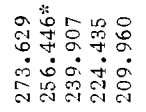 & $\begin{array}{l}\infty \\
\infty \\
7\end{array}$ & 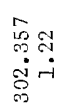 \\
\hline 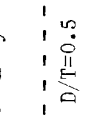 & 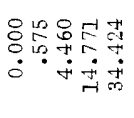 & 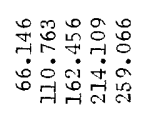 & 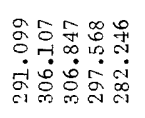 & 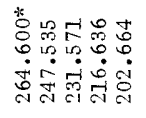 & 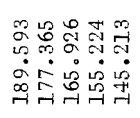 & 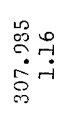 \\
\hline $\begin{array}{l}1 \stackrel{+}{0} \\
1 \\
1 \\
1 \\
1\end{array}$ & 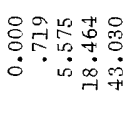 & 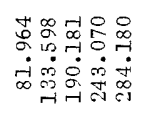 & 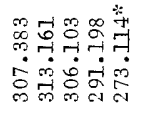 & 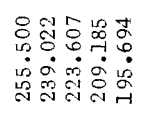 & 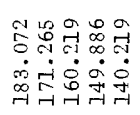 & 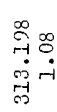 \\
\hline $\begin{array}{l}1 \\
1 \\
1 \\
1 \\
1 \\
1\end{array}$ & 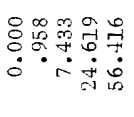 & 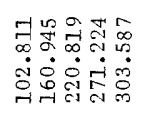 & 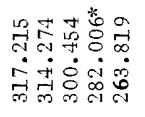 & 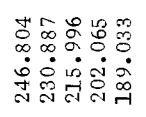 & 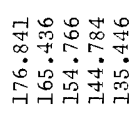 & 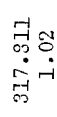 \\
\hline $\begin{array}{ll}1 & 0 \\
1 & 0 \\
1 & 11 \\
10\end{array}$ & 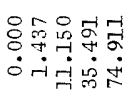 & 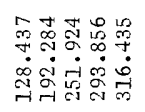 & 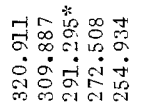 & 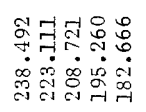 & 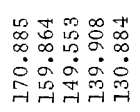 & 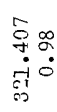 \\
\hline :- & 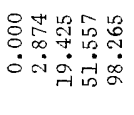 & 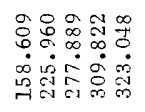 & 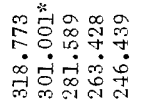 & 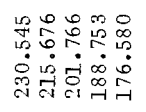 & 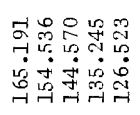 & 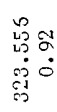 \\
\hline is & 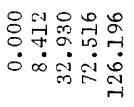 & 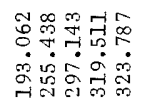 & 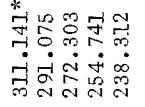 & 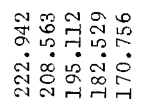 & 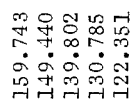 & 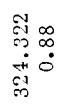 \\
\hline 피타 & 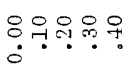 & 윰요요요. & 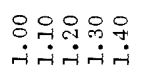 & 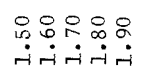 & 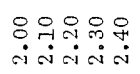 & 总唇 \\
\hline
\end{tabular}




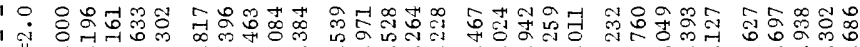

I II

10

1 ᄂ

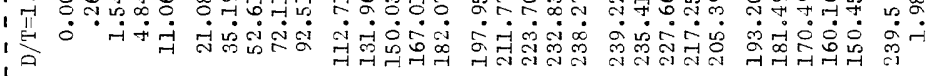

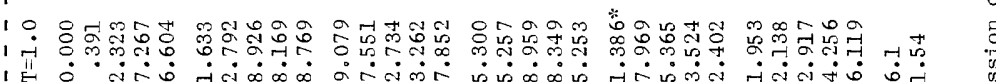

I

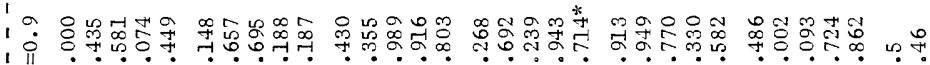

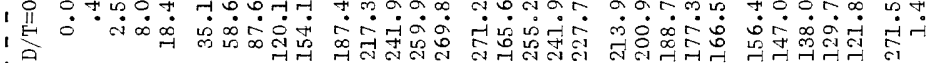

1

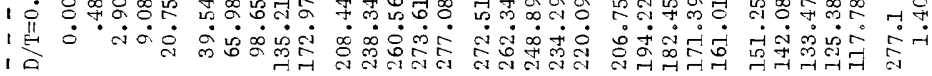

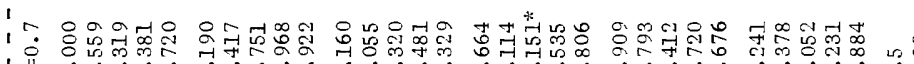

I

।

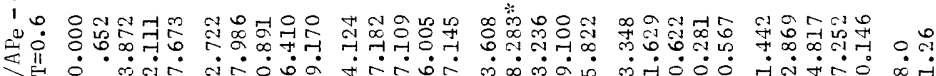

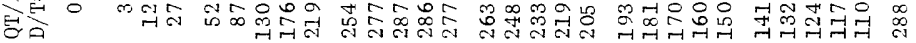

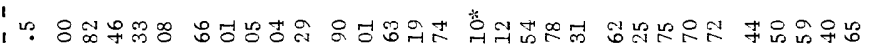

II \&0ํำ

| 合

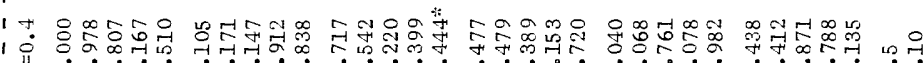

1 $\begin{aligned} & 110000 \\ & 1\end{aligned}$

la क्न म

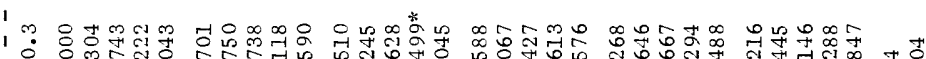

। II

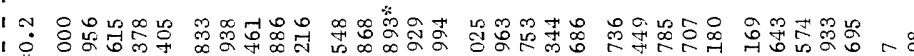

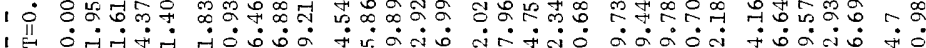

।

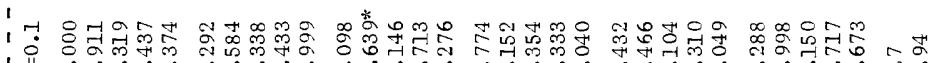

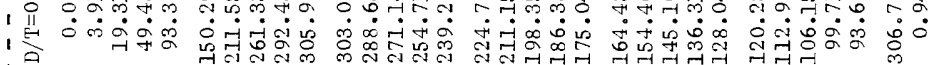

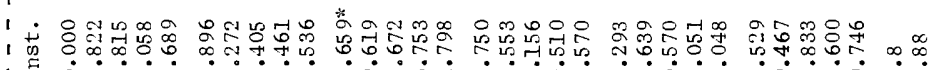

। 1 il

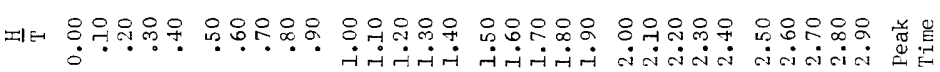




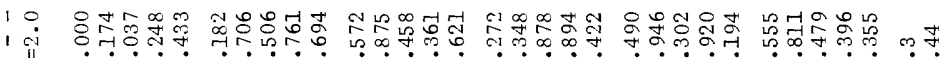

! II

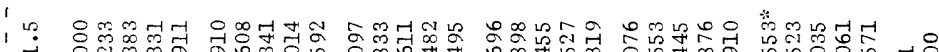

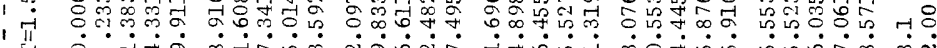

:

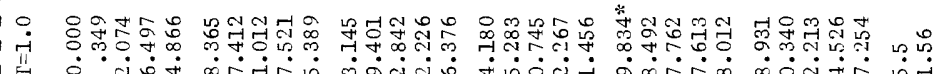

l

10

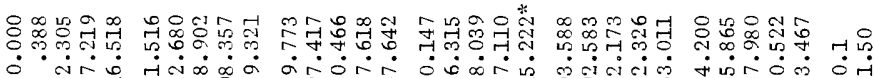

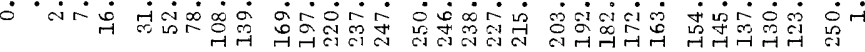

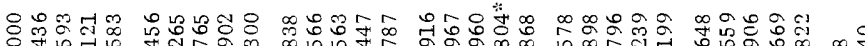

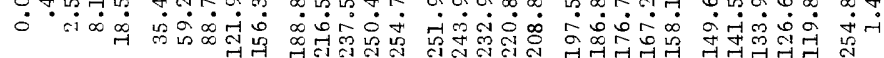

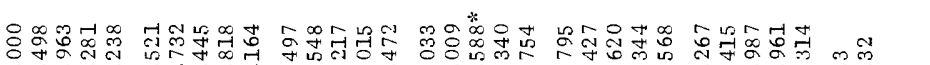

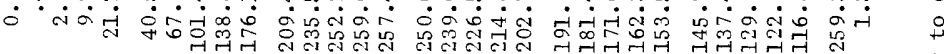

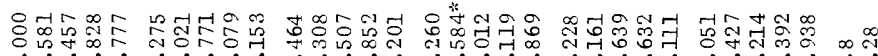

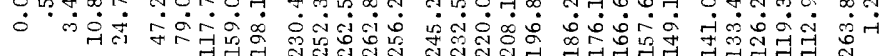

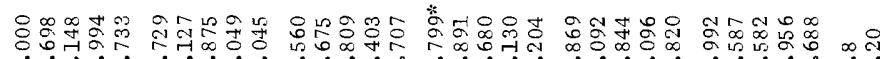

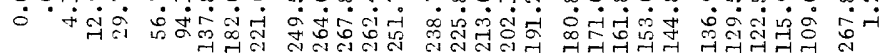

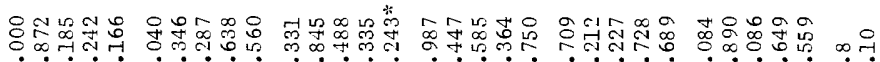
○ ம்

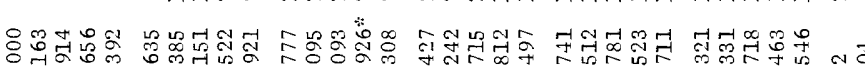

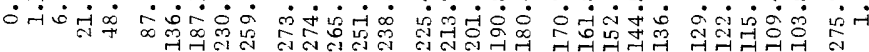

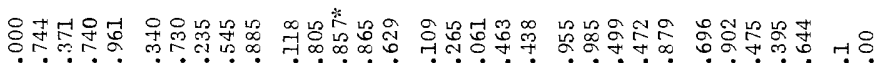
ல்

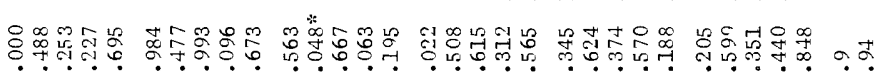

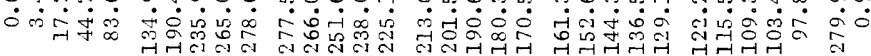

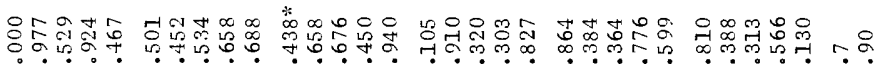

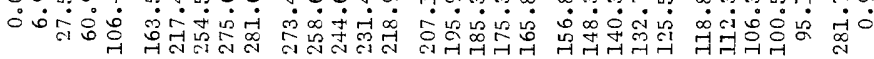




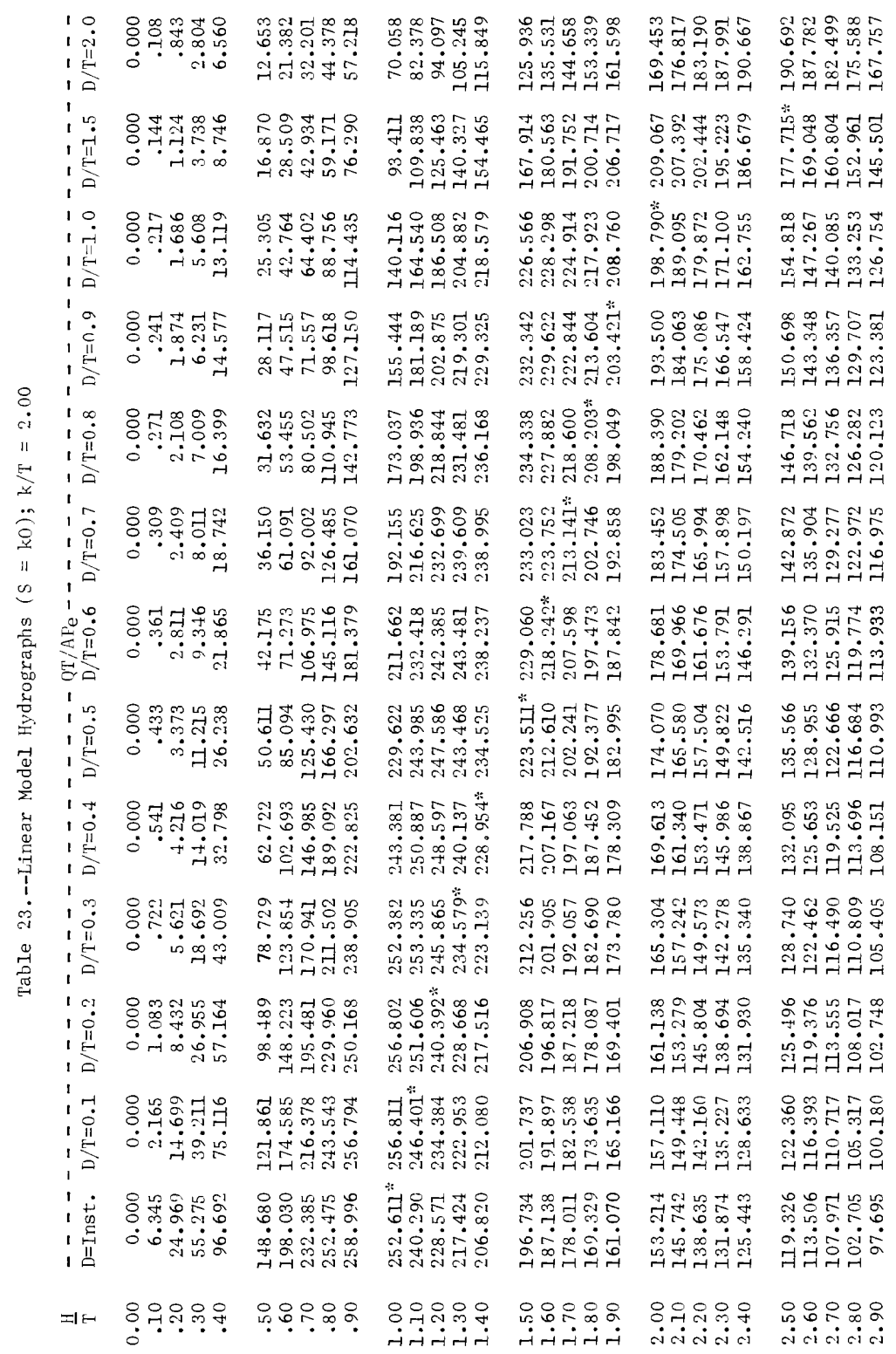




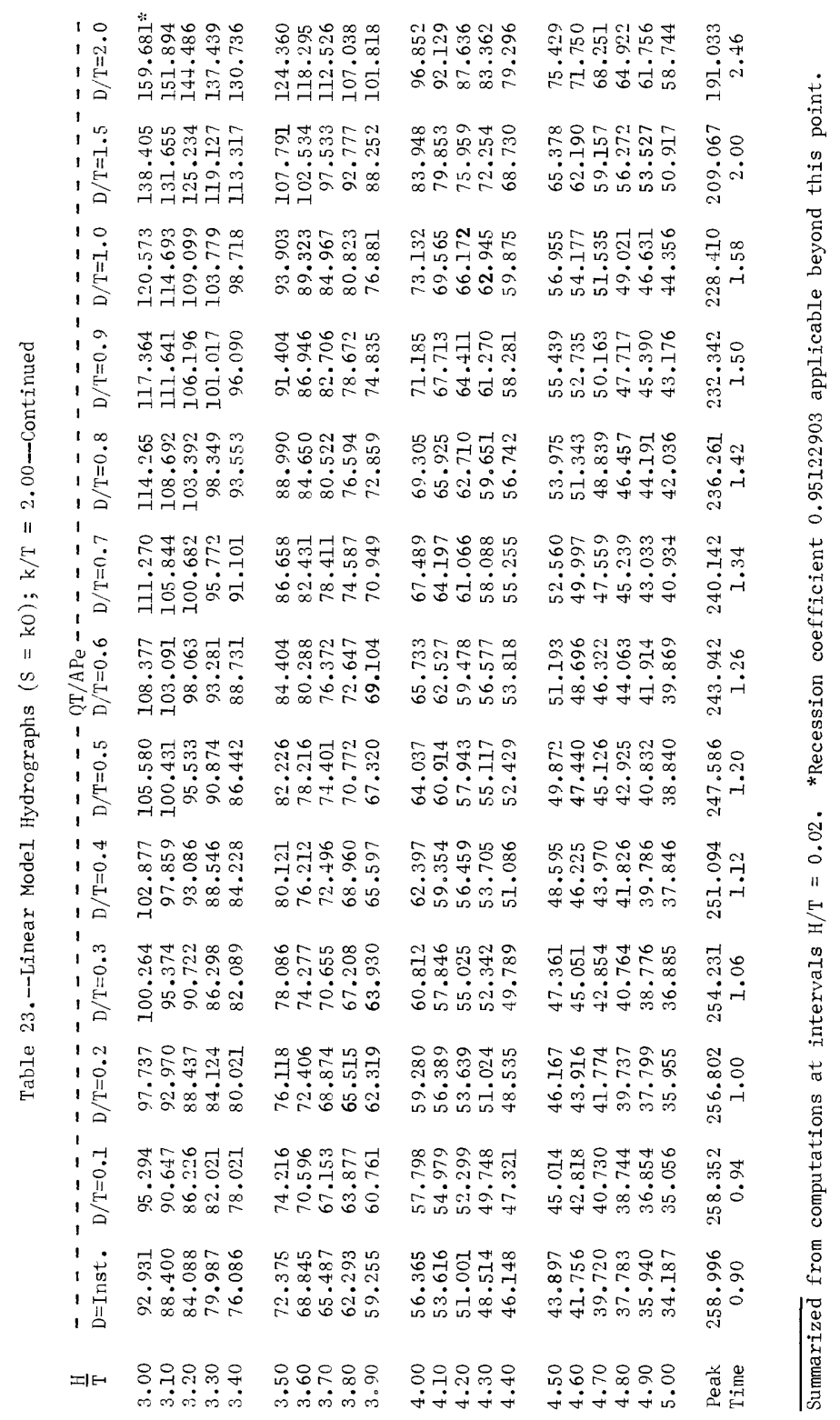




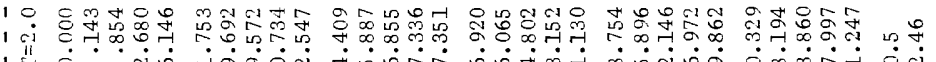
I

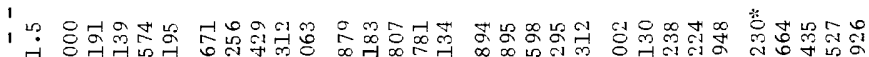

।

, 至

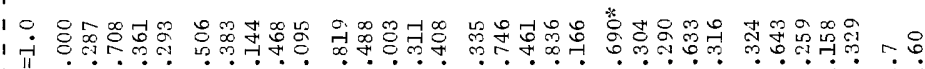

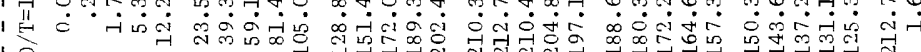

$1 \mathrm{a}$

la o우

1 ।

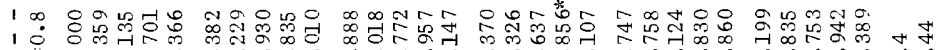

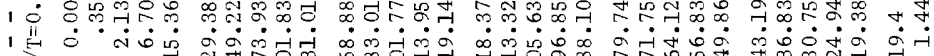
I 15 N

1

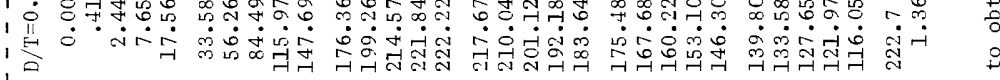

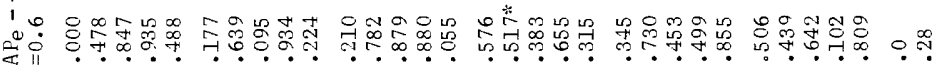

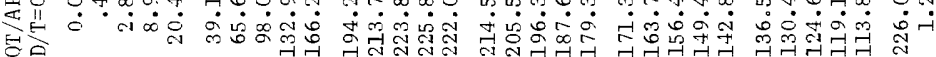

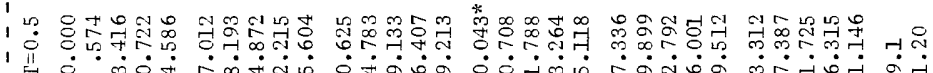

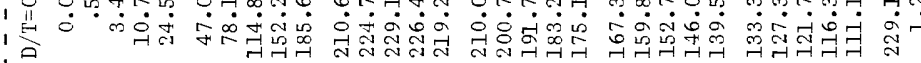

।

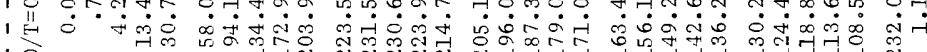

$\infty 80+8$

on

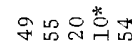

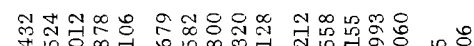

1

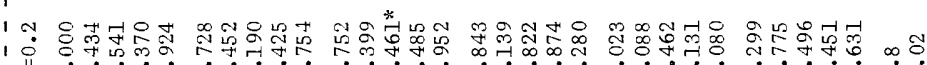
l 1 l

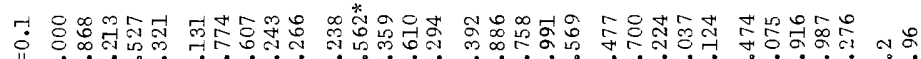

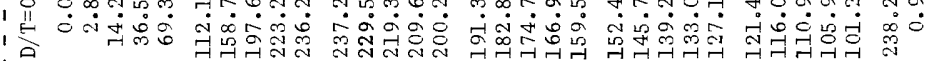
' , 尚

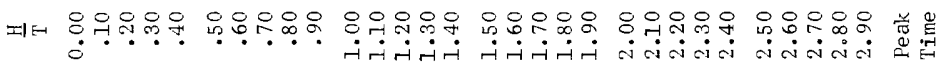




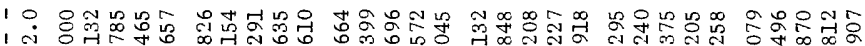

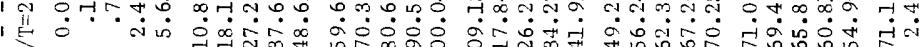

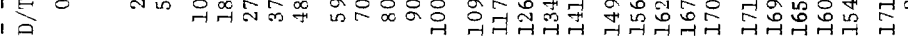

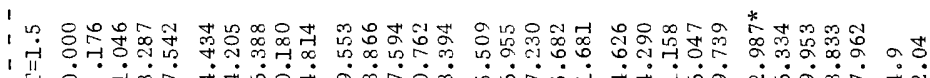

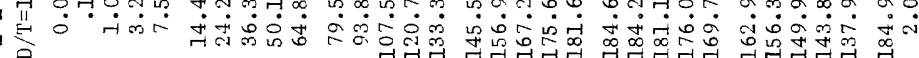

1

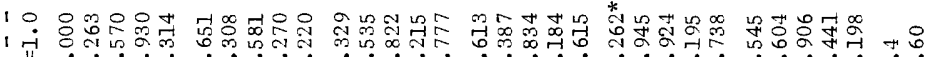

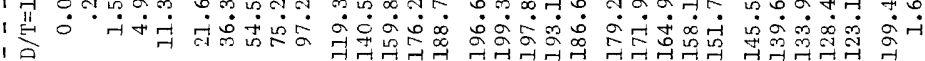

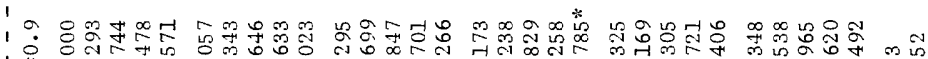

1I

1.

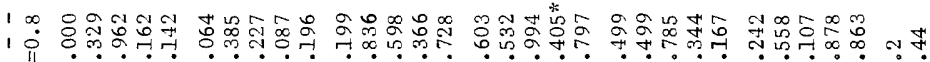

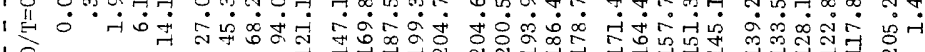

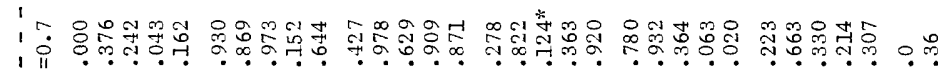

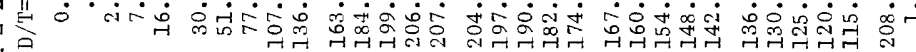

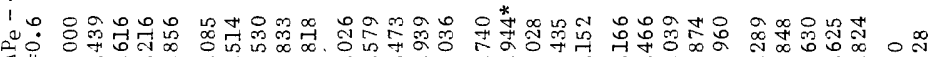
綮向

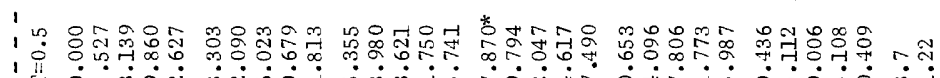

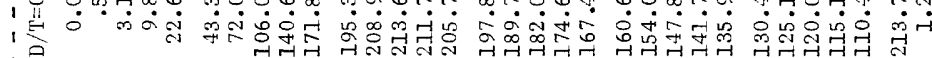

1

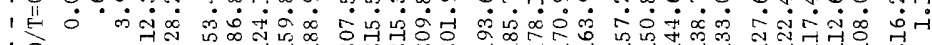

10

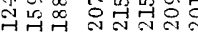

!

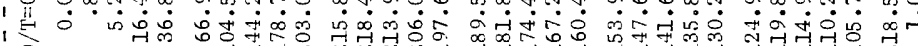

1

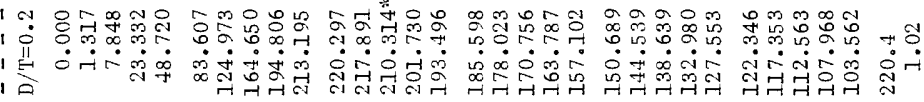

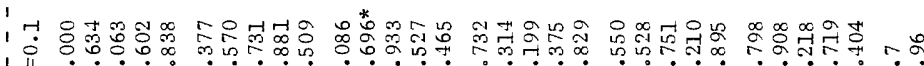

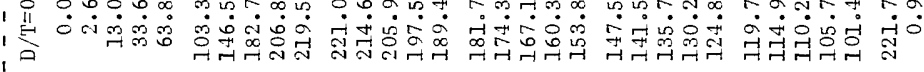

1

1 䓃

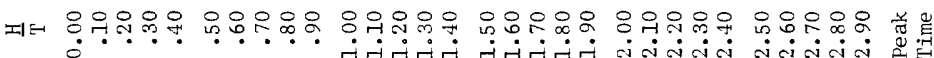




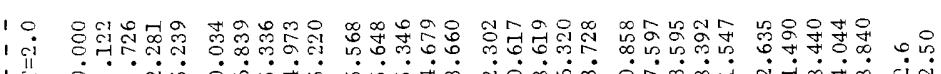

l少

1 is

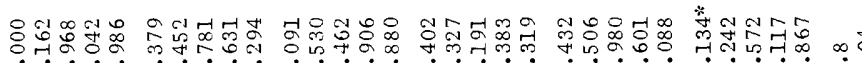

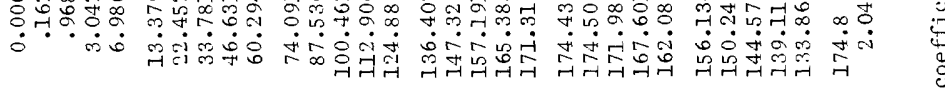

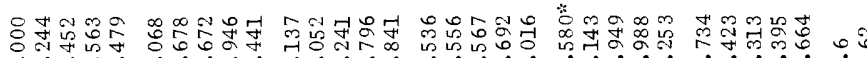
○

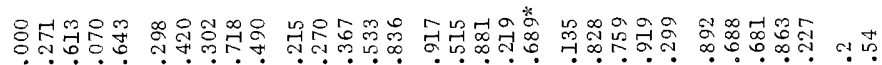

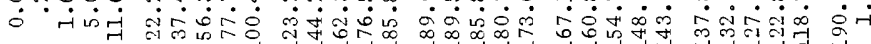

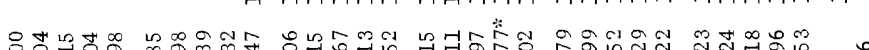

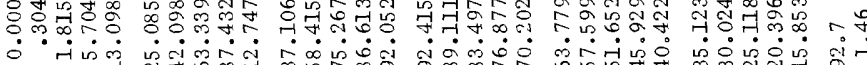

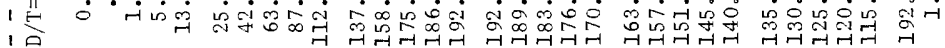

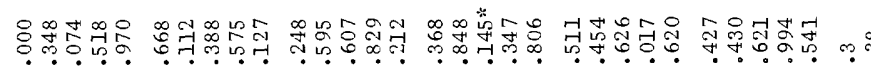

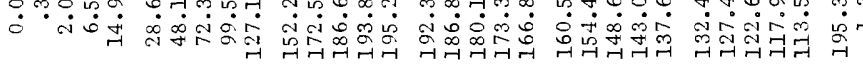

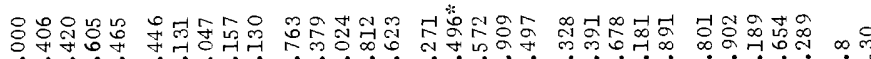
○ N穴

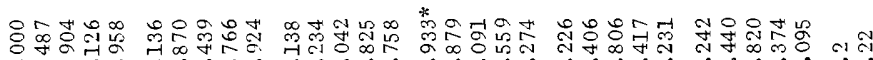

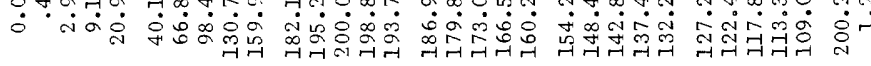

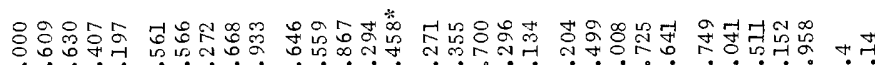

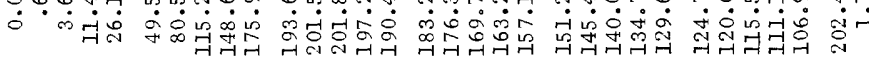

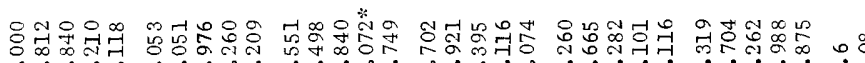

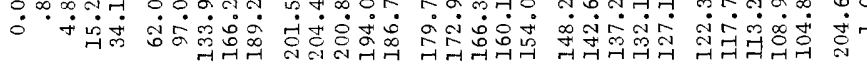
务

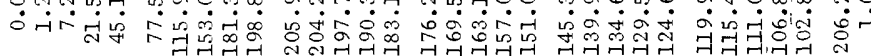

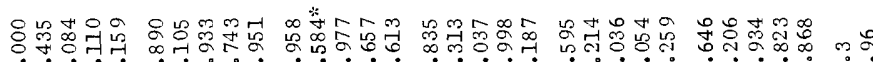

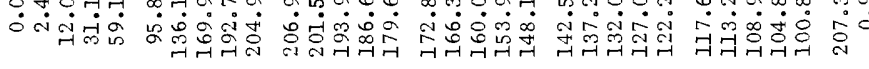

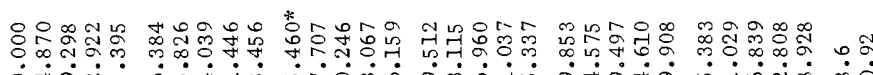

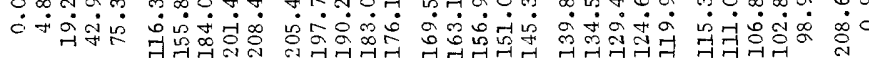

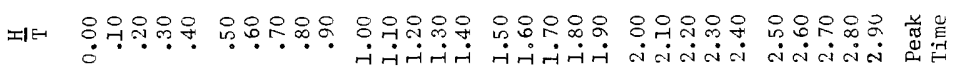




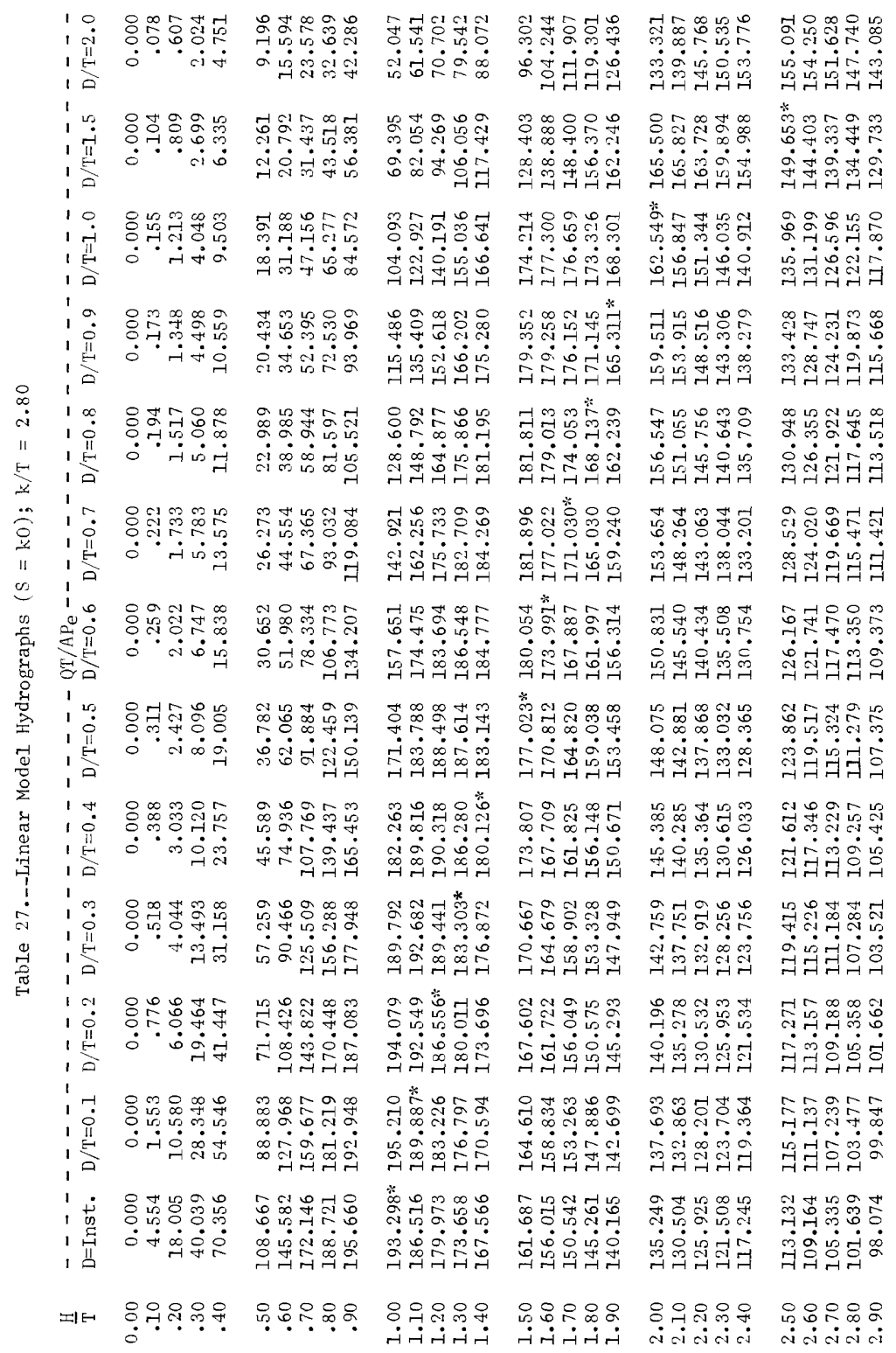




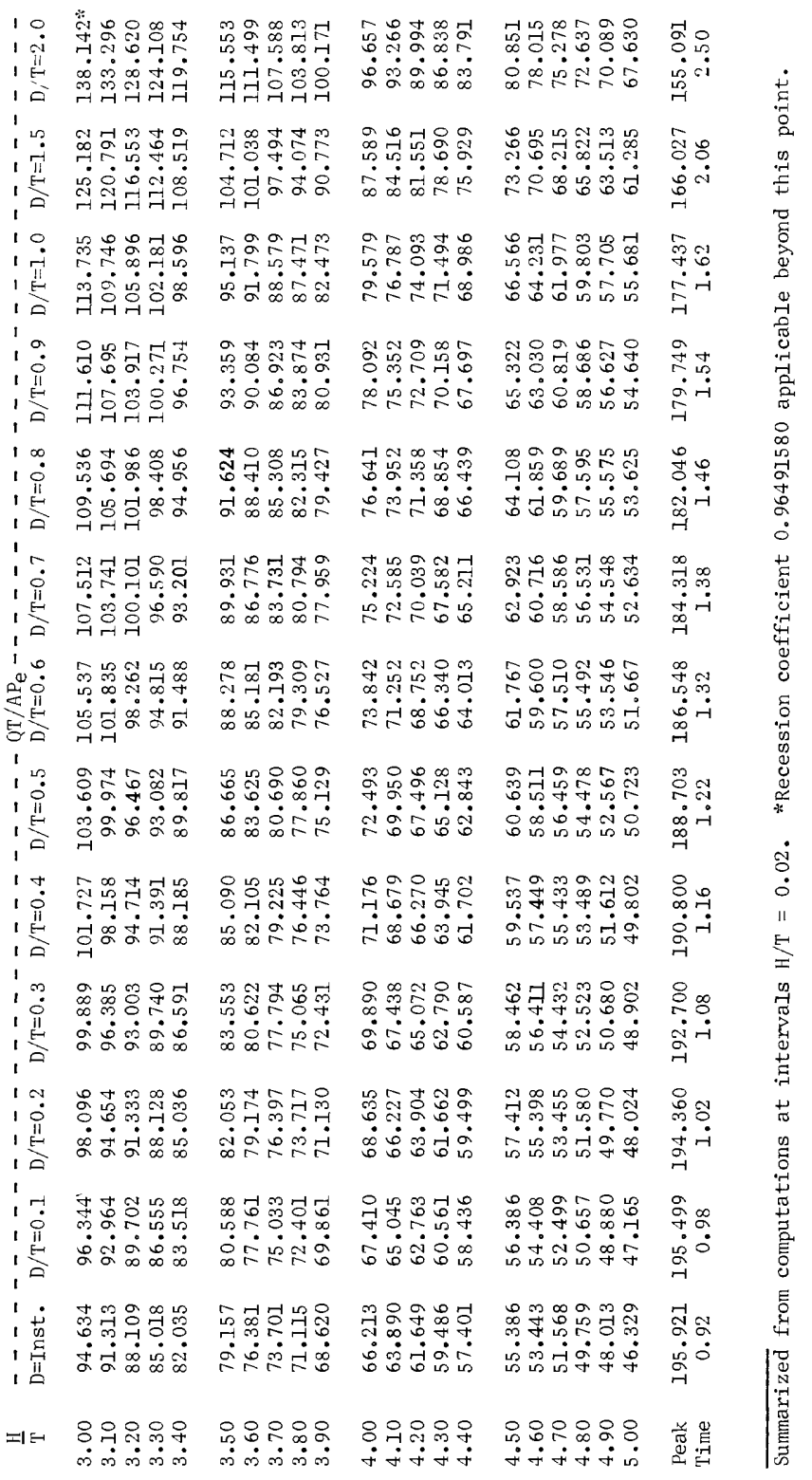




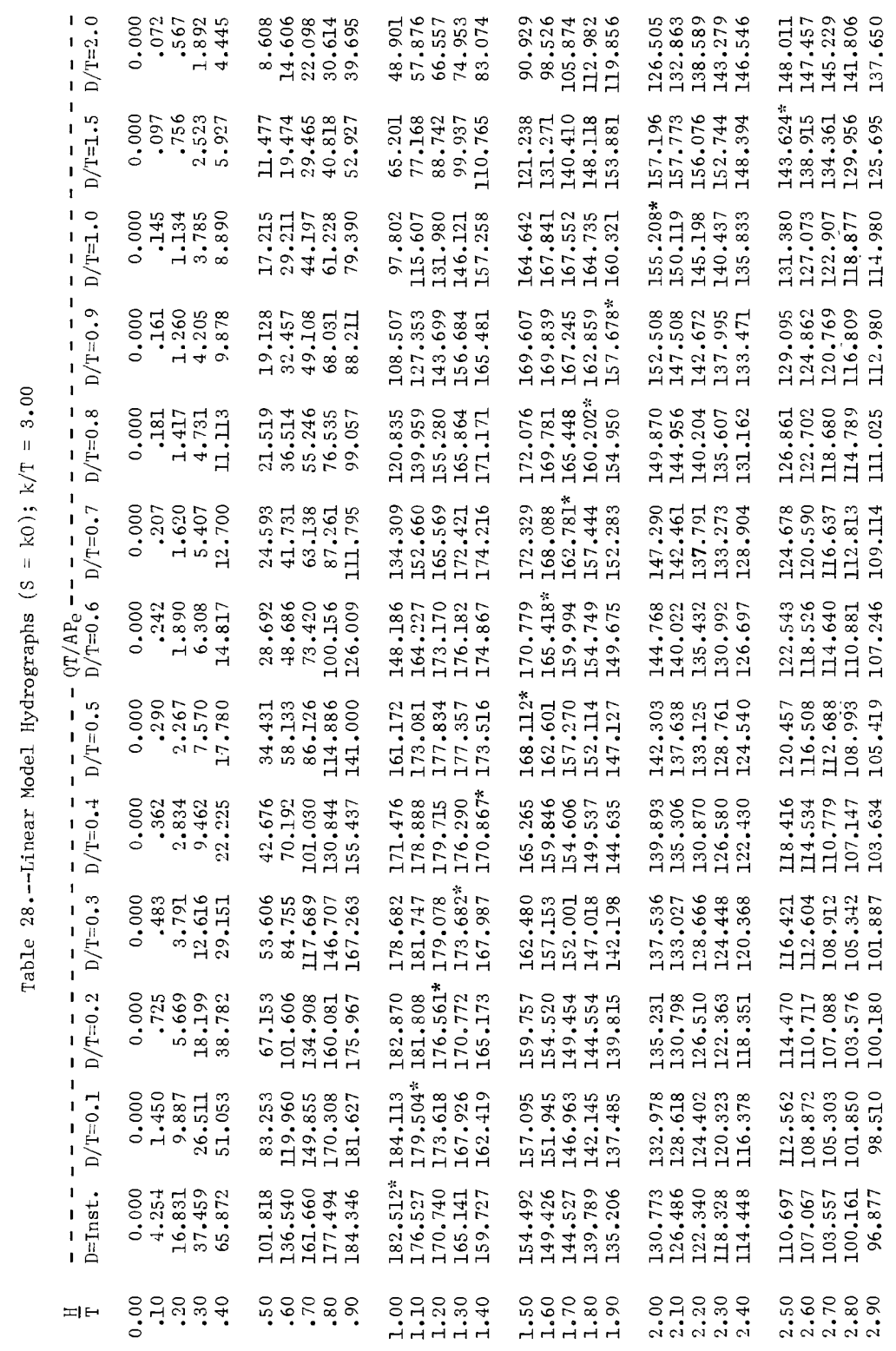




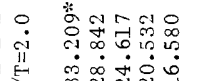

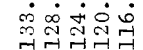

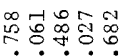

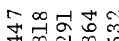

2010

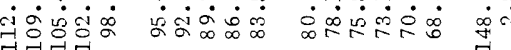

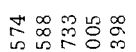

웅슈요

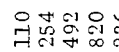

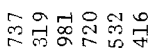
.

걱형 (1) में $\dot{\infty} \dot{\infty} \dot{\infty}$

रंसंळ

$\stackrel{\infty}{\infty}$

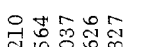

تं宀 $\dot{0} \dot{0}$

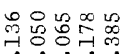

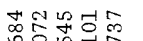

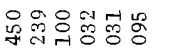

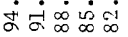

$\sin \dot{x} \sin$

ธิ்่

过

is

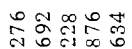

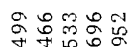

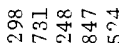

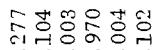

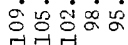

نं $\dot{0} \dot{0}$

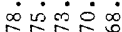

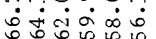

蓄象

궁

น 궁

:

a $\infty \infty \infty$

m-1 $\rightarrow$ -

가요요

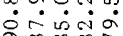

$\dot{\circ} \dot{0}$

जिव:

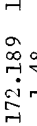

둥응육영

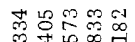

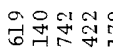

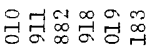

$\dot{\infty} \dot{\infty} \dot{\infty} \infty$

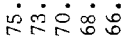

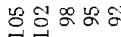

은

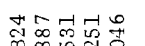

पुं

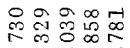

க்형여

다잉

मेंवे

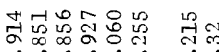

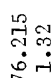

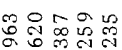

ப் 영혀

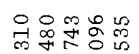

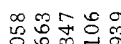

तi

$\underset{7}{\rightarrow}$

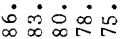

rin

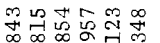

$\stackrel{-90}{\circ}$

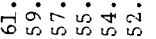

வำ

웜ㄷㅈㅔ

舟웅용

겅요

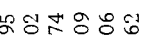

क्ष

ठ்

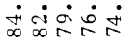

सं०ेंघं

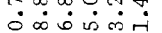

$\dot{\infty}$

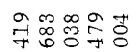

궁요성

구의

突음

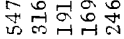

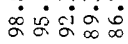

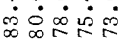

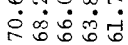

करें

걱-

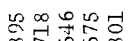

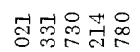

둥욤ㄷㅇ

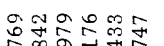

$\infty$

فळ욯

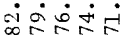

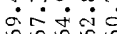

क人ष

लं

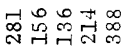

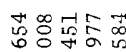

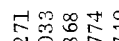

용ํㅛㅇㅇㅇ음

요

शून $\sigma_{\infty}^{\infty} \infty$

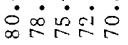

क்

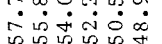

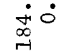

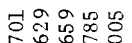

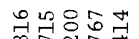

舟产高品

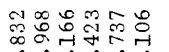

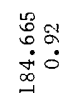

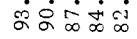

वंक्षंत्र

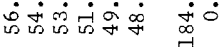

$=1 \mathrm{H}$

울유듀.

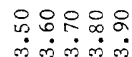

욱윰유

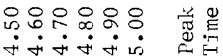




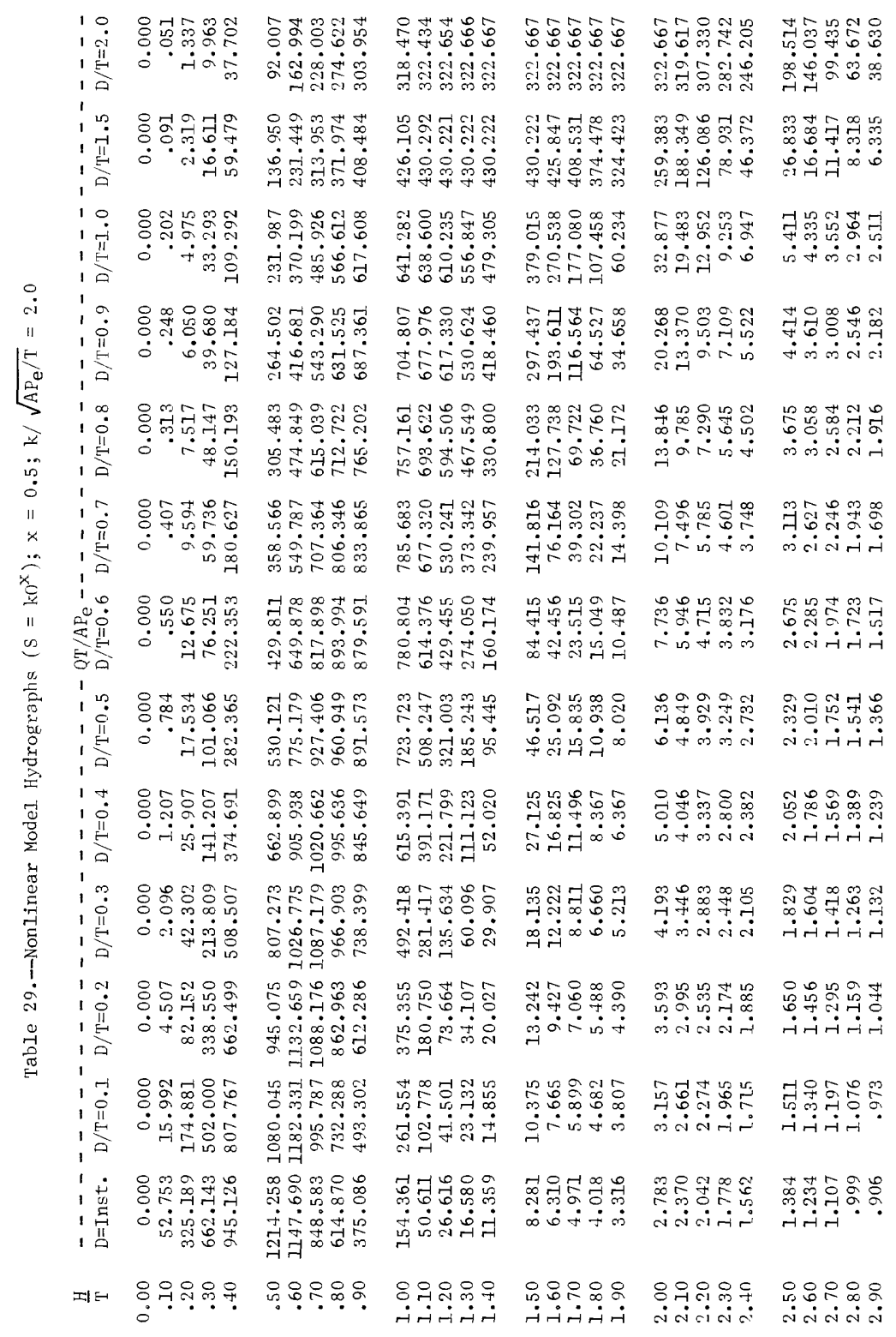




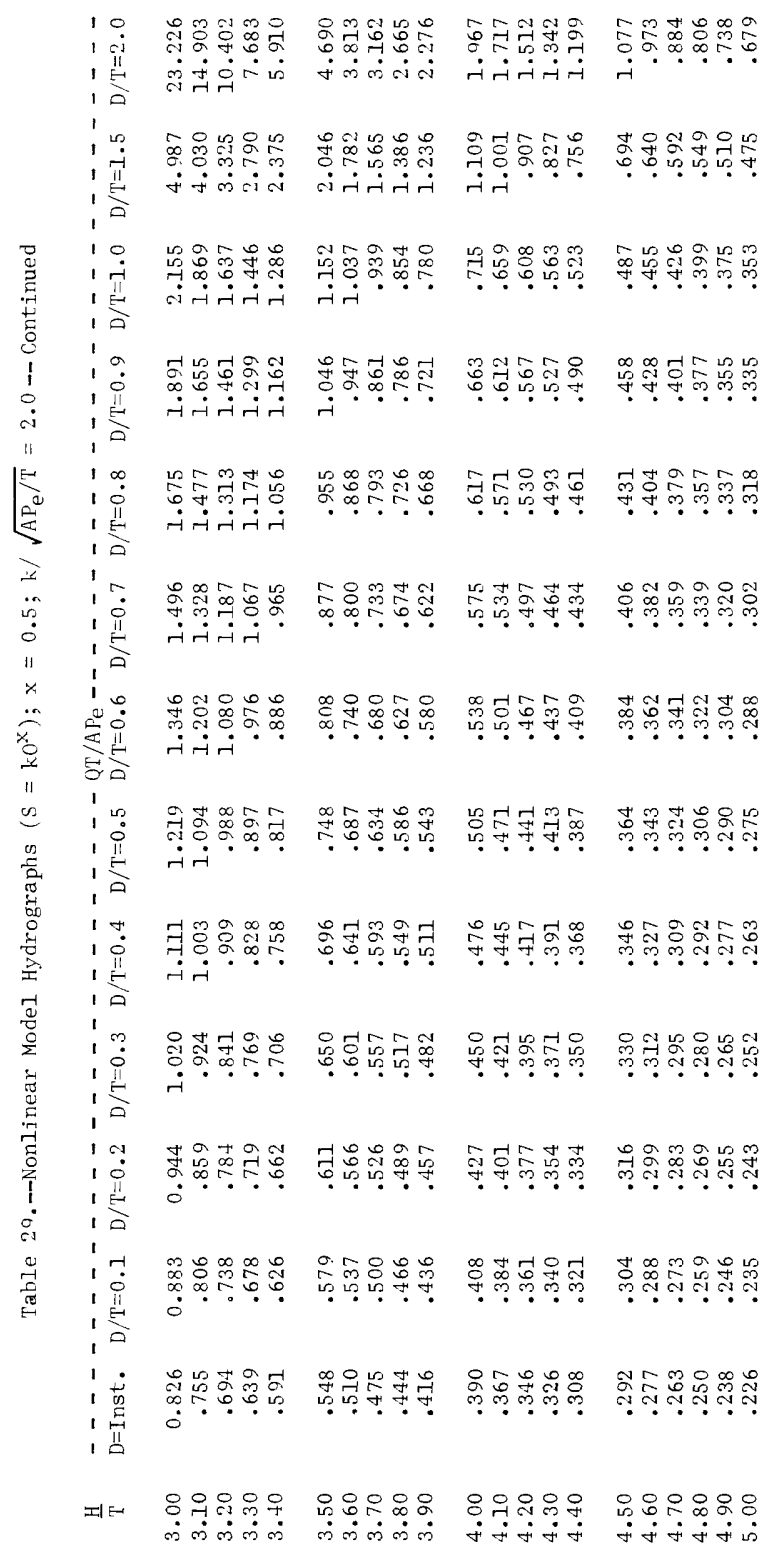




\begin{tabular}{|c|c|c|c|c|c|c|}
\hline $\begin{array}{l}10 \\
100 \\
1 \\
10 \\
10\end{array}$ & $\begin{array}{l}8 \\
\vdots \\
\vdots\end{array}$ & 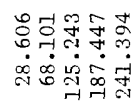 & 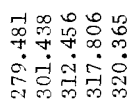 & 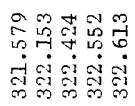 & 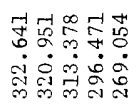 & 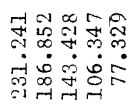 \\
\hline : & 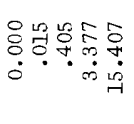 & 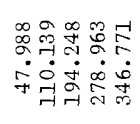 & 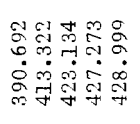 & 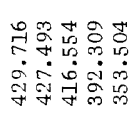 & 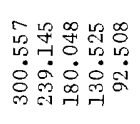 & 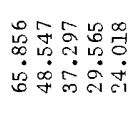 \\
\hline $\begin{array}{l}10 \\
1 \stackrel{4}{11} \\
1 \\
10\end{array}$ & 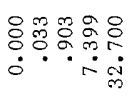 & 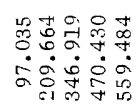 & 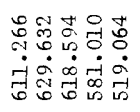 & 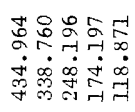 & 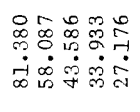 & 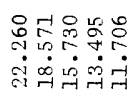 \\
\hline $\begin{array}{l}1 \\
10 \\
10 \\
1 \\
1\end{array}$ & 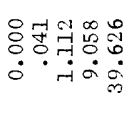 & 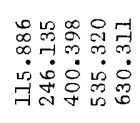 & 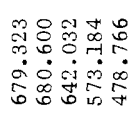 & 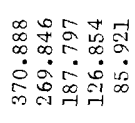 & 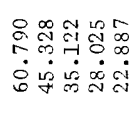 & 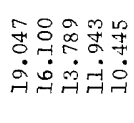 \\
\hline $\begin{array}{ll}1 & \infty \\
1 & 0 \\
11 \\
1 \\
1\end{array}$ & & 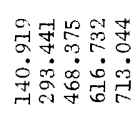 & 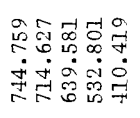 & 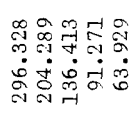 & 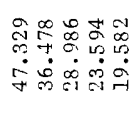 & 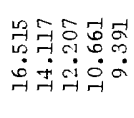 \\
\hline ì & 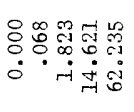 & 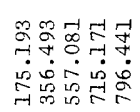 & 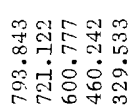 & 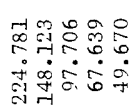 & 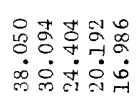 & $\begin{array}{l}\infty \\
\infty \\
\infty \\
+\end{array}$ \\
\hline 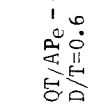 & & 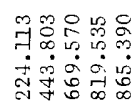 & 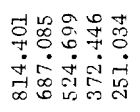 & 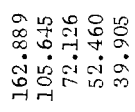 & 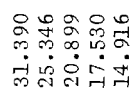 & 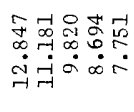 \\
\hline $\begin{array}{ll}1 & 0 \\
1 & 0 \\
11 \\
11 \\
1\end{array}$ & $\begin{array}{l}8 \\
8 \\
0 \\
0 \\
0 \\
0\end{array}$ & 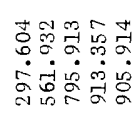 & 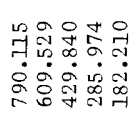 & 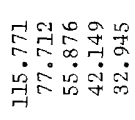 & 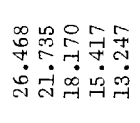 & 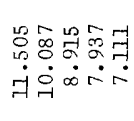 \\
\hline 吾 & & 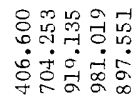 & 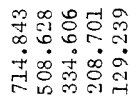 & 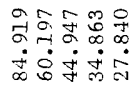 & 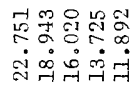 & 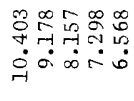 \\
\hline : & 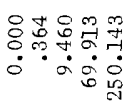 & 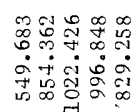 & 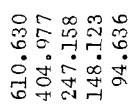 & 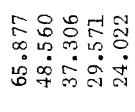 & 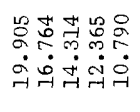 & 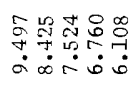 \\
\hline $\begin{array}{l}10 \\
10 \\
1 \\
11 \\
10 \\
10\end{array}$ & 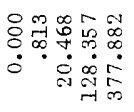 & 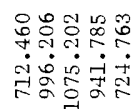 & 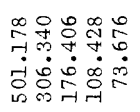 & 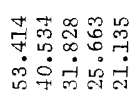 & 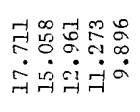 & 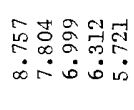 \\
\hline $\begin{array}{l}1 \\
1 \\
1 \\
1 \\
1\end{array}$ & 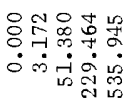 & 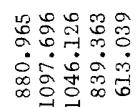 & 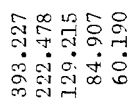 & 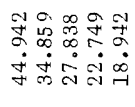 & 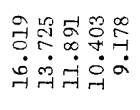 & 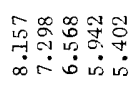 \\
\hline $\begin{array}{l}1 \\
1 \\
100 \\
1 \\
100\end{array}$ & 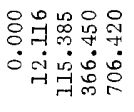 & 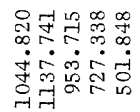 & 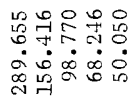 & 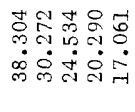 & 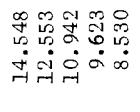 & 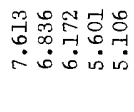 \\
\hline & 용윔유유유. & 우윰ำ & & & & \\
\hline
\end{tabular}




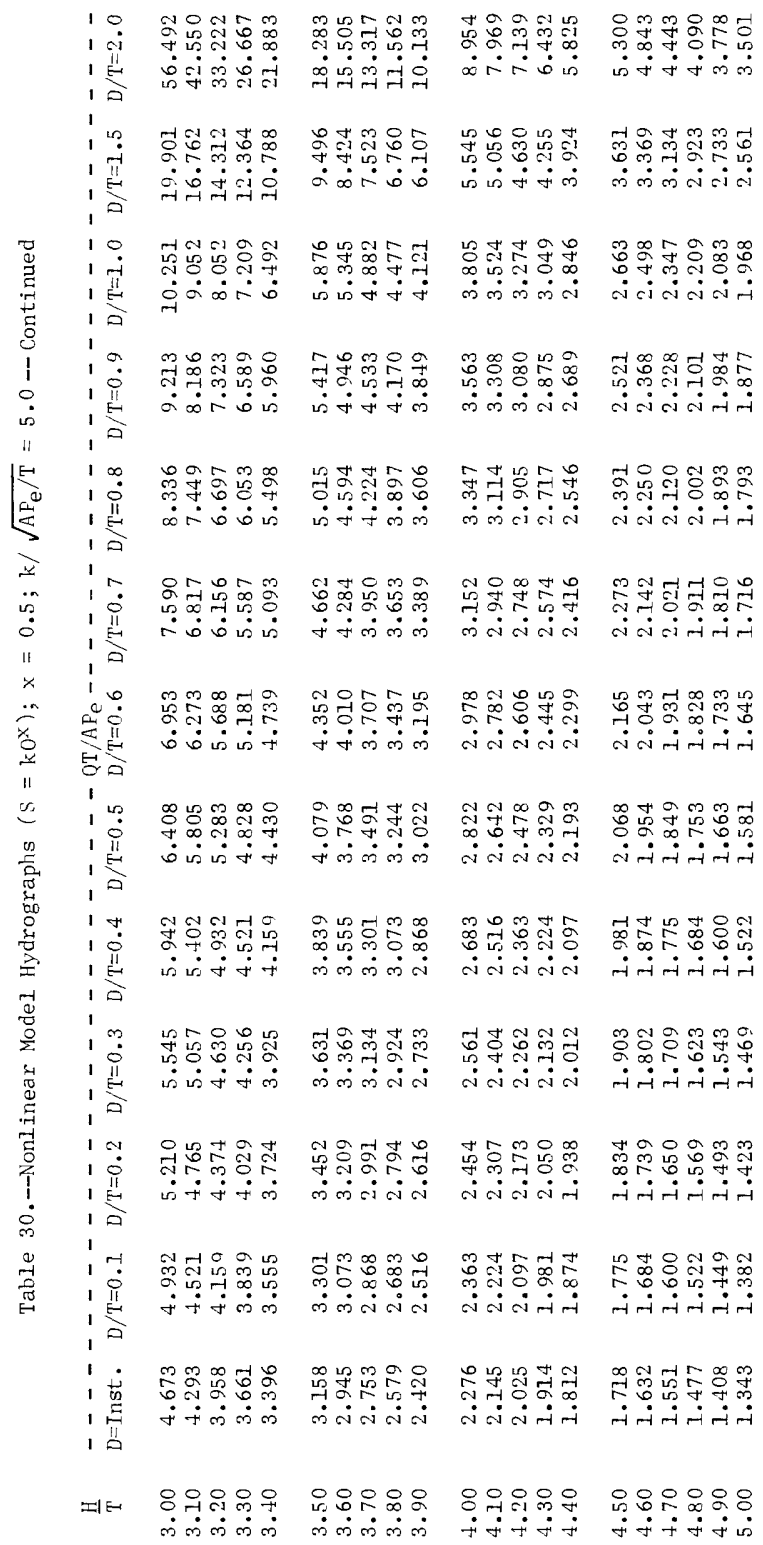




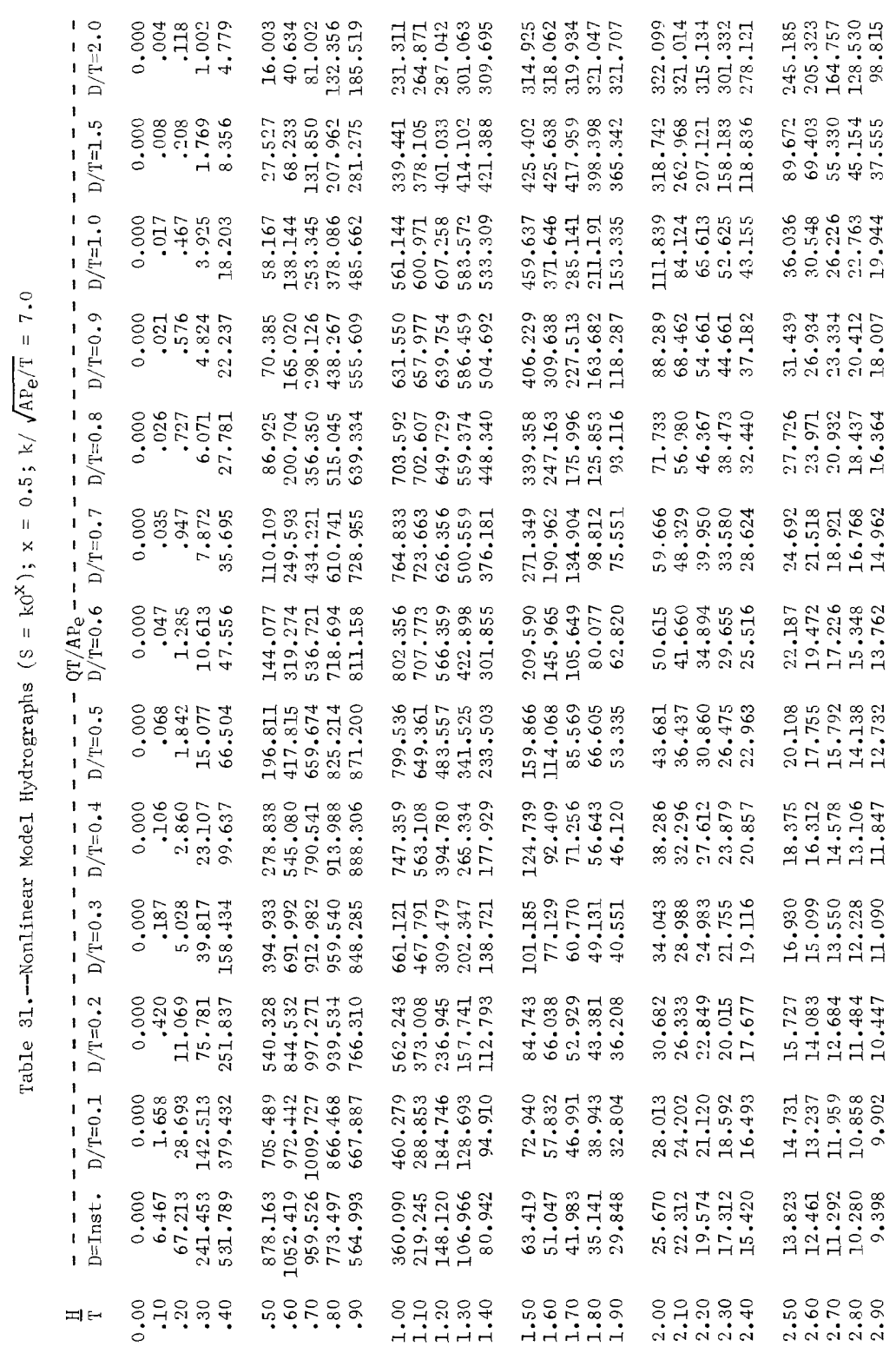




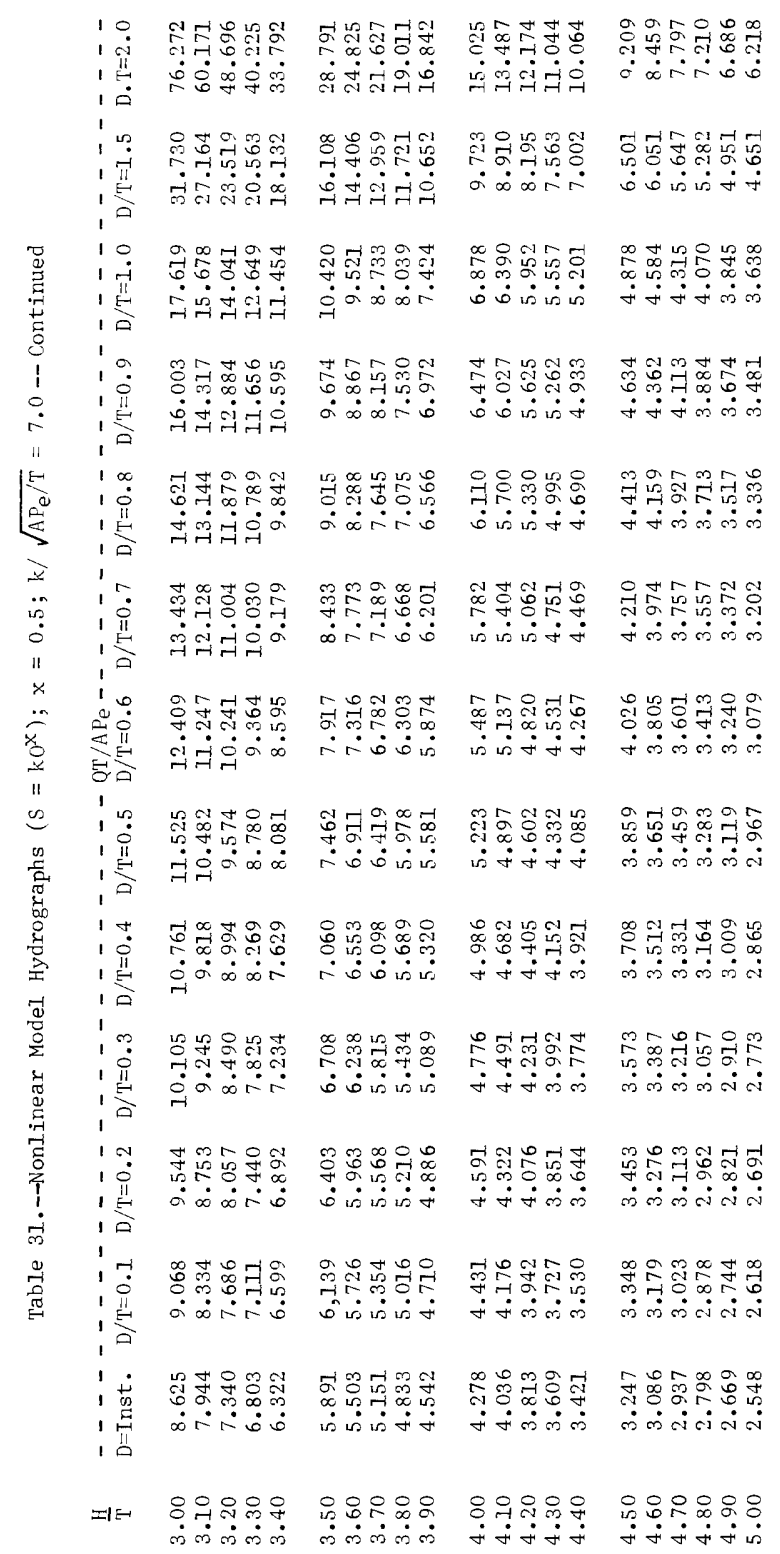




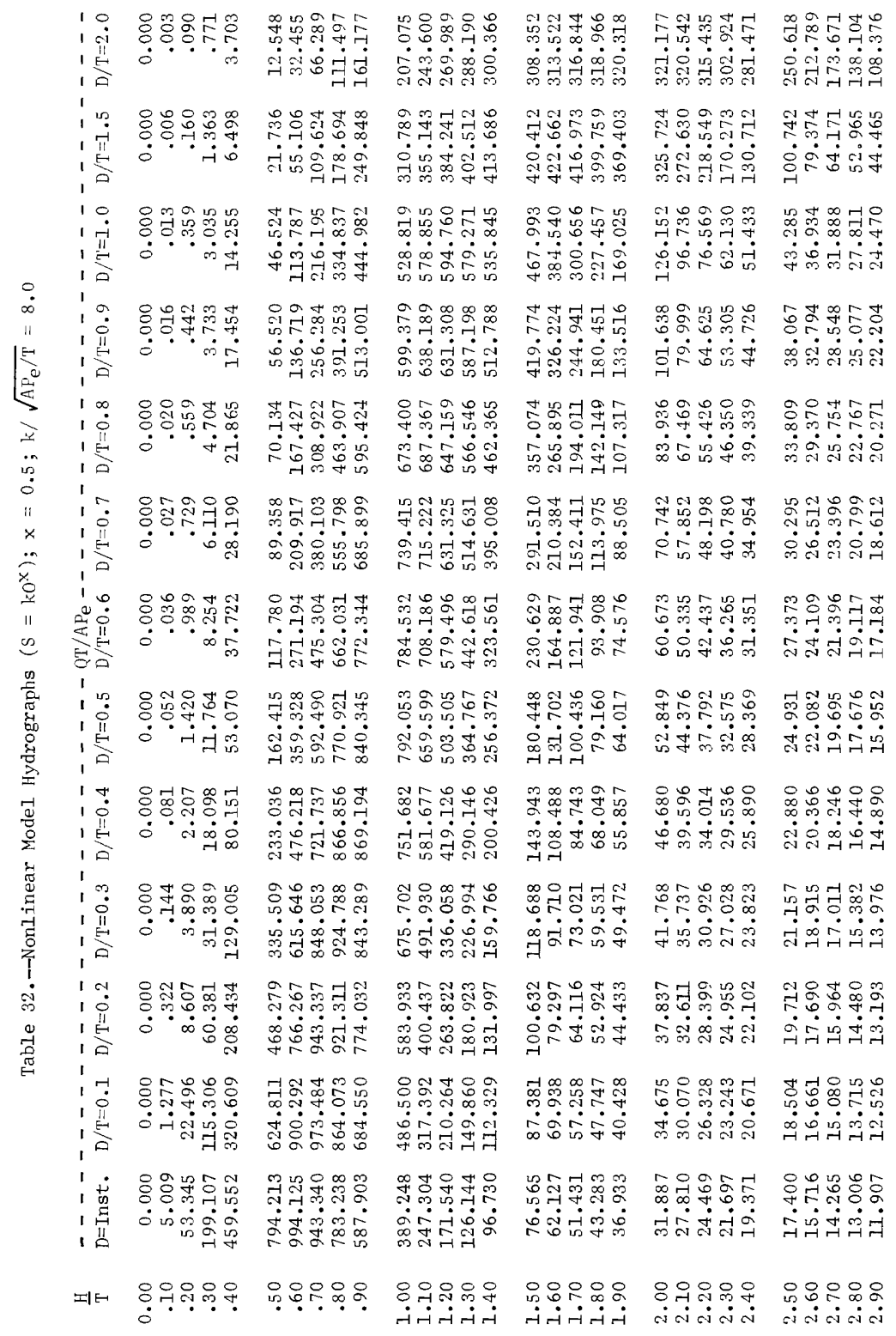




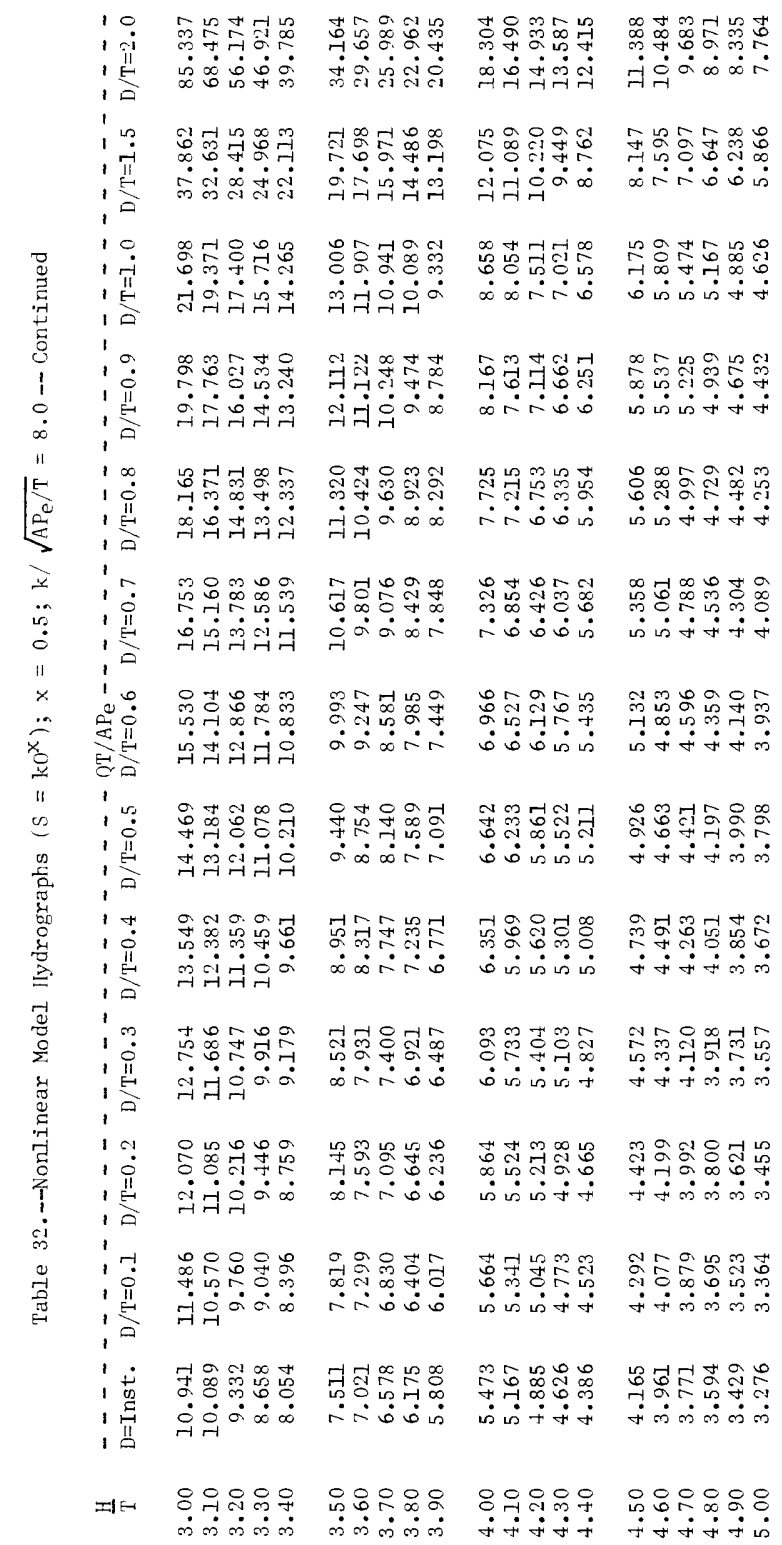




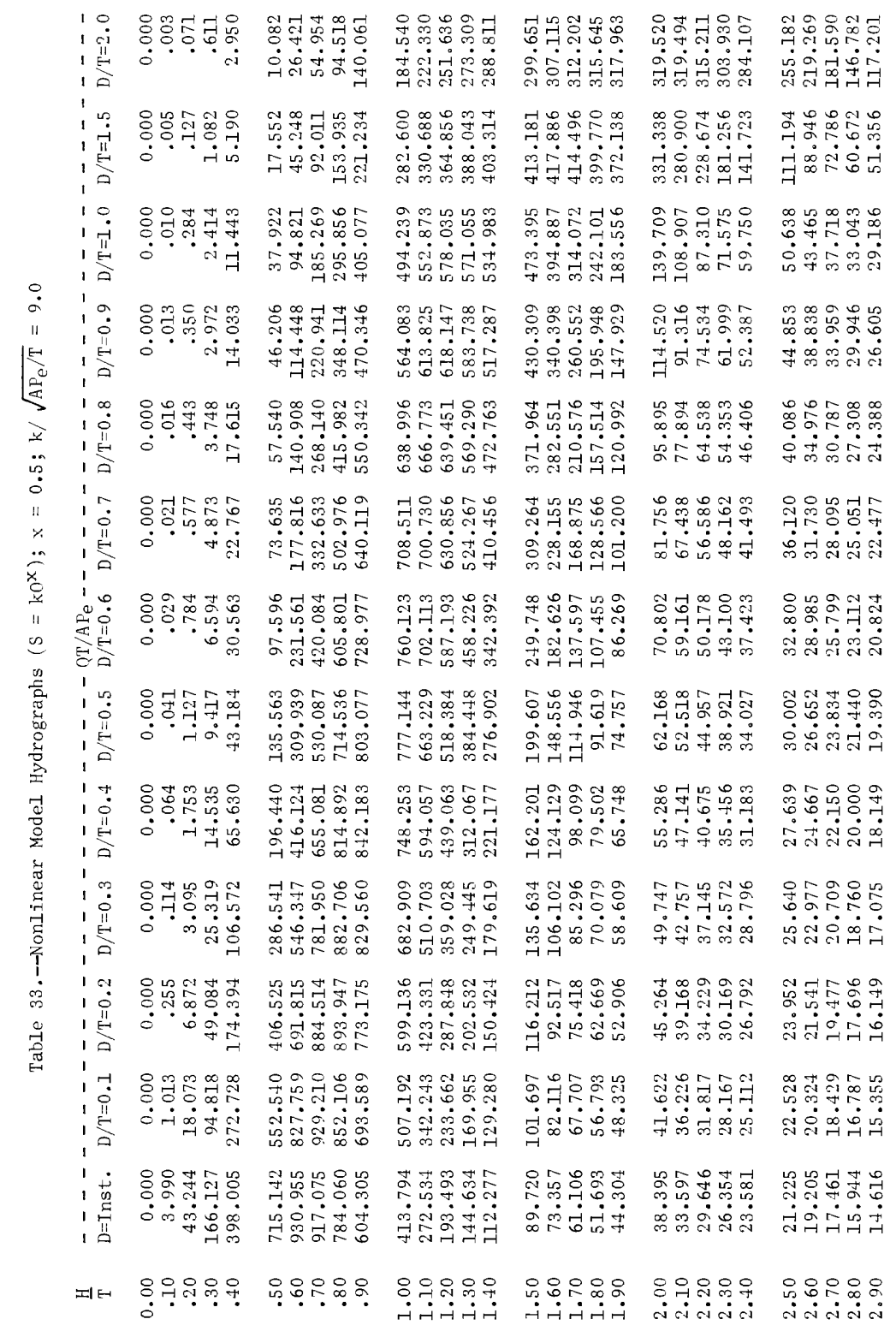




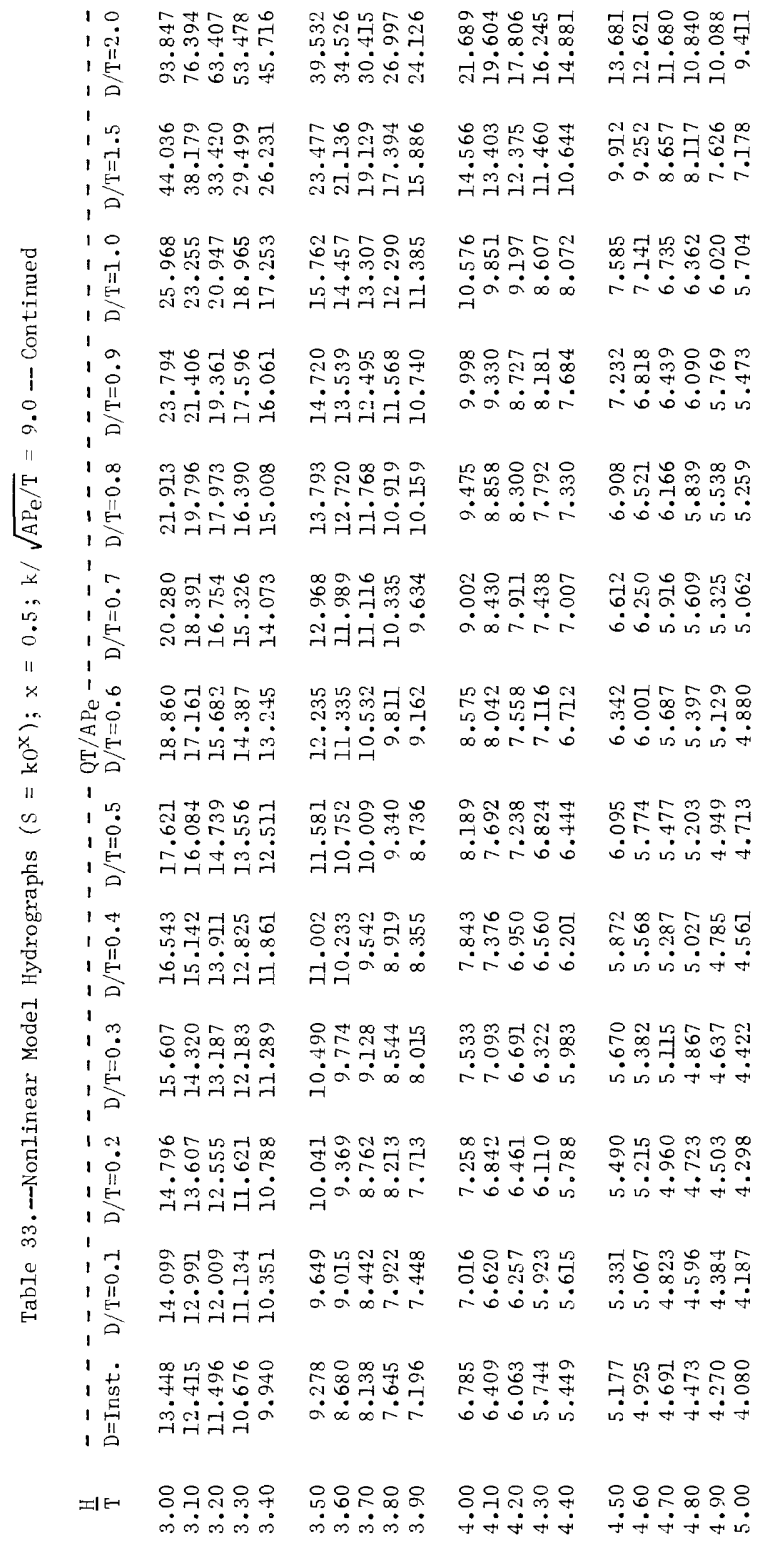




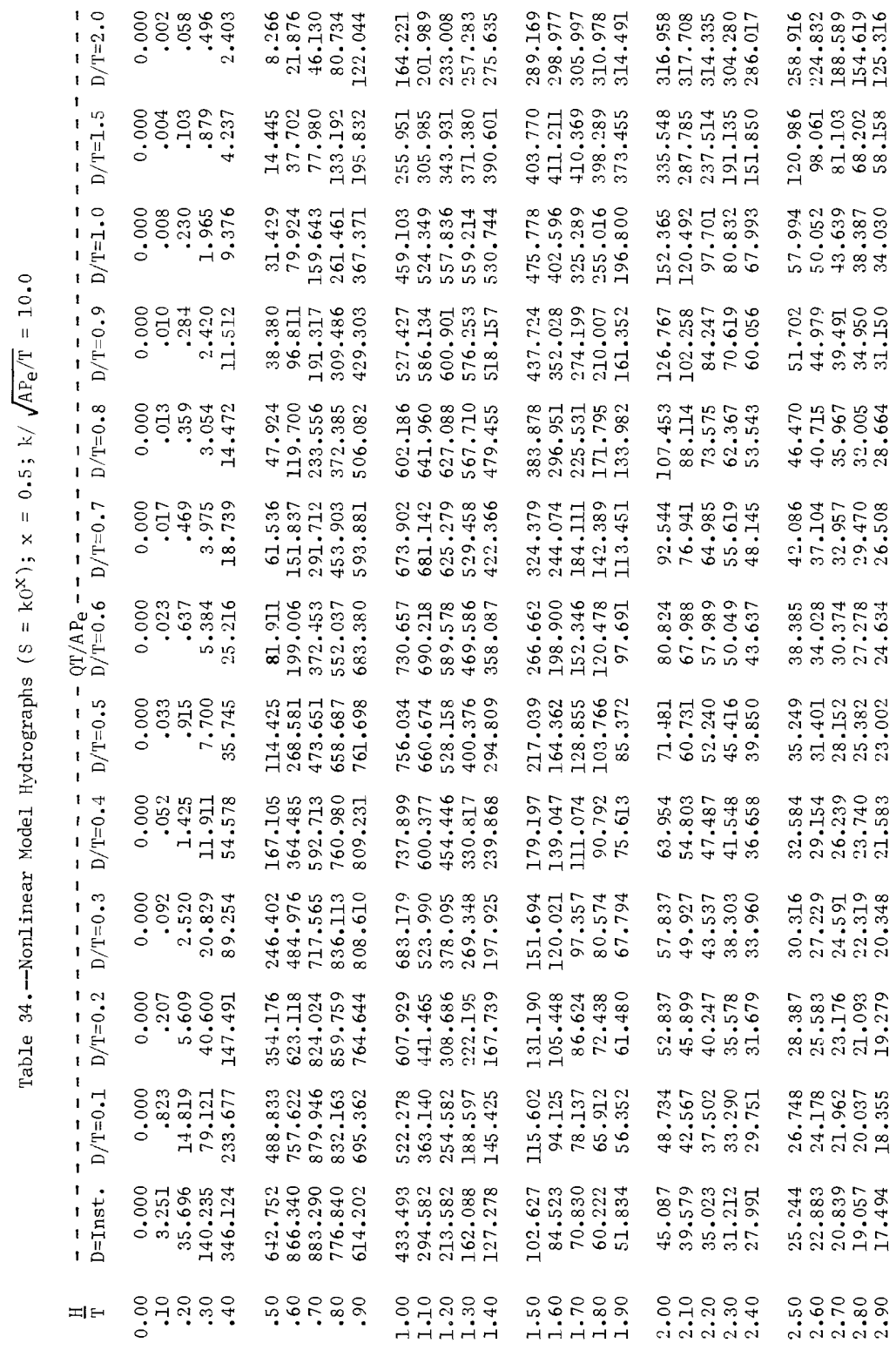




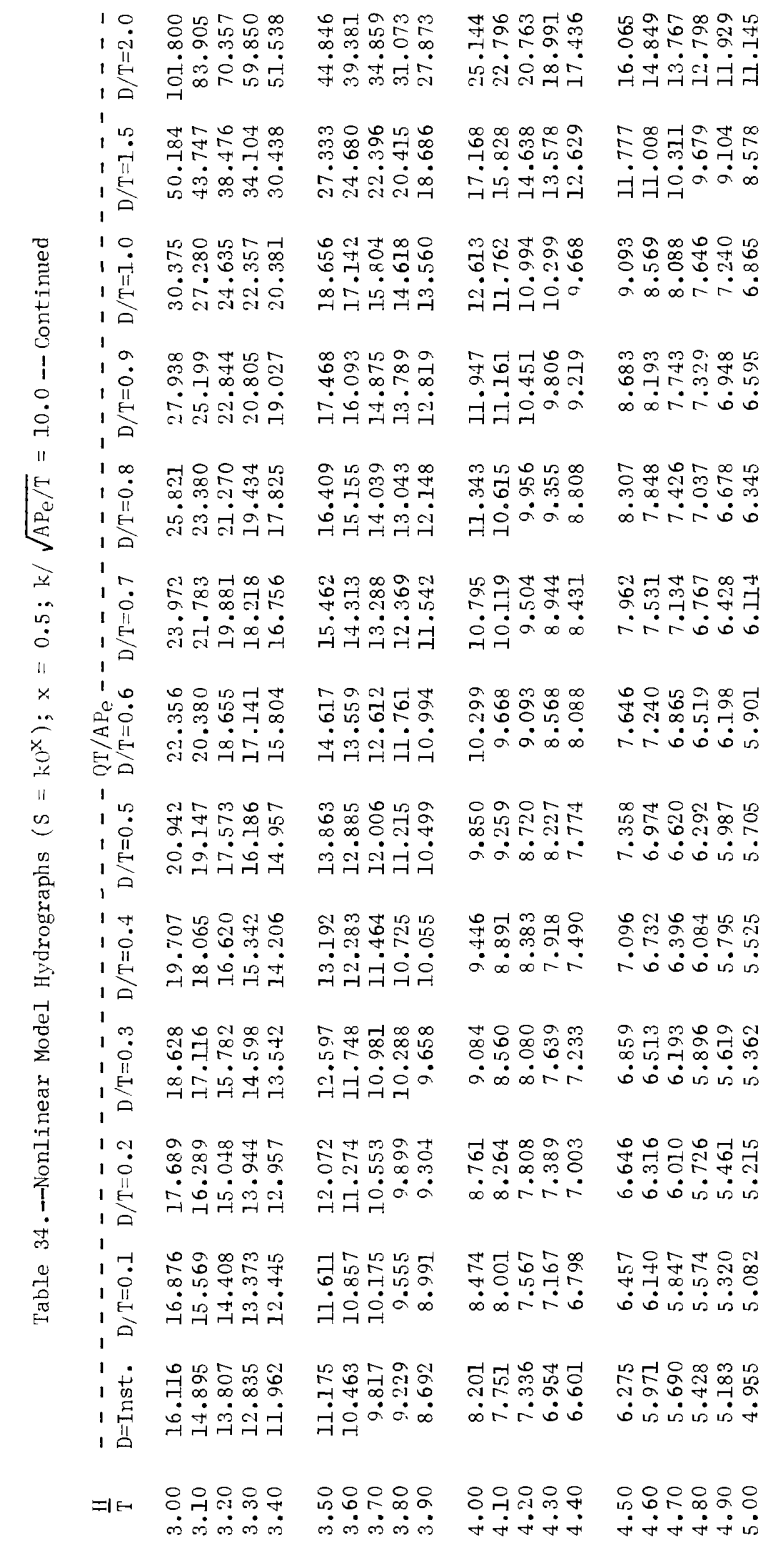




\begin{tabular}{|c|c|c|c|c|c|c|}
\hline $\begin{array}{l}10 \\
1 \stackrel{1}{1} \\
1 \% \\
10\end{array}$ & $\begin{array}{l}8 \\
8 \\
0\end{array}$ & 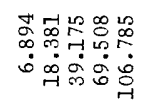 & 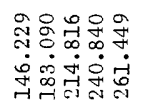 & 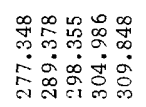 & 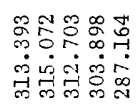 & 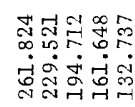 \\
\hline $\begin{array}{l}1 \\
1 \\
1 \\
110 \\
10\end{array}$ & 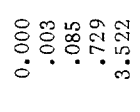 & 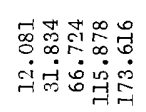 & 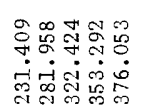 & 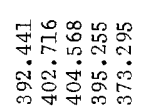 & 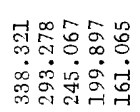 & 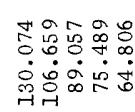 \\
\hline $\begin{array}{l}10 \\
1 \\
1 \\
1 \\
1.0\end{array}$ & 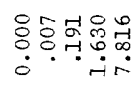 & 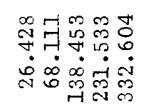 & 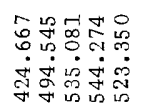 & 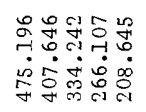 & 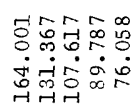 & 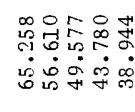 \\
\hline $\begin{array}{l}1 \\
1 \\
1 \\
1 \\
10 \\
10\end{array}$ & 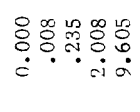 & 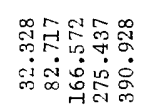 & 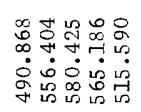 & 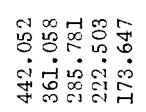 & 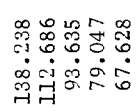 & 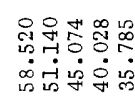 \\
\hline $\begin{array}{ll}1 & 0 \\
1 & 0 \\
1 & 11 \\
1 & 0\end{array}$ & 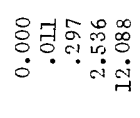 & 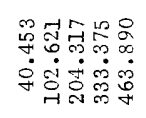 & 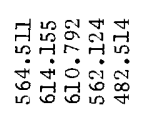 & 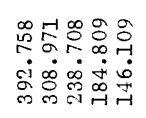 & 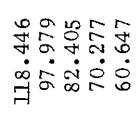 & 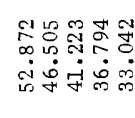 \\
\hline $\begin{array}{l}1 \\
10 \\
10 \\
0\end{array}$ & 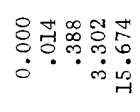 & 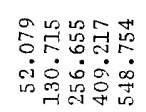 & 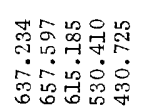 & 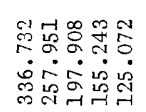 & 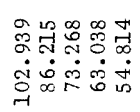 & 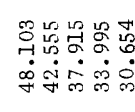 \\
\hline 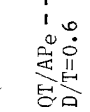 & $\begin{array}{l}0 \\
0 \\
0 \\
0 \\
0 \\
0 \\
0\end{array}$ & 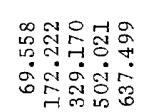 & 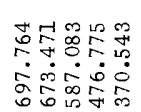 & 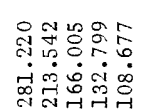 & 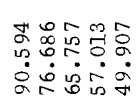 & 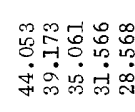 \\
\hline $\begin{array}{l}1 \\
1 \\
1 \\
1 \\
1 \\
1 \\
1 \\
10\end{array}$ & 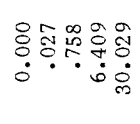 & 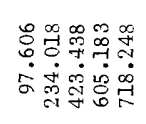 & 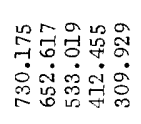 & 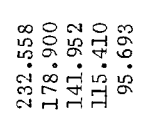 & 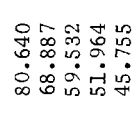 & 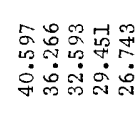 \\
\hline $\begin{array}{l}10 \\
1 \stackrel{0}{0} \\
1 \\
1 \\
0\end{array}$ & 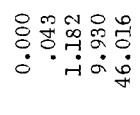 & 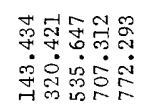 & 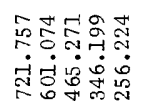 & 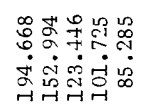 & 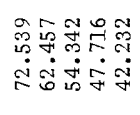 & 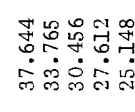 \\
\hline $\begin{array}{l}1 \\
1 \\
10 \\
10 \\
10\end{array}$ & 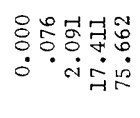 & 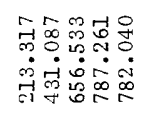 & 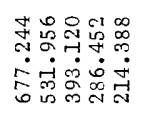 & 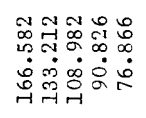 & 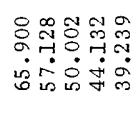 & 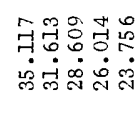 \\
\hline $\begin{array}{l}10 \\
10 \\
100 \\
100\end{array}$ & & 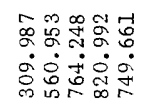 & 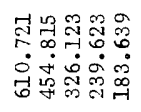 & 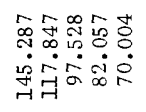 & 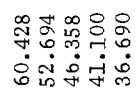 & 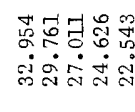 \\
\hline : & 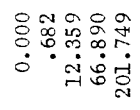 & 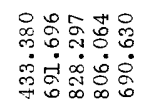 & 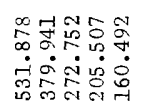 & 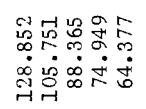 & 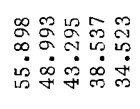 & 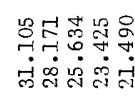 \\
\hline 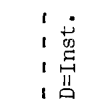 & 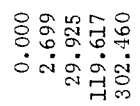 & 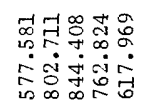 & 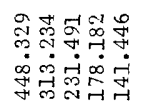 & 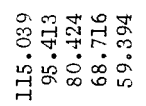 & 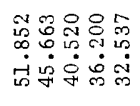 & 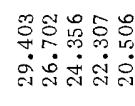 \\
\hline 푀타 & :윰윰요 & 웅ㅇㅇㅇㅇㅛ & 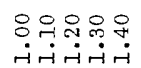 & 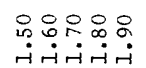 & 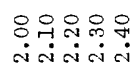 & \\
\hline
\end{tabular}




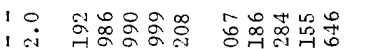

i il

i皆

is

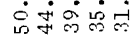

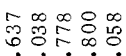

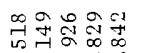

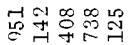

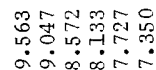

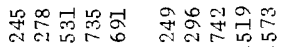

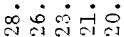

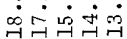

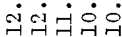

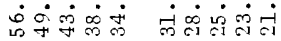

央융요용ㅇㅇ

수윰요

종웡요유

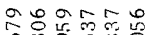

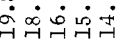

$\dot{9} \dot{\mathrm{G}} \dot{\mathrm{j}} \dot{\mathrm{j}}$

$\dot{0} \dot{\infty} \infty \dot{\infty} \infty$

$\therefore \therefore \dot{0} \dot{0}$

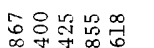

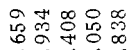

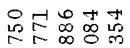

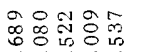

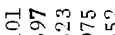

$0000 \infty$

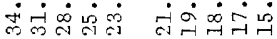

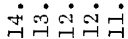

$\dot{0} \dot{0} \dot{0} \dot{\infty}$

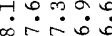

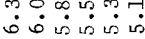

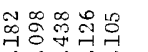

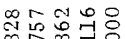

ํํㅇ요유ำ

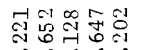

겅우유

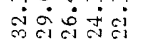

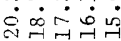

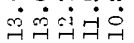

$\dot{\circ} \circ \circ \infty \infty$

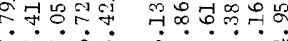

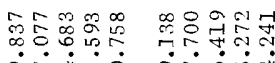

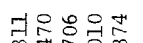

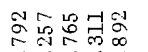

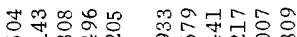

穴灾过完

मुं $\dot{7} \dot{-1} \dot{0}$

कंष्ठ

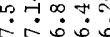

(1)

정응요유

선동요

a. 난유

도유유융

$\infty \infty \infty N$

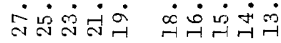

तi

व.

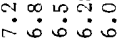

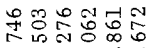

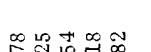

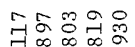

곡옹어웅

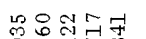

도요요

में

मํำ

تุنำ

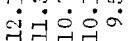

का

$\dot{0}$

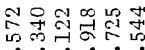

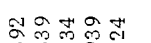

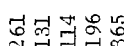

푸영요

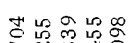

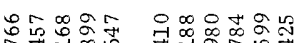

तं

$\dot{0}$ 近

붕ㅎㅇ.

$\dot{\infty} \infty \dot{0} \dot{\sim} \dot{\sim}$

$\dot{0} \dot{0} \dot{0}$ เं

मेंभुषं

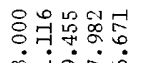

我起员

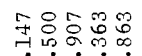

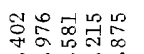

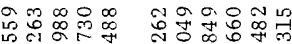

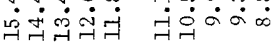

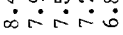

ด่

ம்

$\rightarrow$ mor

\section{की}

국ำ

กำ

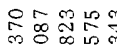

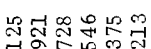

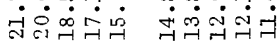

近 $\sigma^{\circ} \infty$

करำ

$\dot{0} \dot{0}$ เ

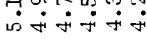

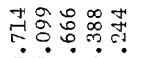

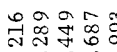

品䒘苦号品

$1+400$

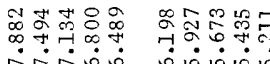

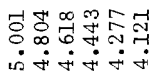

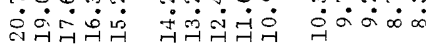

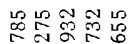

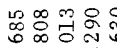

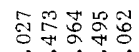

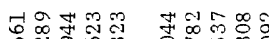

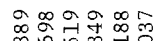

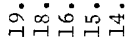

نें $\dot{\sim} \dot{\sim} \dot{-1}$

$\dot{0} \dot{\infty} \dot{\infty} \infty$

$\therefore \therefore \dot{0}$

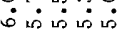

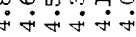

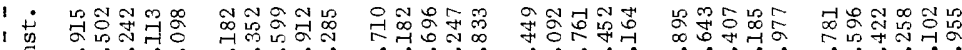

I

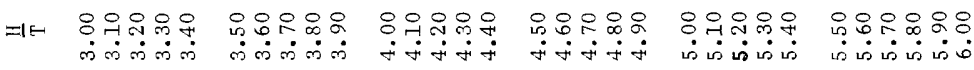




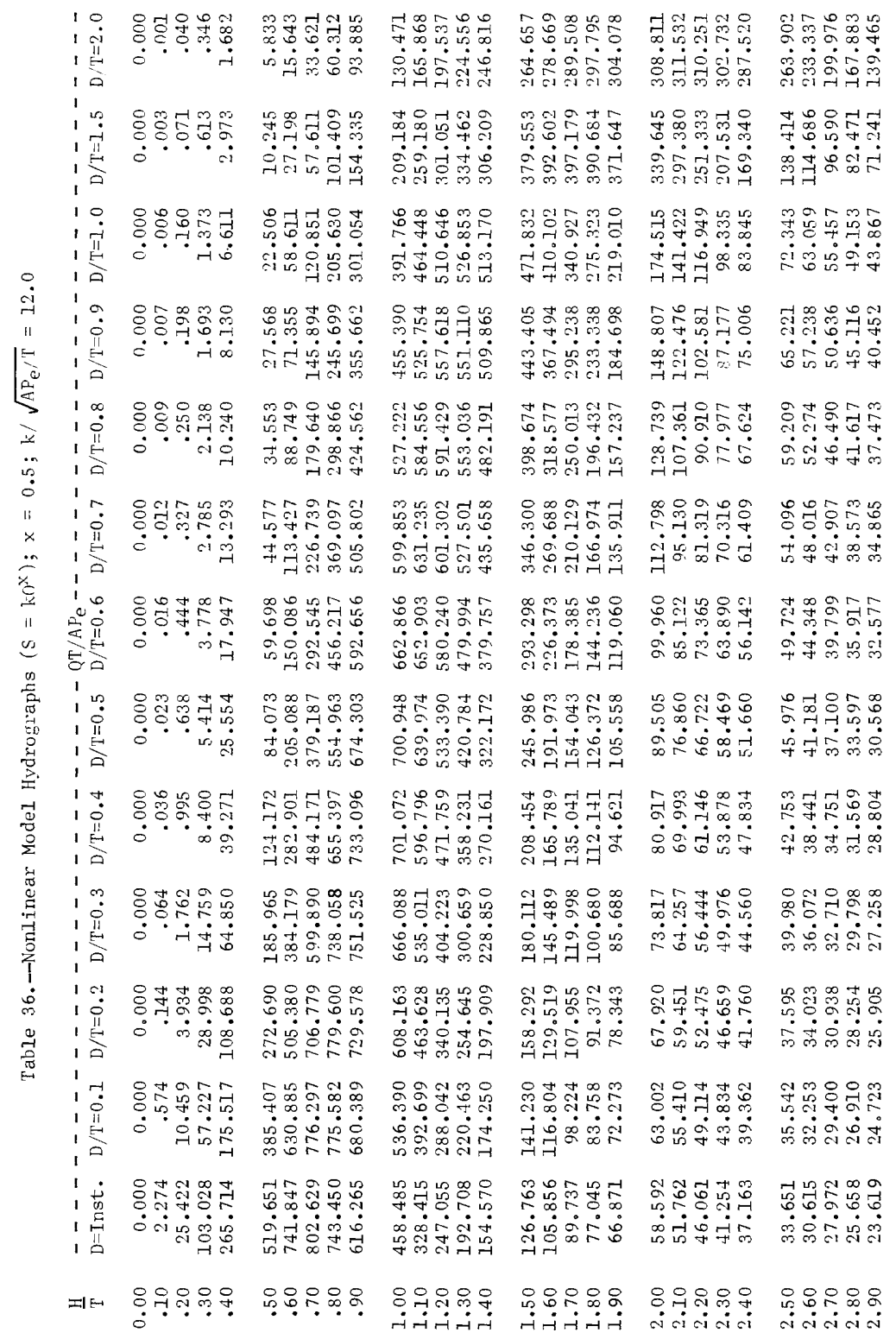




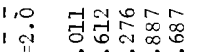

1 in

10

1

, II i⿱

10

1 击

19

$1 \overbrace{}^{1}$

$1 \infty$

i?

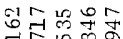

तुं

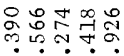

वें

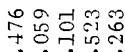

๑ळ

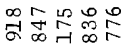

लिं

둥요요욤ㅇ

-

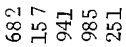

वंतेंत्तं

겅청요

ते

$\infty \% \infty$

눙ㅇㅇ

ㅇํㅇำำ-1

में $\dot{0} \dot{0}$

๓

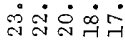

gotra

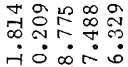

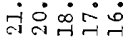

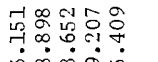

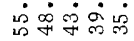

ํำำ.

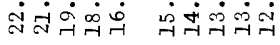

서에

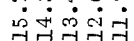

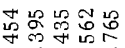

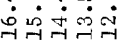

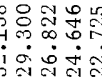

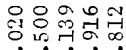

경요

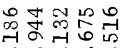

मं

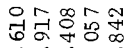

نं宀

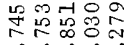

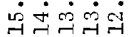

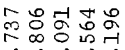

मेत

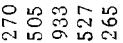

लेन्तन्त

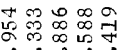

तiळ

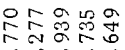

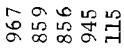

$\dot{0} \dot{0} \dot{0} \dot{0}$

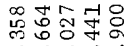

वi $\dot{7} \dot{-} \dot{0} \dot{\circ}$

क영문

$\dot{\omega} \dot{0} \dot{7} \dot{0}$

ભ

تं

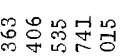

ज்

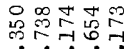

تं $\dot{0} \dot{0} 0$

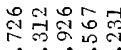

我离号?

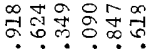

onNm

독ำ

봉요용

ஸ்

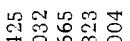

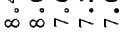

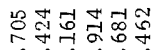

걱궉뎌

ซึ大

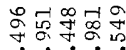

मुल्تं

$\dot{0} \therefore \dot{0} \dot{\infty} \infty$

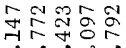

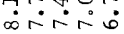

$\because \infty 0000$

$\dot{a} \rightarrow \dot{-1} \dot{0}$

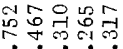

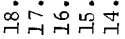

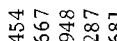

거ㅇㅝㅠ

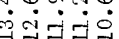

7070

$\therefore \approx 8 \infty$

年

공정용ㅇㅇ

귝ㅋㅋㅜ

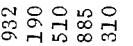

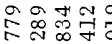

$\therefore \dot{\infty} \dot{\infty} \dot{\infty}$

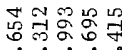

$\therefore \circ \dot{0}$

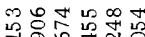
نे

$\infty$

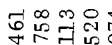

$\dot{7} \dot{7} \dot{7} \dot{0}$

में

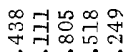

فำ

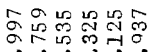

में

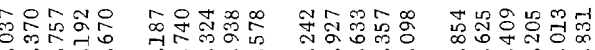
বن்

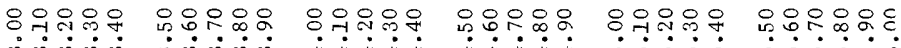

